\title{
Constituents from the Leaves of Tetraena mongolica and Their Protective Activity in HEK 293t Cells Damaged by $\mathrm{CdCl}_{2}$
}

Zhi-Gang Wu, Wei Wei, Hai-Yan Xu, Lin-Lin Zheng, Chao-Mei Ma*, and Ying-Chun Wang*

College of Life Sciences, Inner Mongolia University, Huhhot, Inner Mongolia, China

* Corresponding author: Yingchun Wang, Fax: +86-0471-4992442.

E-mail address: ycwang@imu.edu.cn

Chaomei Ma, Fax: +86-0471- 4992435.

E-mail address: $\underline{\text { cmma@imu.edu.cn }}$ 


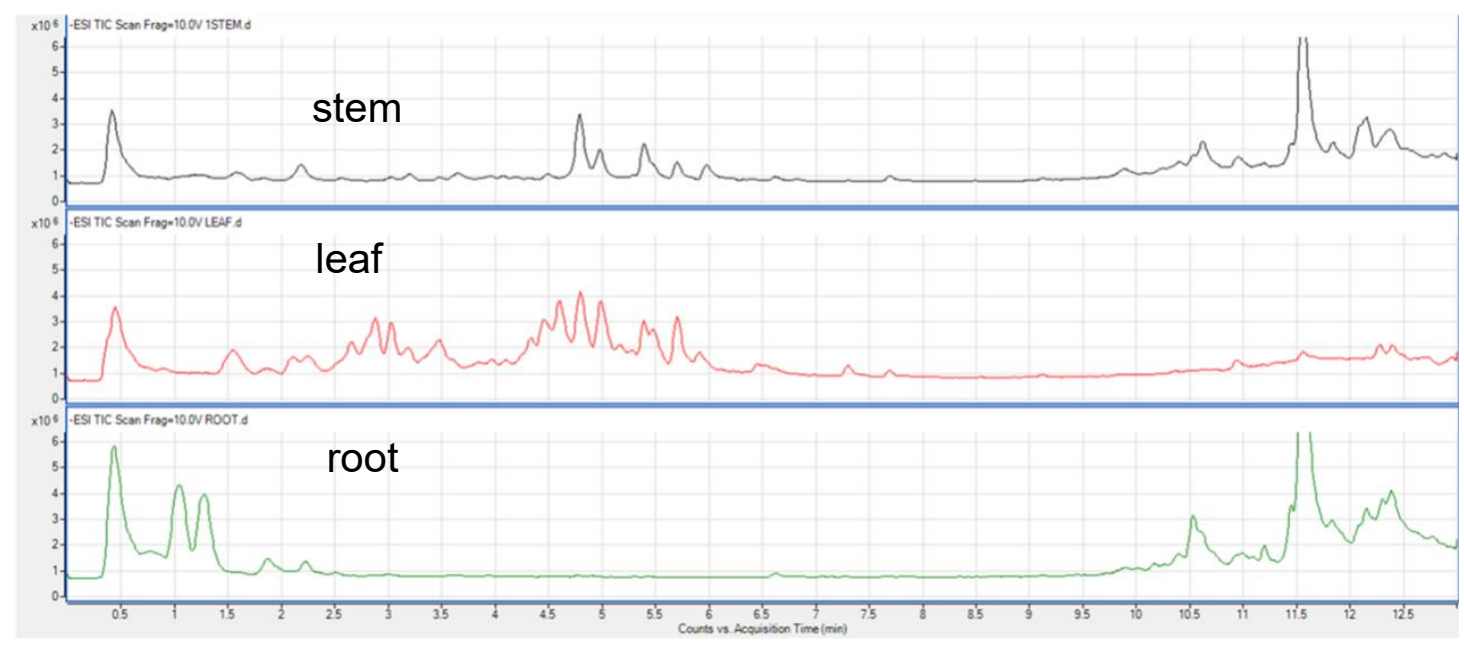

Figure S1. UHPLC-MS analysis of the extracts of different parts of Tetraena<smiles>[R]OCc1ccccc1COC(C)=O</smiles><smiles>[R2]Oc1c(-c2ccc(O)c(O)c2)oc2cc(O)cc(O)c2c1=O</smiles><smiles>[R9]Oc1c(-c2ccc(O)c(O)c2)oc2cc(O)cc(O)c2c1=O</smiles><smiles>COc1cc(-c2oc3cc(O)cc(O)c3c(=O)c2O[123I])ccc1O</smiles>

13


Figure S2. Structures of known compounds isolated from T. mongolica. 
Compound 7: amorphous solid, UPLC-MS $m / z 385[\mathrm{M}-\mathrm{H}]^{-},{ }^{1} \mathrm{H}$ NMR (DMSO- $d_{6}$ $500 \mathrm{MHz}$ ) data, $\delta_{\mathrm{H}}: 2.41$ (d, J=16.8 Hz, H-2a), 2.04 (d, J=16.8 Hz, H-2b), 5.77 (overlap, H-4), 5.77 (overlap, H-7), 5.77 (overlap, H-7), 4.32 (quit, J=6.4 Hz, H-9), 1.17 (d, $J=6.4 \mathrm{~Hz}, \mathrm{H}-10), 0.92$ (s, H-11, H-12), 1.80 (d, J=1.4 Hz, H-13), 4.16 (d, J=7.8 Hz, H-1'), 2.92 (overlap, H-2'), 3.10 (overlap, H-3'), 3.02 (overlap, H-4'), 3.02 (overlap, H-5'), 3.62 (m, H-6'a), 3.41 (m, H-6’b). cc: $^{\prime} 41.35$ (C-1), 49.77 (C-2), 197.77 (C-3), 126.10 (C-4), 164.38 (C-5), 78.27 (C-6), 130.75 (C-7), 133.73 (C-8), 75.25 (C-9), 21.30 (C-10), 24.49 (C-11), 23.46 (C-12), 19.36 (C-13), 101.30 (C-1'), 74.07 (C-2'), $77.26\left(\mathrm{C}-3^{\prime}\right), 70.46\left(\mathrm{C}-4^{\prime}\right), 77.26\left(\mathrm{C}-5^{\prime}\right), 61.52\left(\mathrm{C}-6^{\prime}\right)$.

Compound 8: amorphous solid, UPLC-MS $m / z 269[\mathrm{M}-\mathrm{H}]^{-},{ }^{1} \mathrm{H}$ NMR (DMSO- $d_{6}$ $500 \mathrm{MHz})$ data, $\delta_{\mathrm{H}}: 7.38$ (m, H-1, H-5), 7.30 (m, H-2, H-4), 7.27 (m, H-3), 4.82 (d, $J=12.2 \mathrm{~Hz}, \mathrm{H}-6 \mathrm{a}), 4.57$ (d, J=12.2 Hz, H-6b), 4.22 (d, J=7.8 Hz, H-1'), 3.00 (m, H-2'), 3.14 (m, H-3'), 3.06 (m, H-4'), 3.07 (m, H-5'), 3.46 (m, H-6a'), 3.69 (m, H-6b'). $\delta_{C}$ : 128.05 (C-1, C-5), 128.56 (C-2, C-4), 127.77 (C-3), 138.52 (C-6), 102.53 (C-1'), 70.58 (C-2'), $73.96\left(\mathrm{C}-3^{\prime}\right), 77.2\left(\mathrm{C}-4^{\prime}\right), 77.3\left(\mathrm{C}-5^{\prime}\right), 61.6\left(\mathrm{C}-6^{\prime}\right)$

Compound 9: amorphous solid, UPLC-MS m/z 297 [M-H] ${ }^{-},{ }^{1} \mathrm{H}$ NMR (DMSO- $d_{6}$ $500 \mathrm{MHz}$ ) data, $\delta_{\mathrm{H}}: 7.1$ (d, J=8.8 Hz, H-1, H-5), 7.9 (d, J=8.8 Hz, H-2, H-4), 2.52 (s, H-Me), 5.0 (d, J=7.3 Hz, H-1'), 3.25 (m, H-2'), 3.26 (m, H-3'), 3.16 (m, H-4'), 3.36 (m, H-5'), 3.46 (m, H-6a'), 3.67 (m, H-6b'). $\delta_{\mathrm{c}}: 116.28$ (C-1, C-5), 130.71 (C-2, C-4), 131.26 (C-3), 161.51 (C-6), 196.86 (C-7), 100.21 (C-1'), 73.60 (C-2'), 76.00 (C-3'), $70.00\left(\mathrm{C}-4^{\prime}\right), 77.59\left(\mathrm{C}-5^{\prime}\right), 61.06\left(\mathrm{C}-6^{\prime}\right), 26.93(\mathrm{Me})$.

Compound 10: amorphous solid, UPLC-MS m/z 327 [M-H] $]^{-},{ }^{1} \mathrm{H}$ NMR (DMSO- $d_{6}$ 
$500 \mathrm{MHz}$ ) data, $\delta_{\mathrm{H}}: 7.46$ (d, J=2 Hz, H-2), 7.56 (dd, $\left.J=8.5,2 \mathrm{~Hz}, \mathrm{H}-4\right), 7.17$ (d, J=8.5 Hz, H-5), 2.52 (s, H-Me), 3.82 (s, H-OMe), 5.0 (d, J=7.3 Hz, H-1'), 3.25 (m, H-2'), 3.26 (m, H-3'), 3.16 (m, H-4'), 3.36 (m, H-5'), 3.46 (m, H-6a'), 3.67 (m, H-6b'). $\delta_{\mathrm{C}}$ : 149.11 (C-1), 111.38 (C-2), 131.24 (C-3), 123.12 (C-4), 114.57 (C-5), 151.07 (C-6), 26.90 (C-Me), 56.05 (C-OMe).

Compound 15: yellow powder, UPLC-MS m/z $623[\mathrm{M}-\mathrm{H}]^{-},{ }^{1} \mathrm{H}$ NMR (DMSO- $d_{6} 500$ MHz) data, $\delta_{\mathrm{H}}: 6.13$ (s, H-6), 6.35 (s, H-8), 7.83 (s, H-2'), 6.89 (d, J=8.4 Hz, H-5'), 7.49 (d, J=8.4 Hz, H-6'), 5.40 (d, J=7.3 Hz, H-1' '), 3.00-3.7 (11H, m), 4.40 (s, H-1' ' ), 3.81 (s, H-OMe), 0.96 (d, J=6.2 Hz, H-6"'). $\delta_{\mathrm{C}}: 156.64$ (C-2, C-9), 133.36 (C-3), 177.51 (C-4), 161.55(C-5), 99.57 (C-6), 166.08 (C-7), 94.43 (C-8), 103.90 (C-10), $122.64\left(\mathrm{C}-1^{\prime}\right), 115.67\left(\mathrm{C}-2^{\prime}\right), 147.32\left(\mathrm{C}-3^{\prime}\right), 149.88\left(\mathrm{C}-4^{\prime}\right), 121.46\left(\mathrm{C}-5^{\prime}\right), 101.73(\mathrm{C}-$ $\left.1^{\prime \prime}\right), 74.72\left(\mathrm{C}-2^{\prime \prime}\right), 76.33\left(\mathrm{C}-3^{\prime \prime}\right), 70.73$ (C-4"'), 76.83 (C-5'), 67.27 (C-6"), 101.32 $\left(\mathrm{C}-1^{\prime \prime \prime}\right), 70.50\left(\mathrm{C}-2^{\prime \prime \prime}\right), 70.02\left(\mathrm{C}-3^{\prime \prime \prime}\right), 72.22\left(\mathrm{C}-4^{\prime \prime \prime}\right), 68.72\left(\mathrm{C}-5^{\prime \prime \prime}\right), 18.15\left(\mathrm{C}-6^{\prime \prime \prime}\right)$, 56.07 (C-OMe).

Compounds 11-14: the authentic were purchased CHENGDU MUST BIOTECHNOLOGY CO.,LTD. The column was an Agilent SB-C ${ }_{18}$ (RHD $1.8 \mu \mathrm{m}, 2.1 \times 100$ $\mathrm{mm}$ ), the mobile phase was $\mathrm{H}_{2} \mathrm{O}(\mathrm{A})$ and $\mathrm{MeOH}(\mathrm{B}), 0 \mathrm{~min}, 25 \% \mathrm{~B} ; 5.5 \mathrm{~min}, 60 \% \mathrm{~B} ; 7$ min, 100\% B, 9 min, 100\% B. 1290 UPLC and an Agilent 6430 triple quadrupole MS system, 




Figure S3.The ${ }^{1} \mathrm{H}$ NMR spectrum of compound 1 in DMSO-d6 $(500 \mathrm{~Hz})$

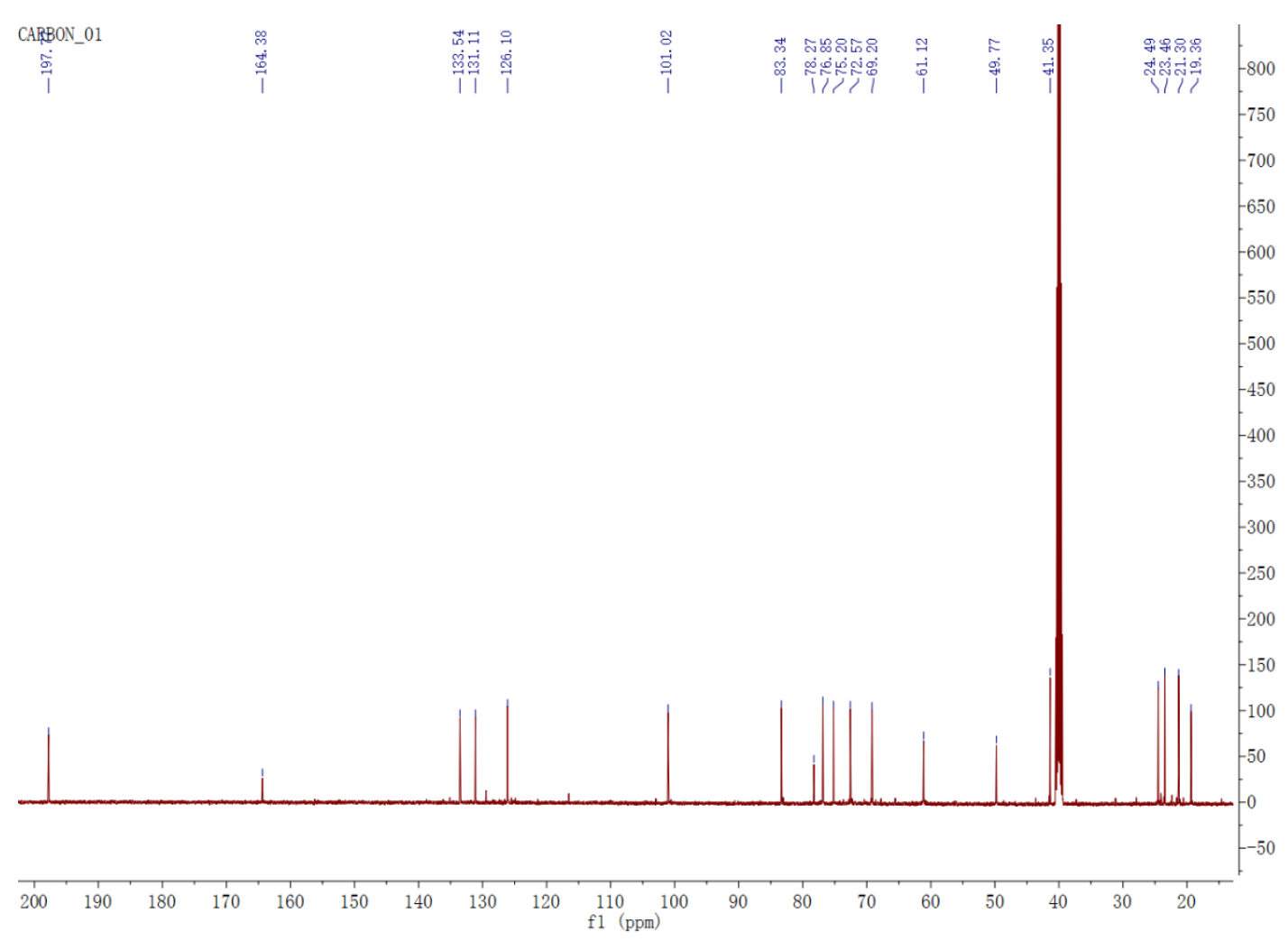

Figure S4. The ${ }^{13} \mathrm{C}$ NMR spectrum of compound 1 in DMSO-d6 $(500 \mathrm{~Hz})$ 




Figure S5. The HSQC spectrum of compound 1 in DMSO-d6 $(500 \mathrm{~Hz})$



Figure S6. The HMBC spectrum of compound 1 in DMSO-d6 $(500 \mathrm{~Hz})$ 


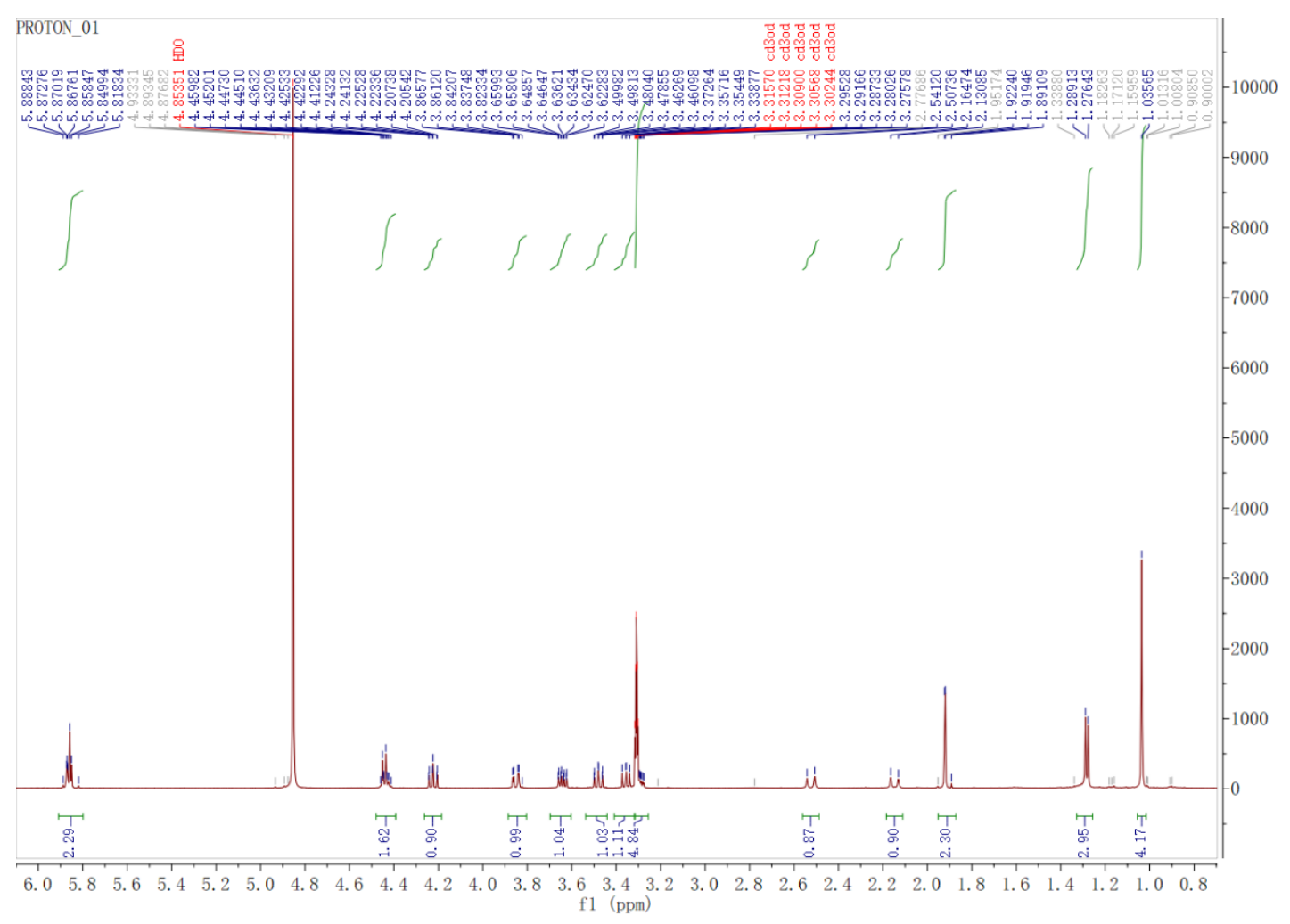

Figure S7. The ${ }^{1} \mathrm{H}$ NMR spectrum of compound 1 in $\mathrm{CDOD}_{3}(500 \mathrm{~Hz})$



Figure S8. The ${ }^{13} \mathrm{C}$ NMR spectrum of compound 1 in $\mathrm{CDOD}_{3}(500 \mathrm{~Hz})$ 


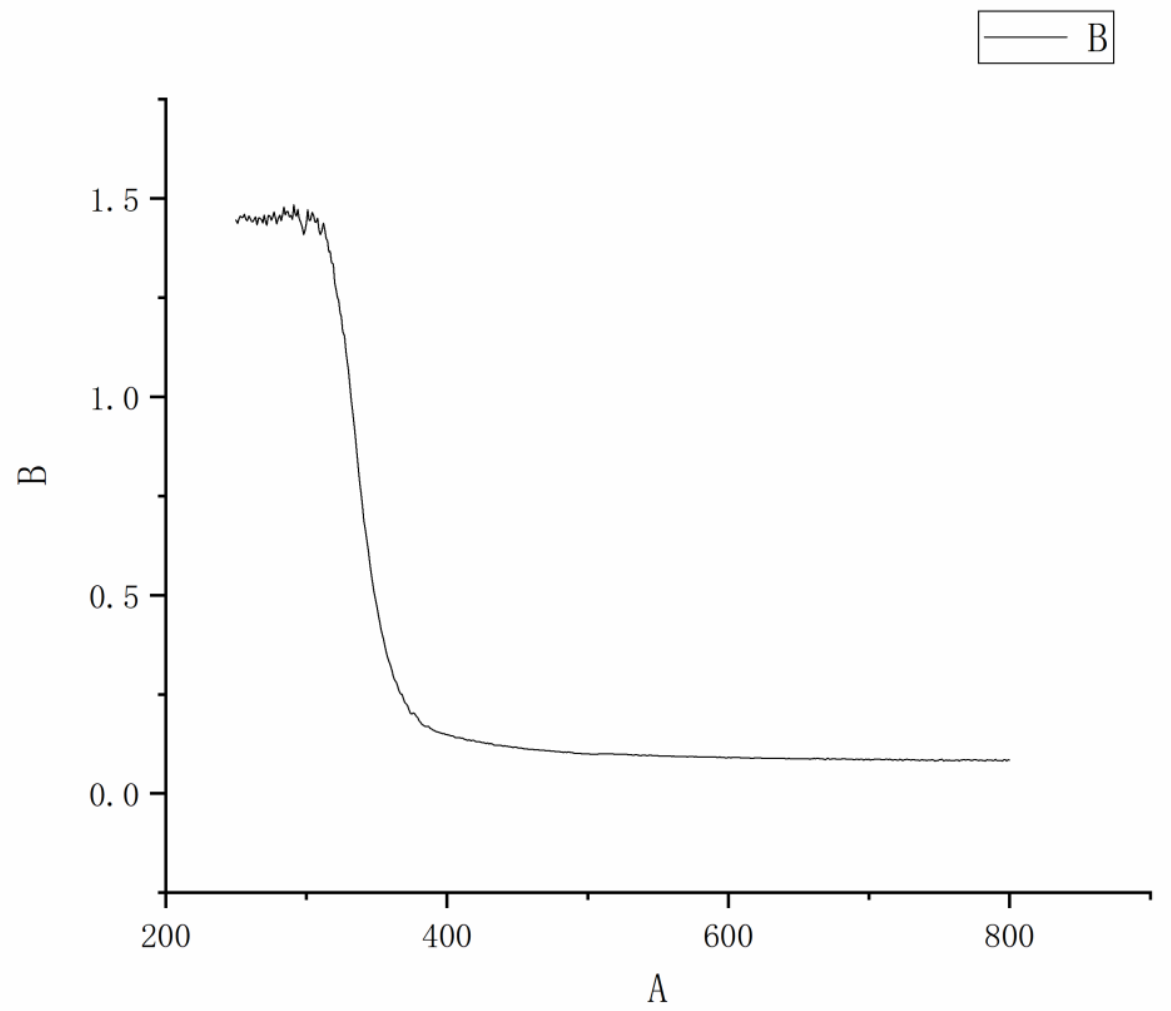

Figure S9. The UV spectra of compound 1.

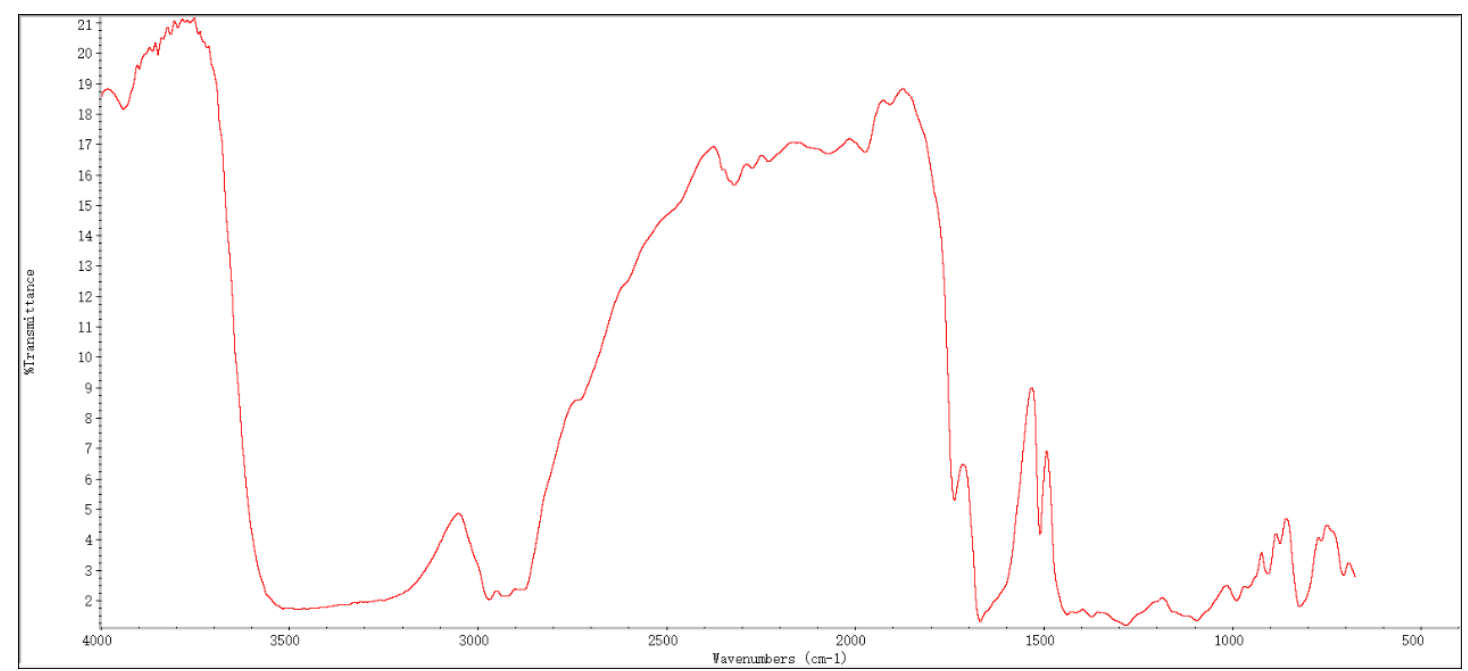

Figure S10. The IR spectra of compound 1 


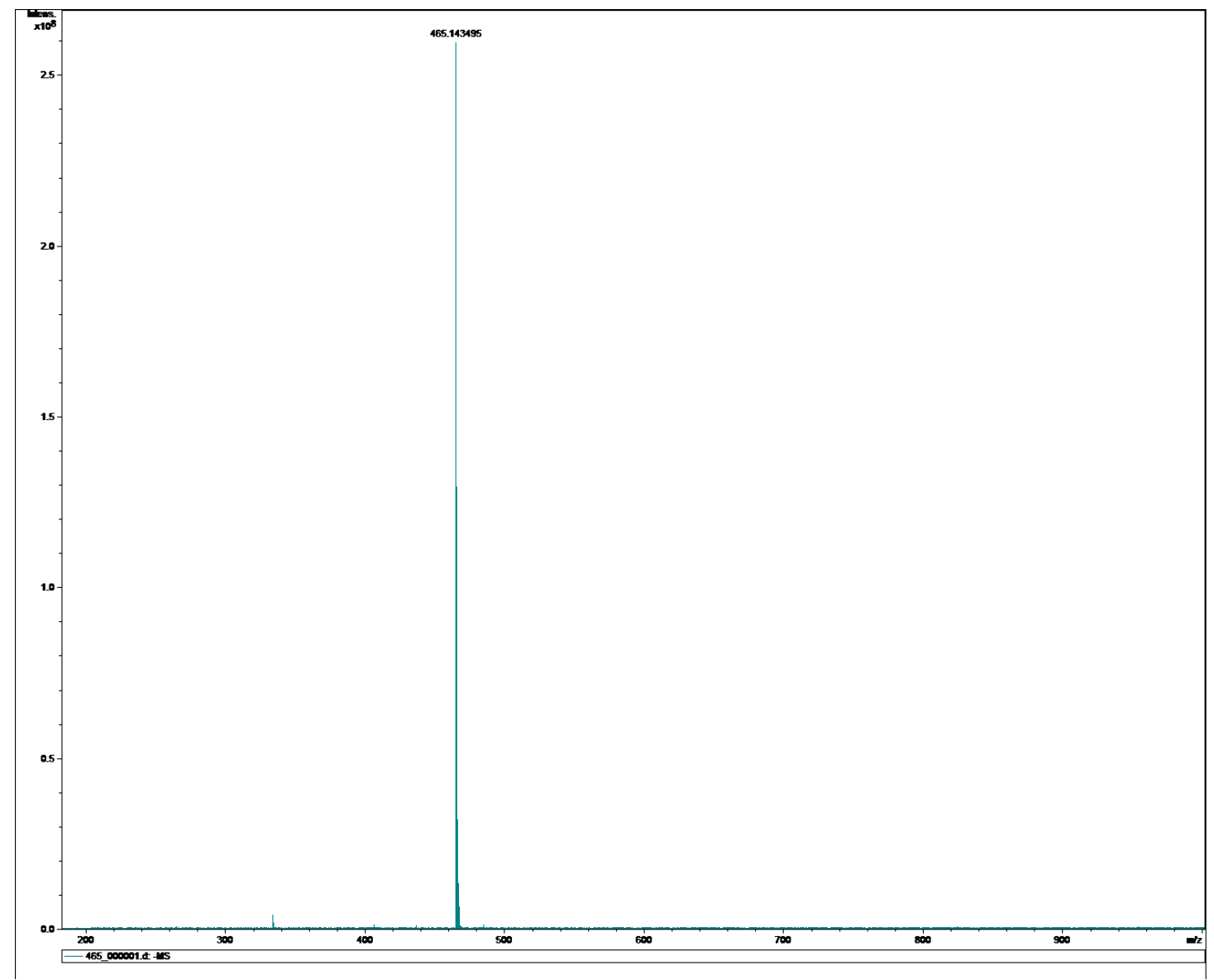

Figure S11. The ESI-TOF-MS spectra of compound 1

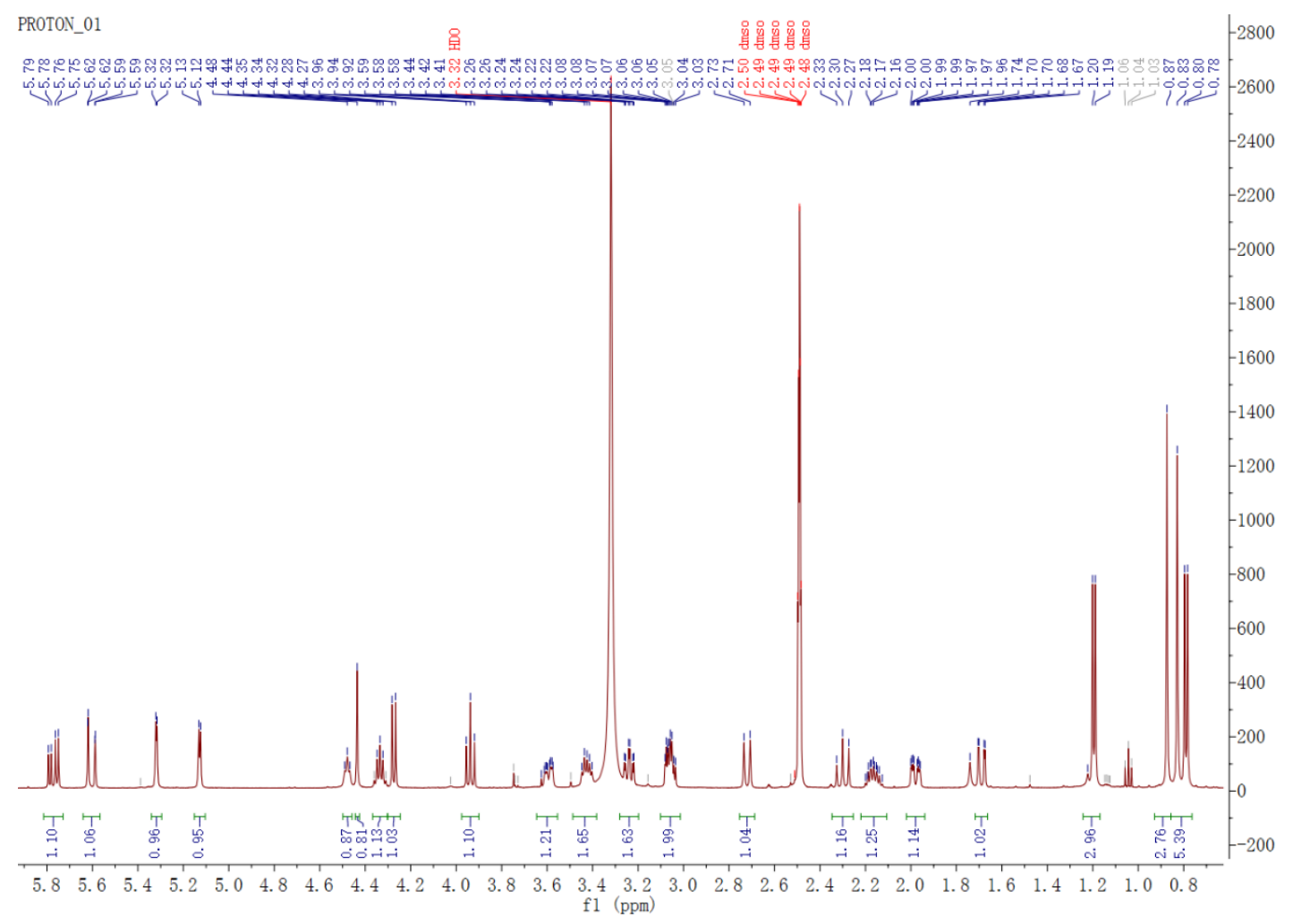

Figure S12. The ${ }^{1} \mathrm{H}$ NMR spectrum of compound 2 in DMSO-d6 $(500 \mathrm{~Hz})$ 


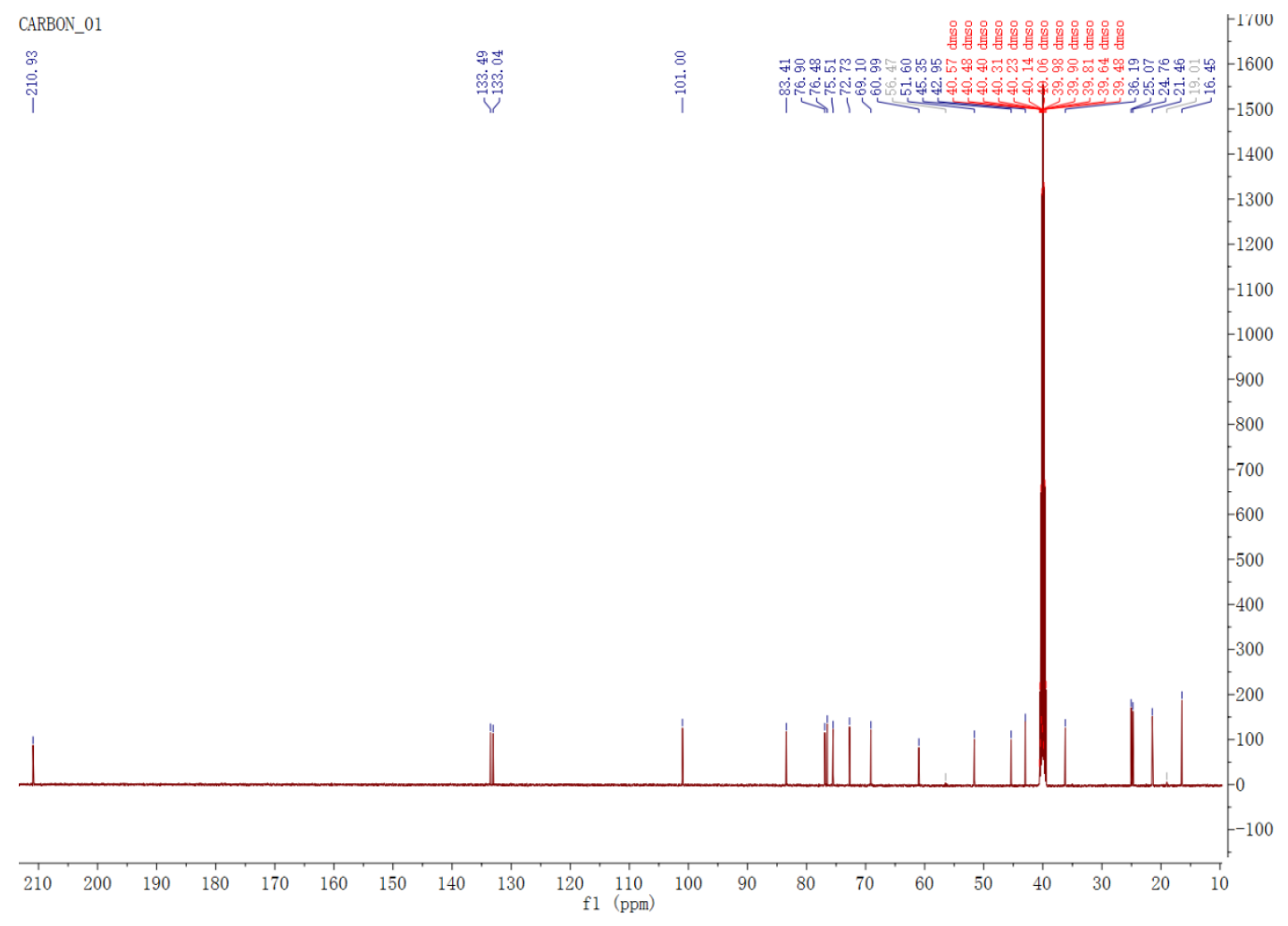

Figure S13. The ${ }^{13} \mathrm{C}$ NMR spectrum of compound 2 in DMSO-d6 $(500 \mathrm{~Hz})$ 


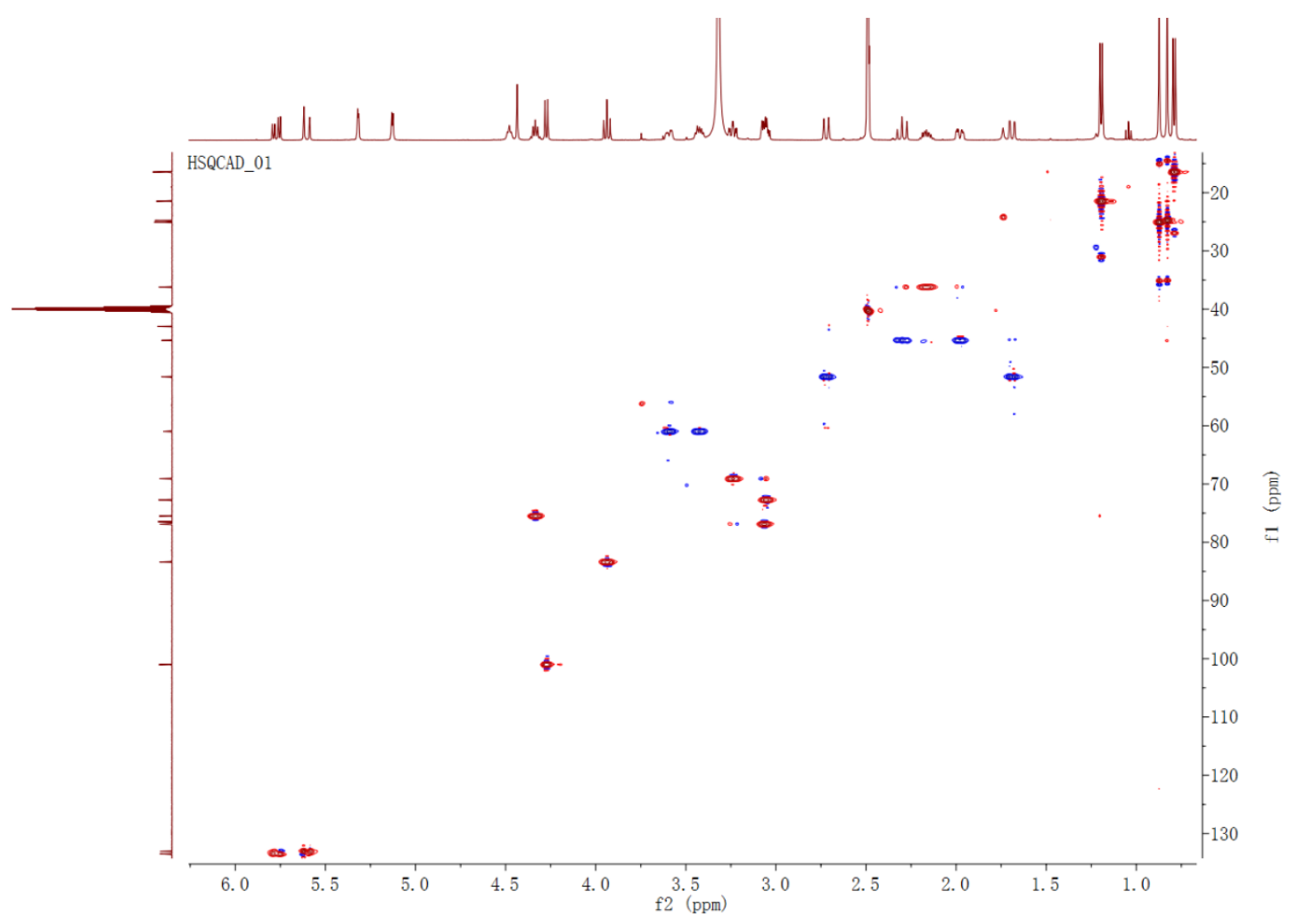

Figure S14. The HSQC spectrum of compound 2 in DMSO-d6 $(500 \mathrm{~Hz})$

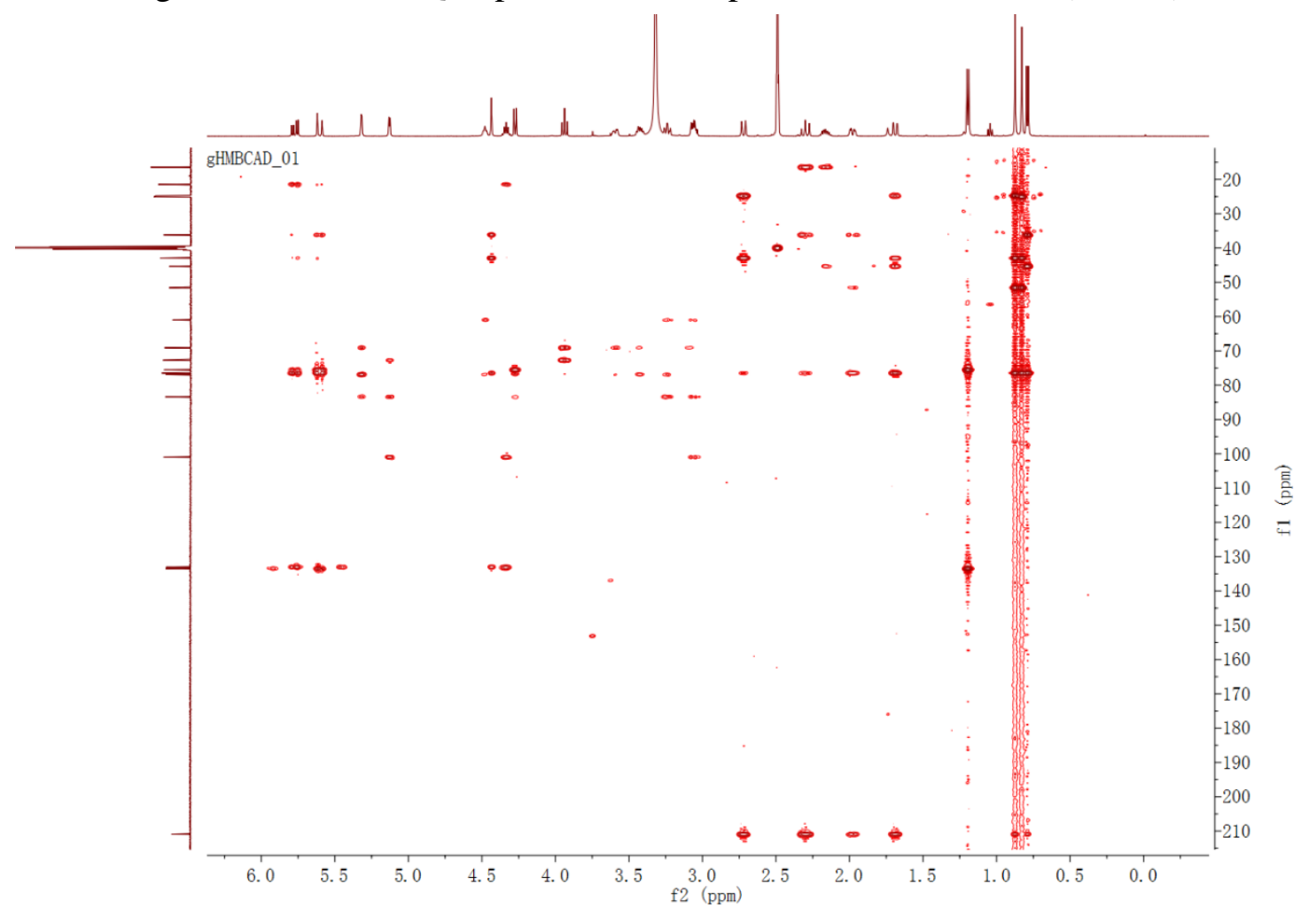

Figure S15. The HMBC spectrum of compound 2 in DMSO-d6 $(500 \mathrm{~Hz})$ 


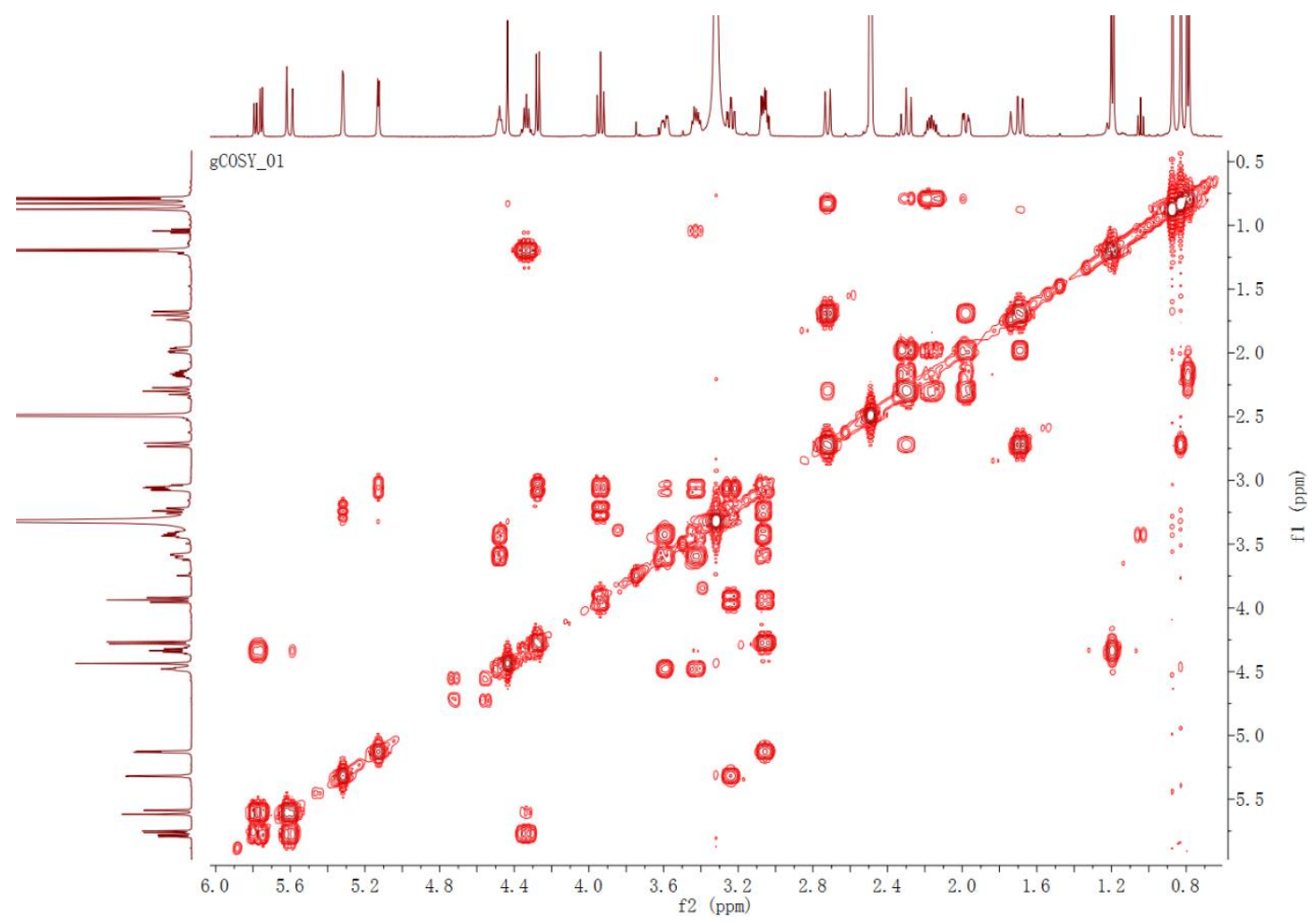

Figure S16. The ${ }^{1} \mathrm{H}-{ }^{1} \mathrm{H}$ COSY spectrum of compound 2 in DMSO- $d 6(500 \mathrm{~Hz})$

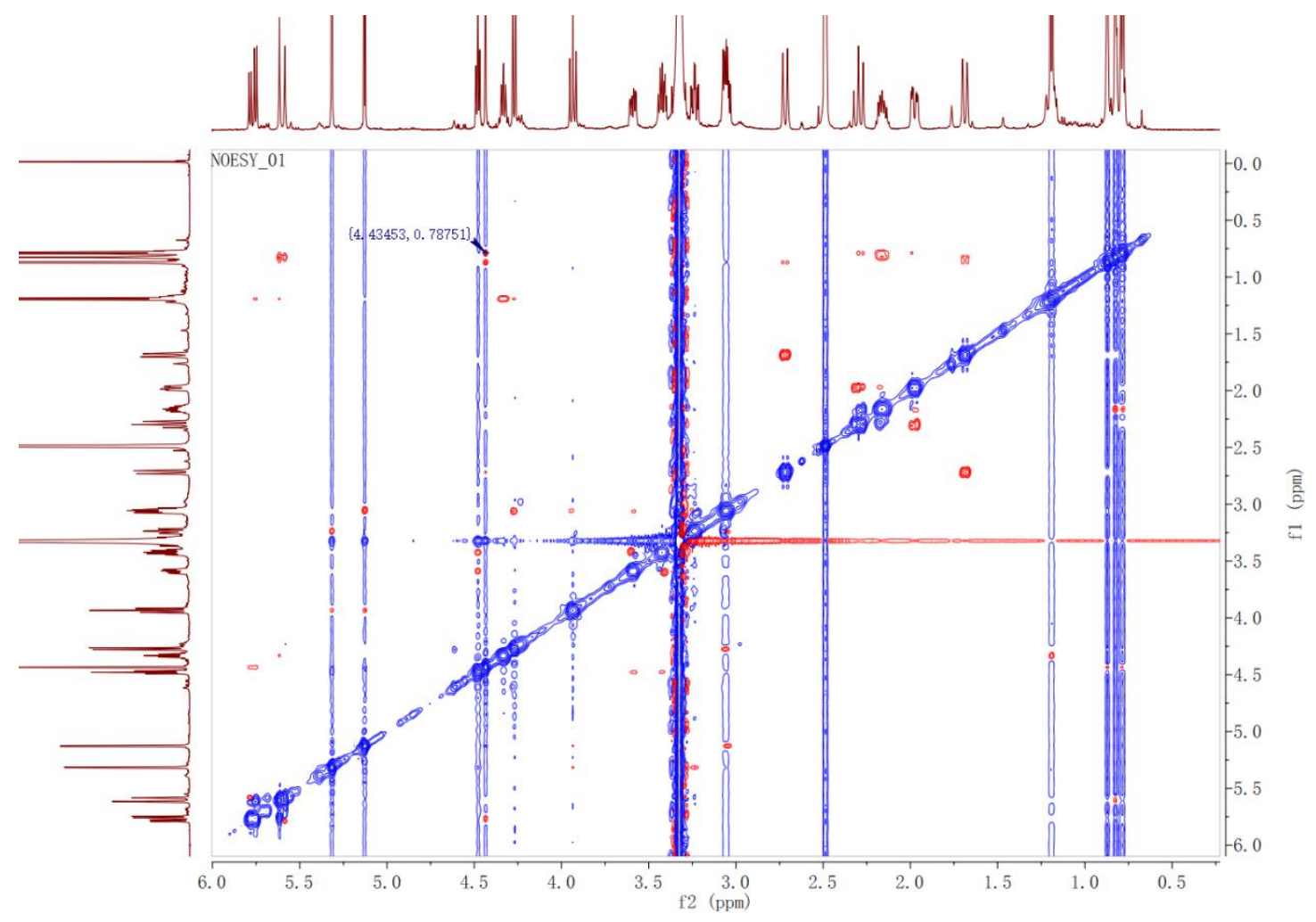

Figure S17. The NOESY spectrum of compound 2 in DMSO-d6 $(500 \mathrm{~Hz})$ 


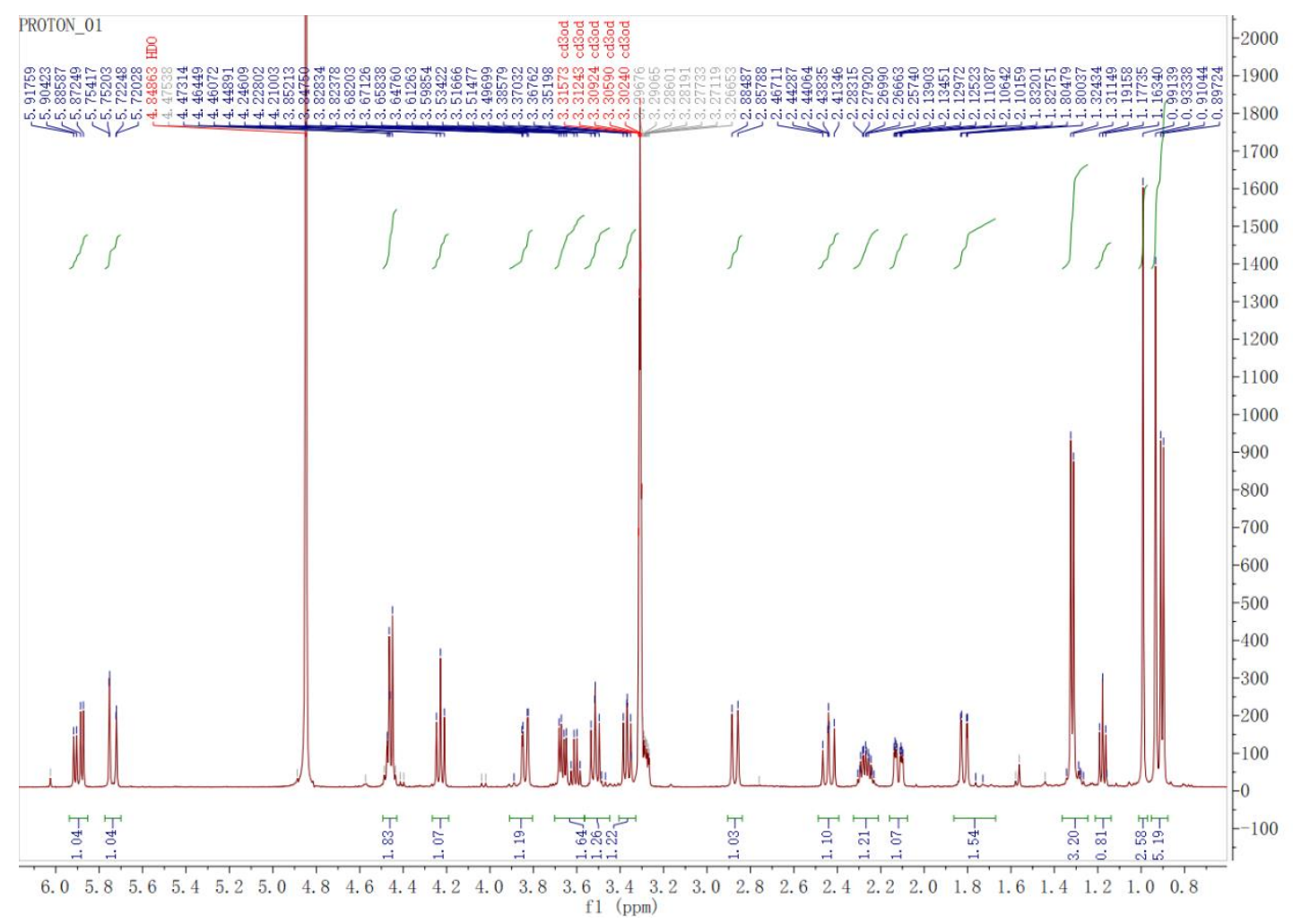

Figure S18. The ${ }^{1} \mathrm{H}$ NMR spectrum of compound 2 in $\mathrm{CDOD}_{3}(500 \mathrm{~Hz})$

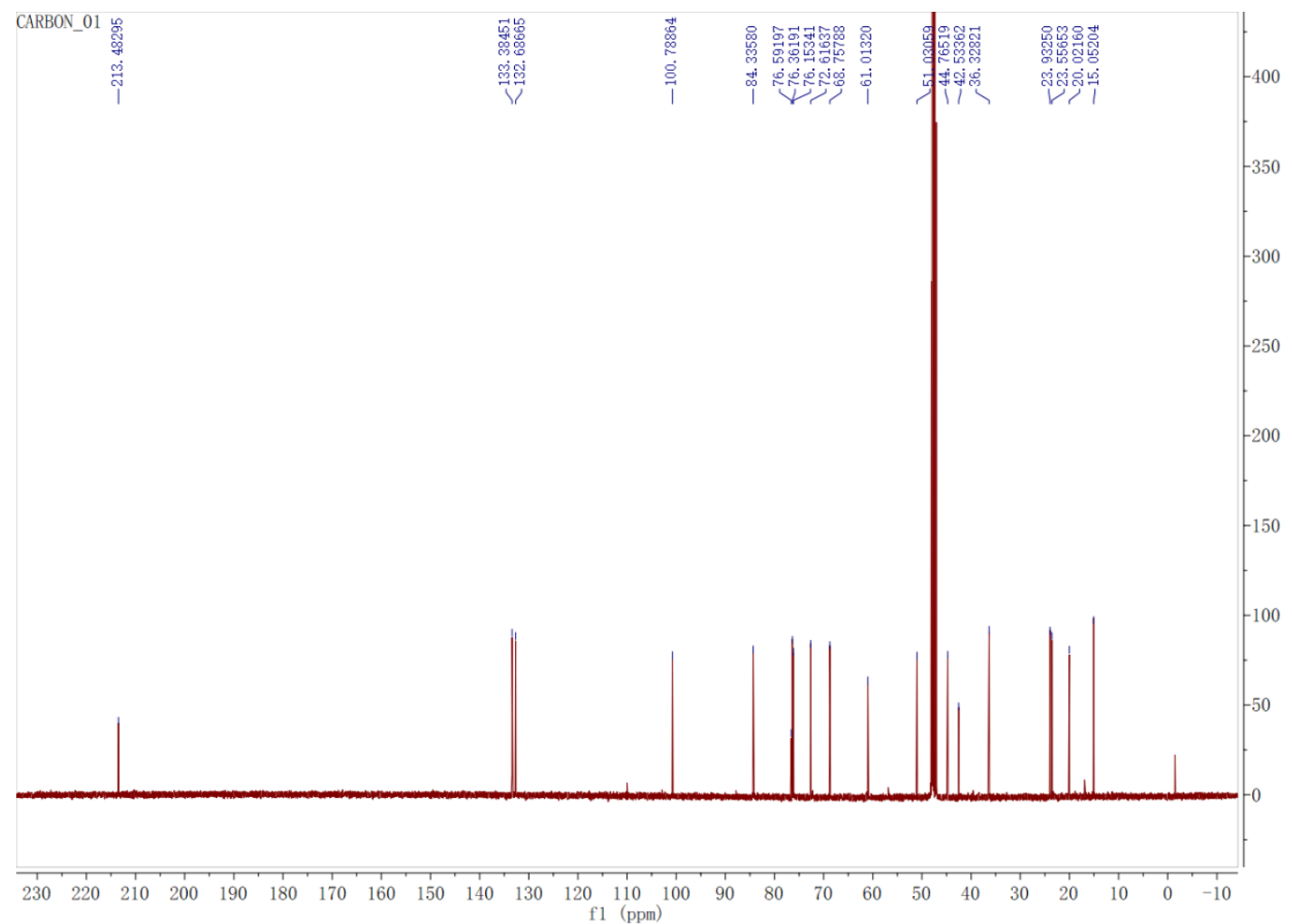

Figure S19. The ${ }^{13} \mathrm{C}$ NMR spectrum of compound 1 in $\mathrm{CDOD}_{3}(500 \mathrm{~Hz})$ 


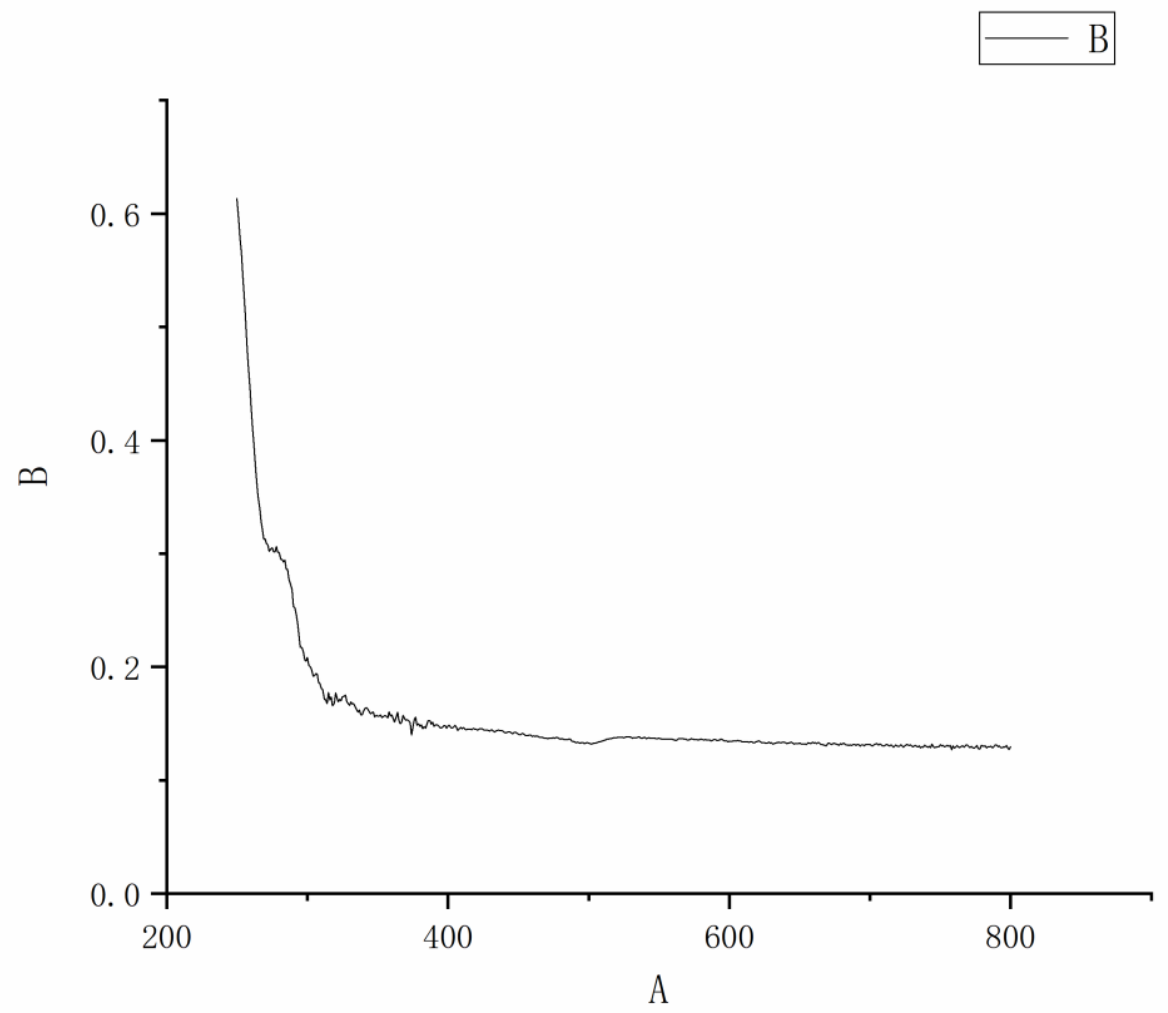

Figure S20. The UV spectra of compound 2.

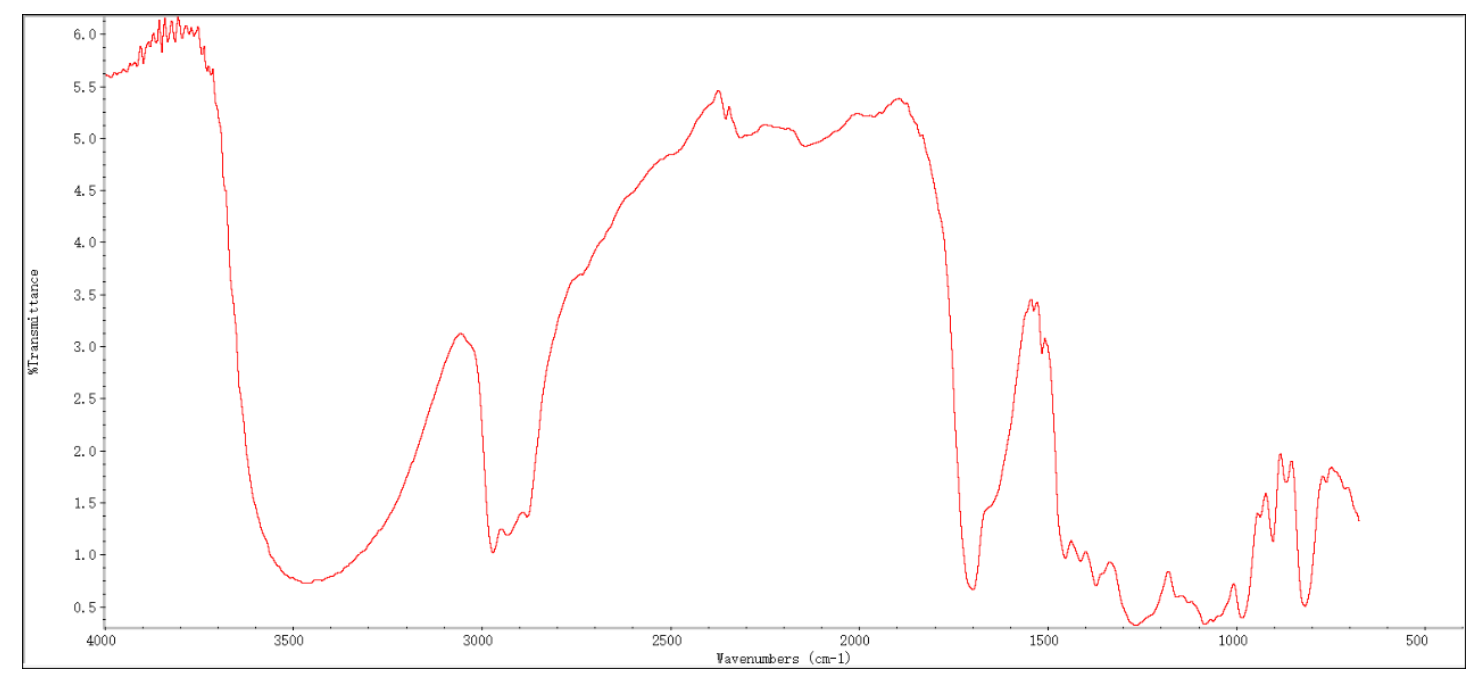

Figure S21. The IR spectra of compound 2 


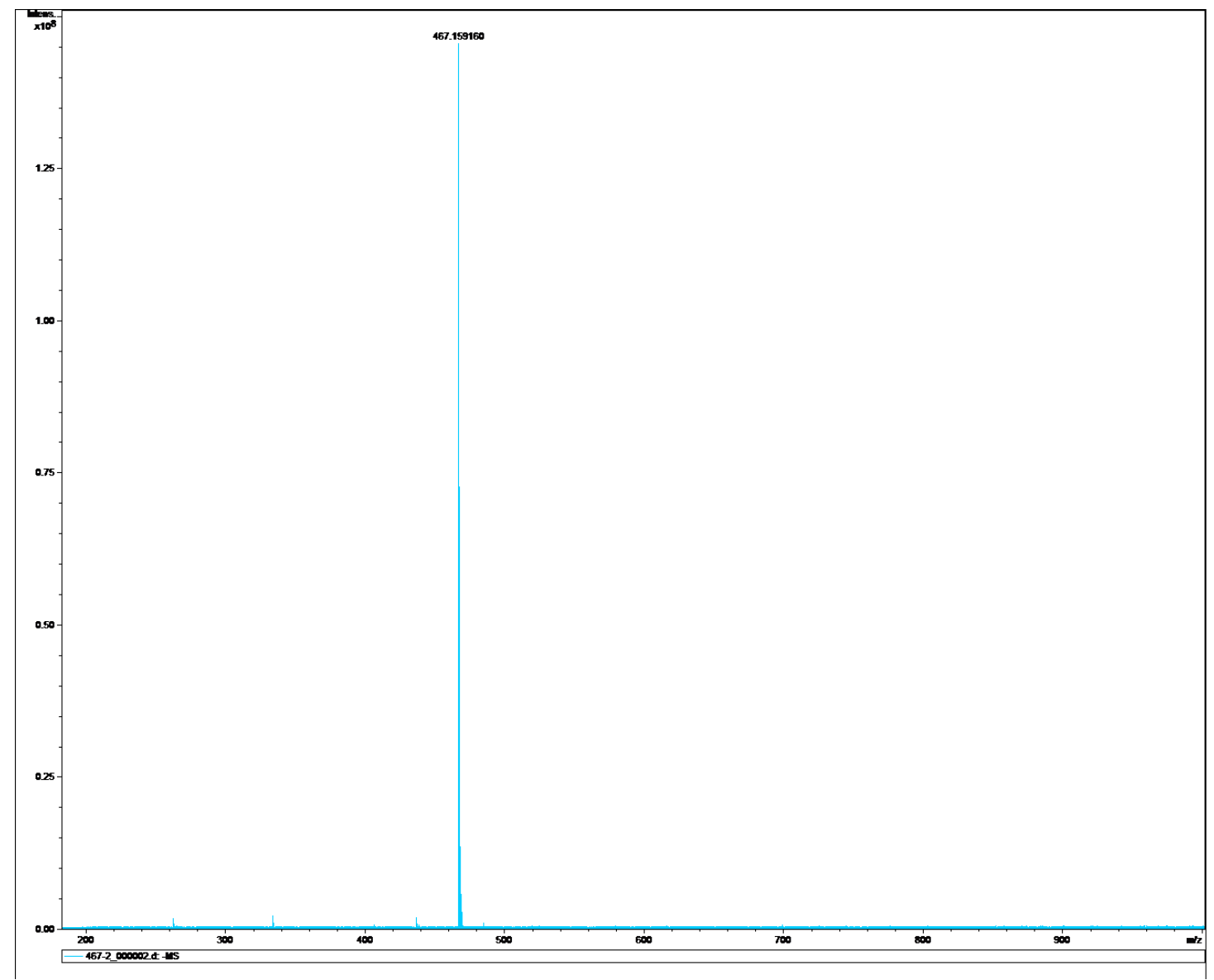

Figure S22. The ESI-TOF-MS spectra of compound 2 


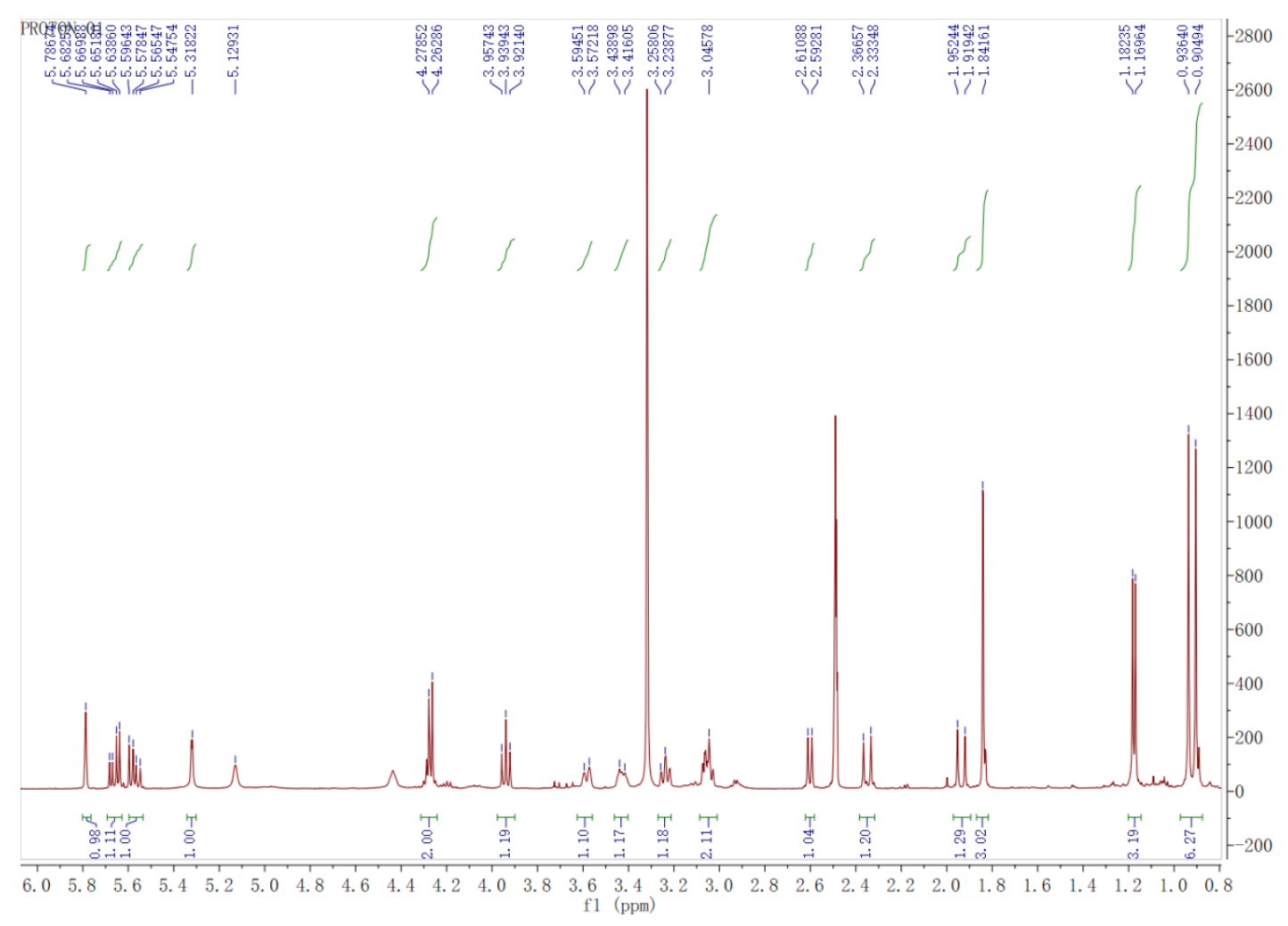

Figure $\mathrm{S} 23$. The ${ }^{1} \mathrm{H}$ NMR spectrum of compound 3 in DMSO-d6 $(500 \mathrm{~Hz})$

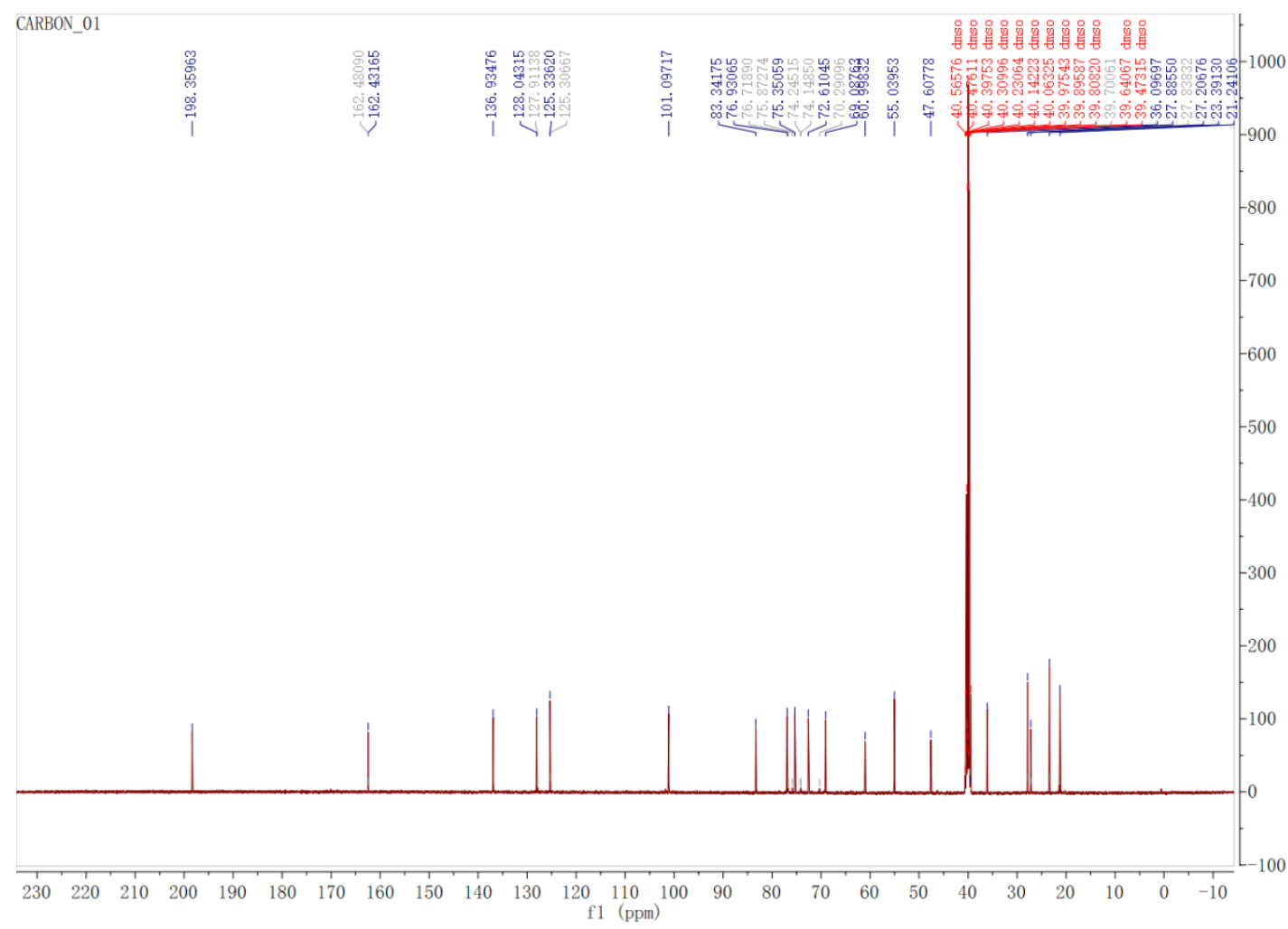

Figure S24. The ${ }^{13} \mathrm{C}$ NMR spectrum of compound 3 in DMSO-d6 $(500 \mathrm{~Hz})$ 


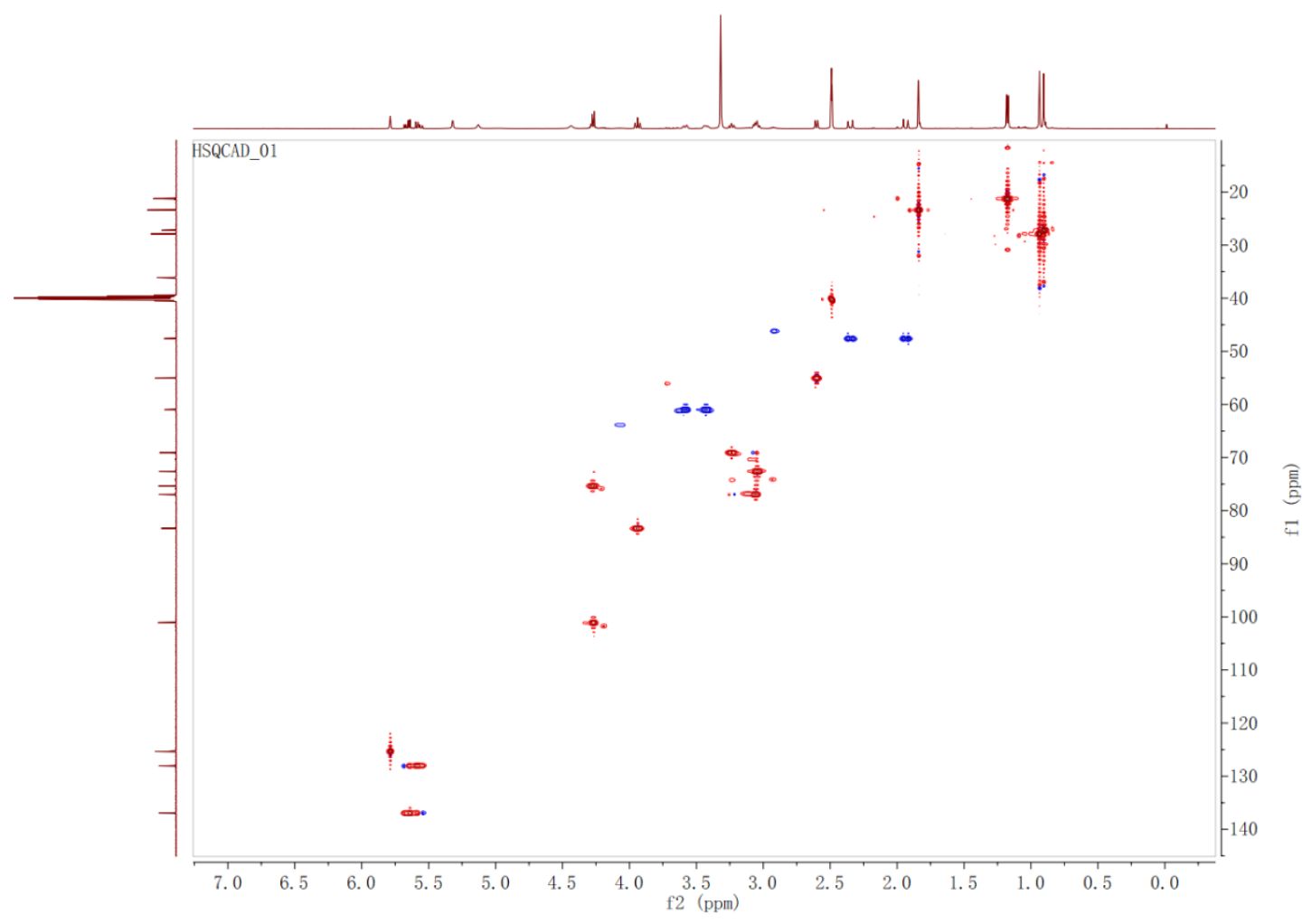

Figure S25. The HSQC spectrum of compound 3 in DMSO-d6 $(500 \mathrm{~Hz})$



Figure S26. The HMBC spectrum of compound 3 in DMSO-d6 $(500 \mathrm{~Hz})$ 


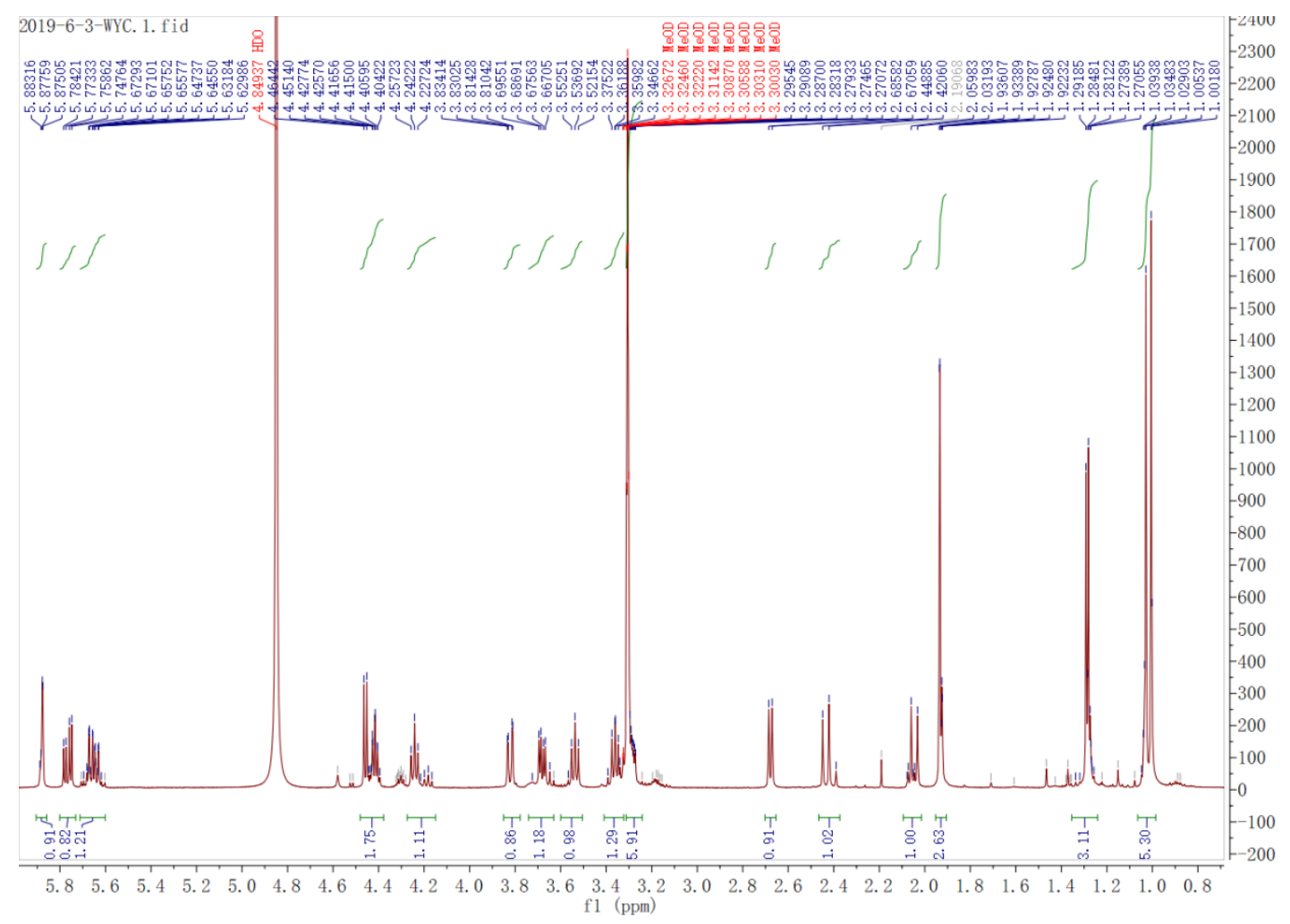

Figure S27. The 1H NMR spectrum of compound 3 in CDOD3 $(500 \mathrm{~Hz})$

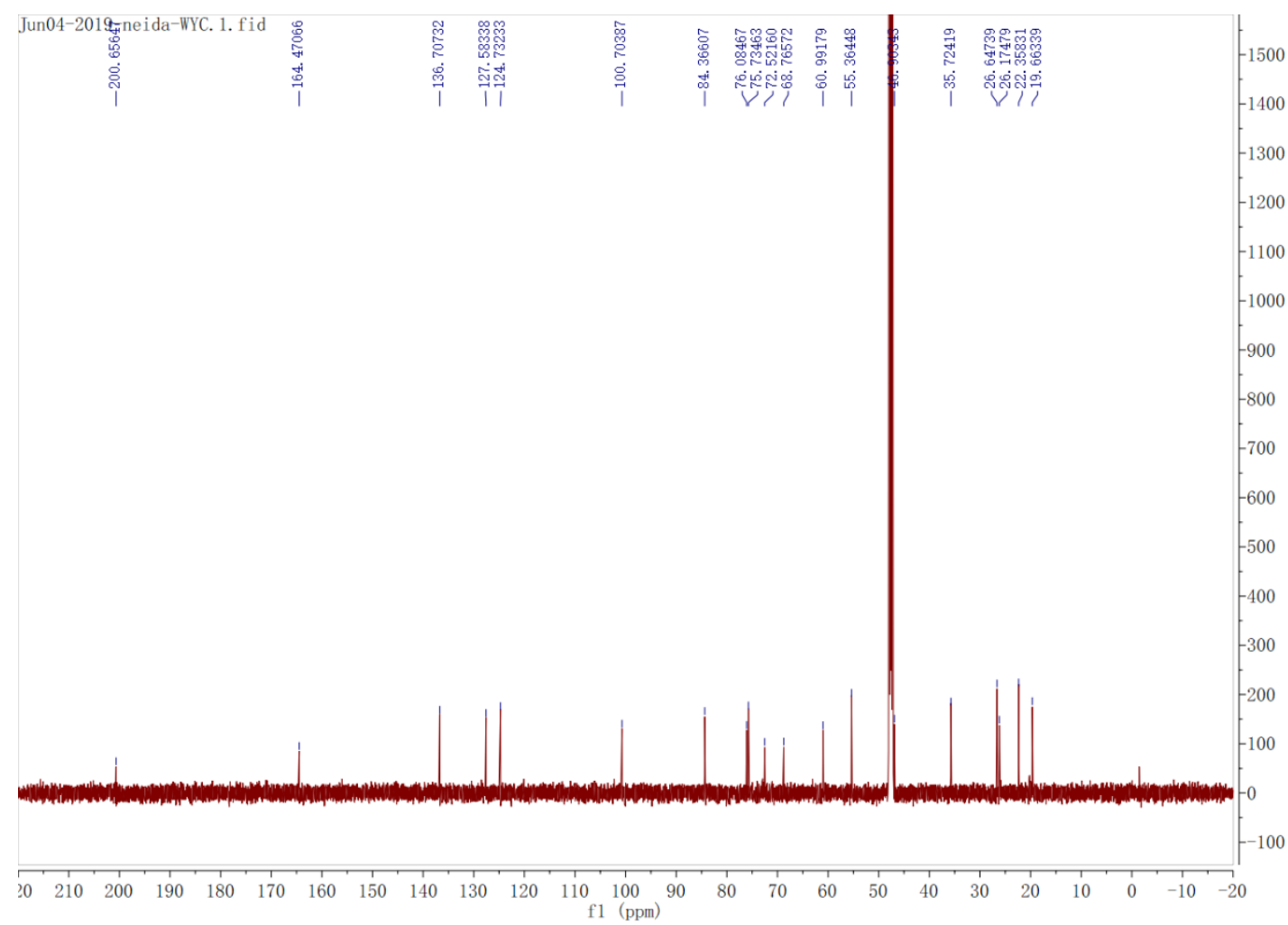

Figure S28. The 13C NMR spectrum of compound 3 in CDOD3 $(500 \mathrm{~Hz})$ 




Figure S29. The UV spectra of compound 3

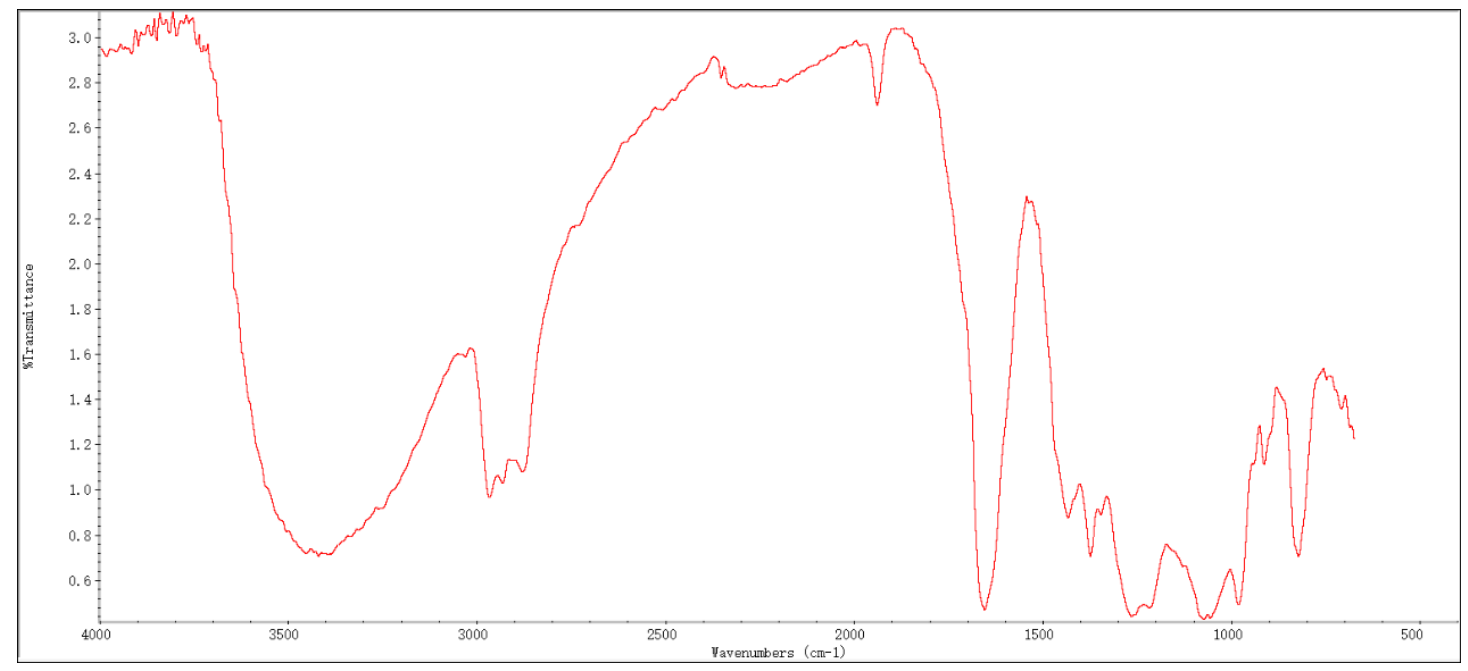

Figure S30. The IR spectra of compound 3 


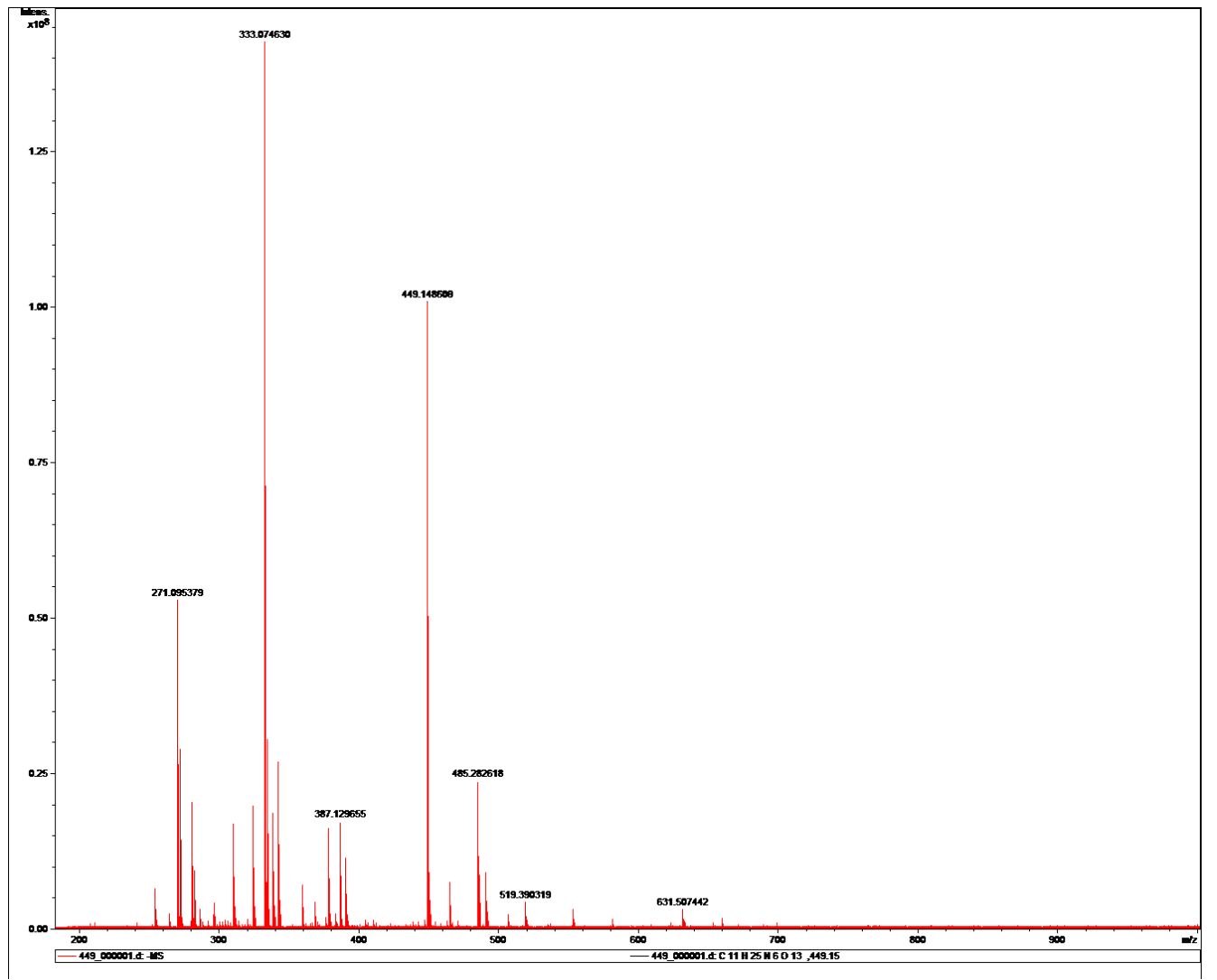

Figure S31. The ESI-TOF-MS spectra of compound 3

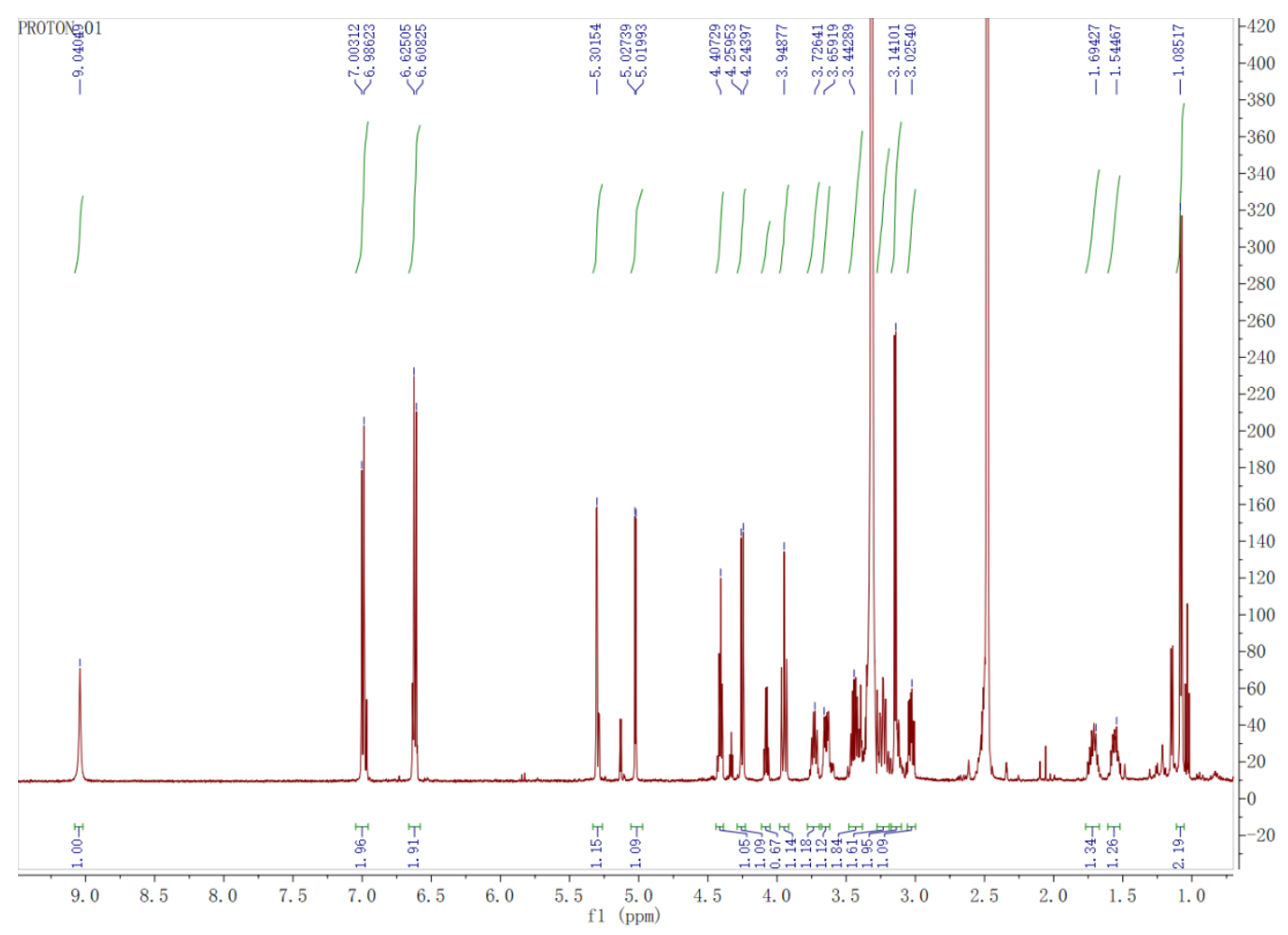

Figure S32. The ${ }^{1} \mathrm{H}$ NMR spectrum of compound 4 in DMSO-d6 $(500 \mathrm{~Hz})$ 




Figure S33. The ${ }^{13} \mathrm{C}$ NMR spectrum of compound 4 in DMSO- $d 6(500 \mathrm{~Hz})$

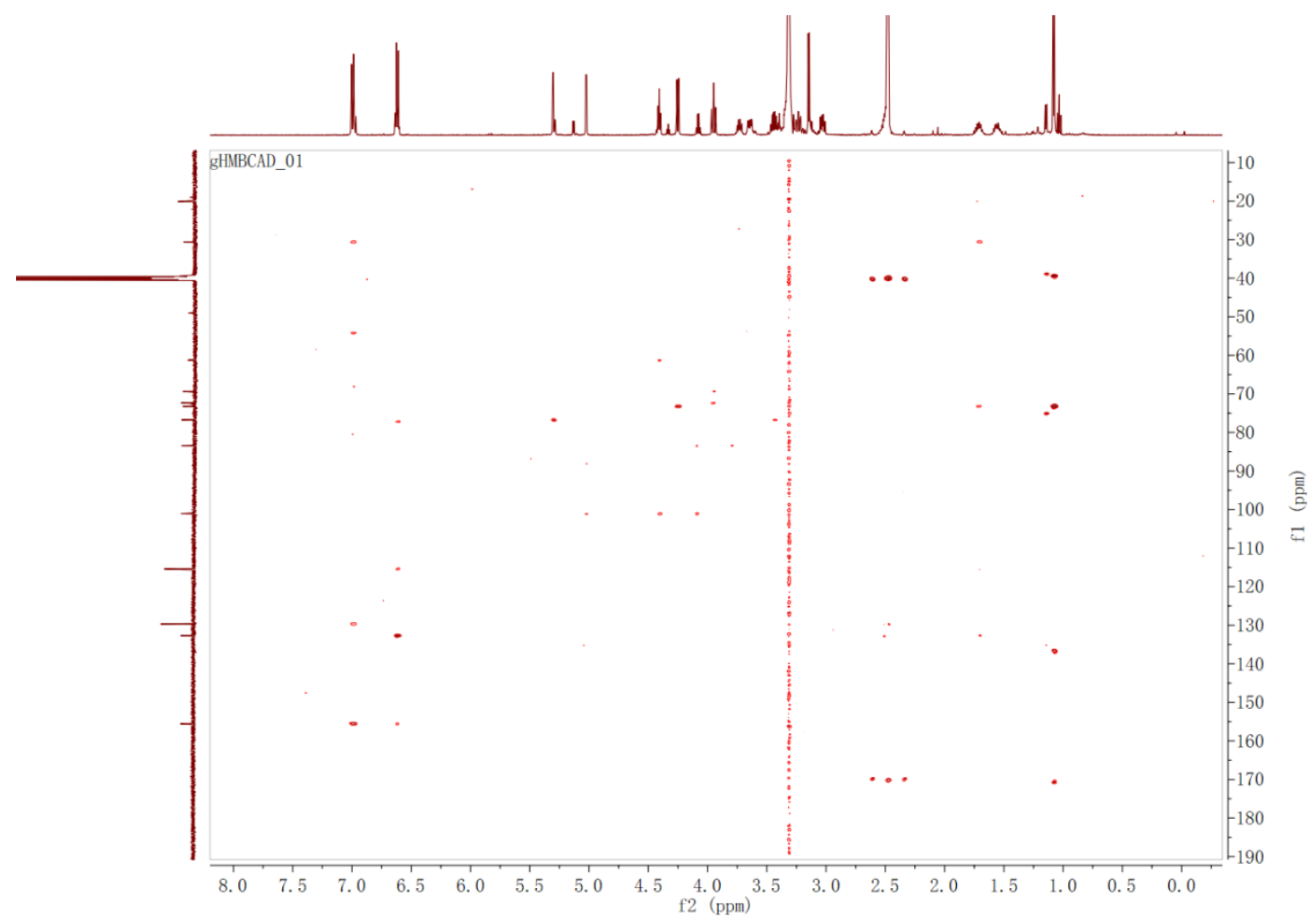

Figure S34. The HMBC spectrum of compound 4 in DMSO-d6 $(500 \mathrm{~Hz})$ 


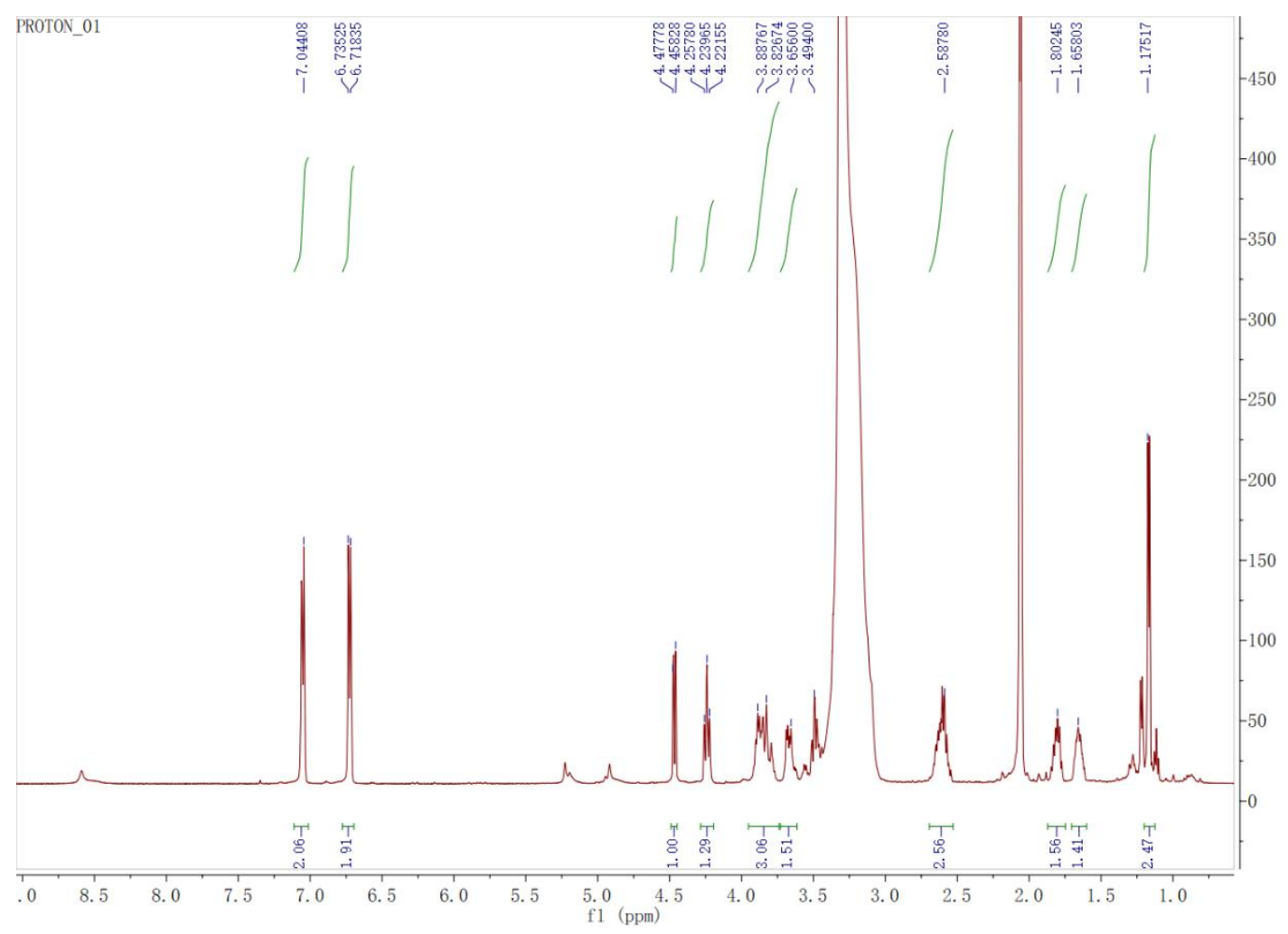

Figure S35. The ${ }^{1} \mathrm{H}$ NMR spectrum of compound 4 in Acetone-d6 $(500 \mathrm{~Hz})$

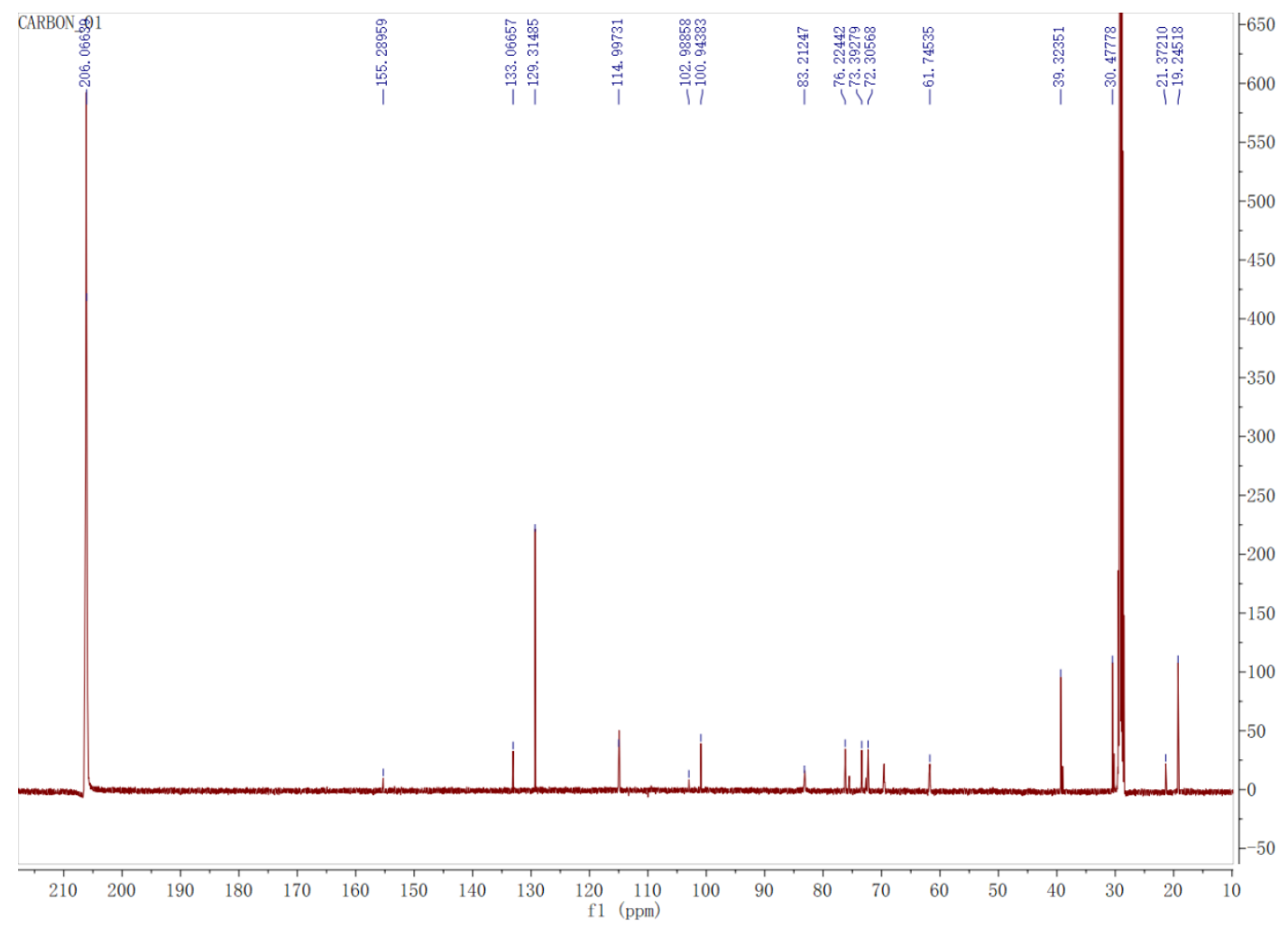

Figure S36. The ${ }^{13} \mathrm{C}$ NMR spectrum of compound 4 in Acetone- $d 6(500 \mathrm{~Hz})$ 




Figure S37. The HSQC spectrum of compound 4 in Acetone-d6 $(500 \mathrm{~Hz})$

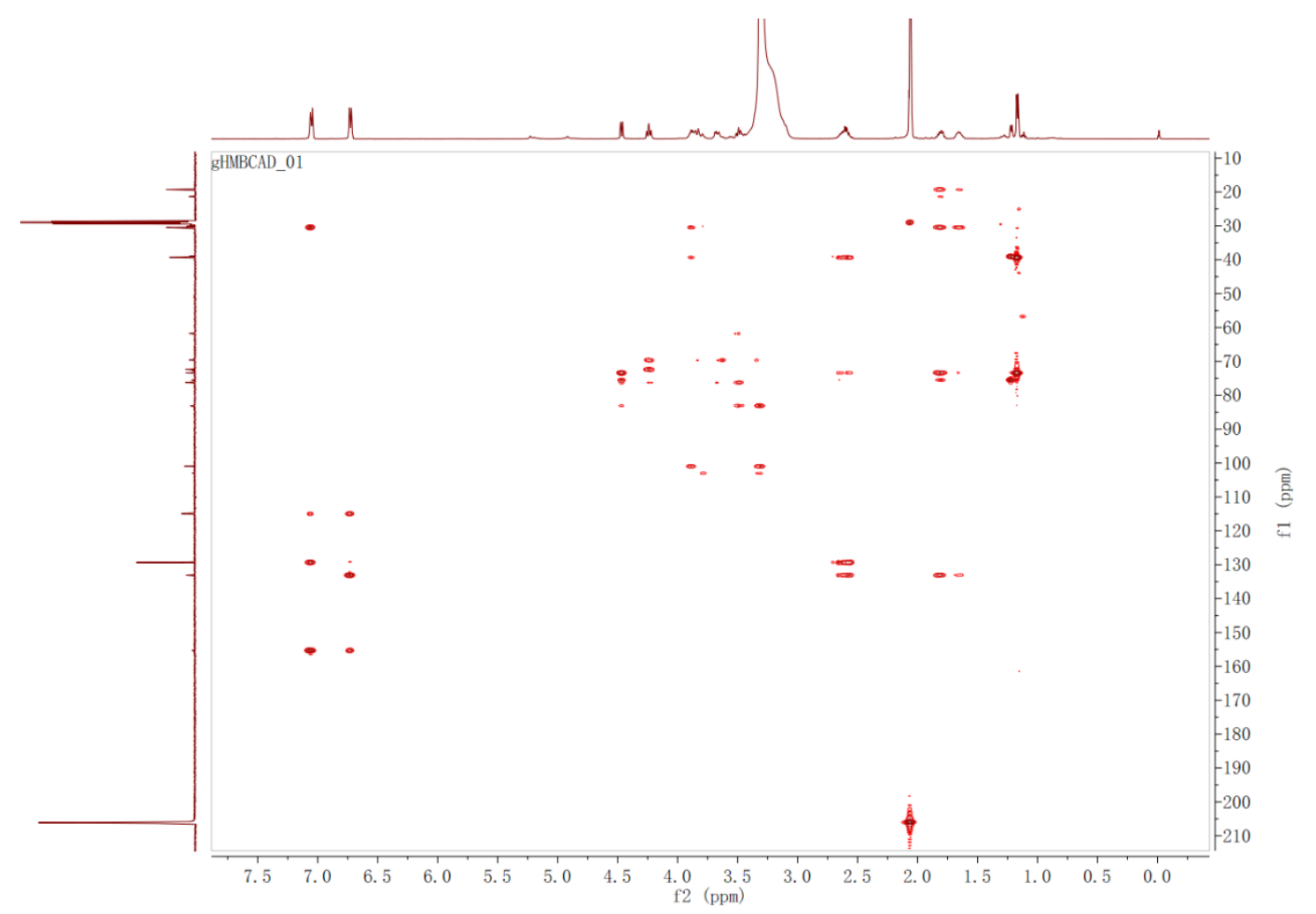

Figure S38. The HMBC spectrum of compound 4 in Acetone-d6 $(500 \mathrm{~Hz})$ 


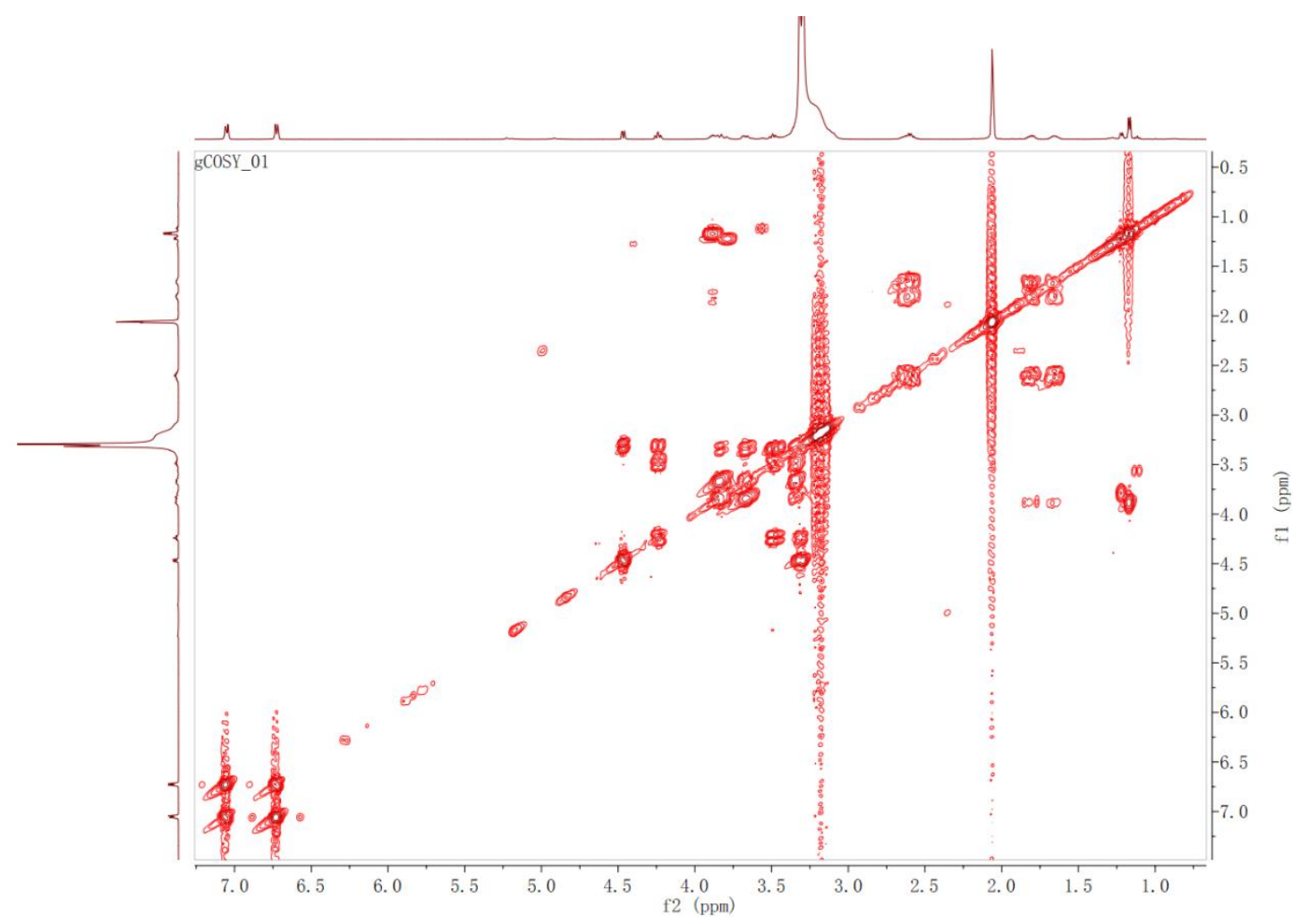

Figure S39. The ${ }^{1} \mathrm{H}-{ }^{1} \mathrm{H}$ COSY spectrum of compound 4 in Acetone- $d 6(500 \mathrm{~Hz})$

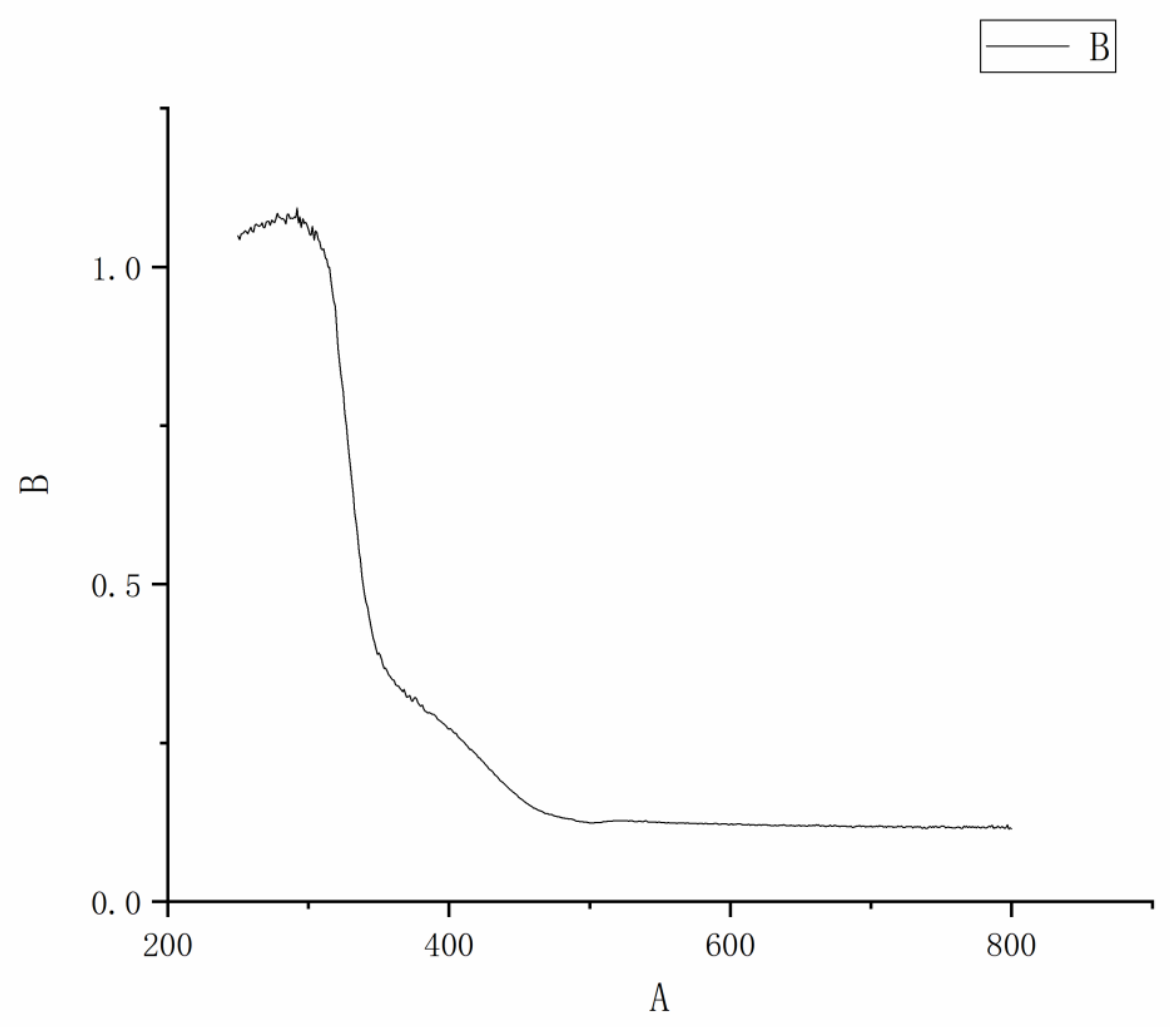


The UV spectra of compound 4

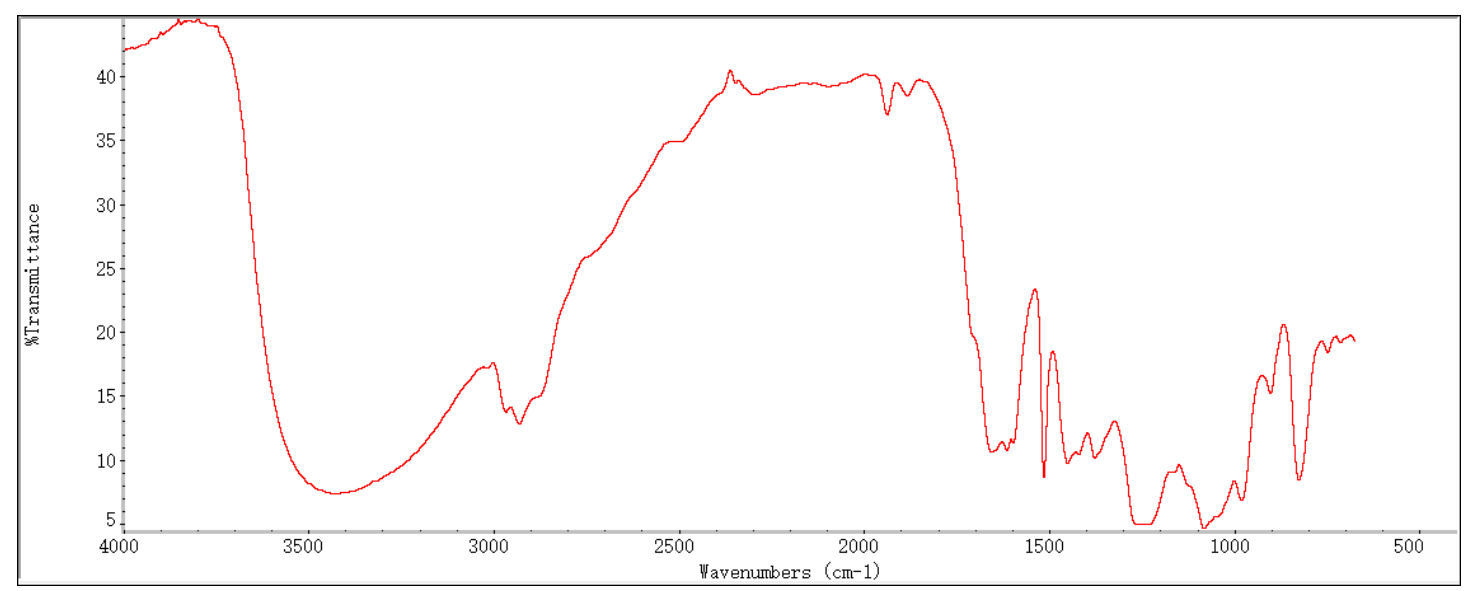

Figure S40. The IR spectra of compound 4

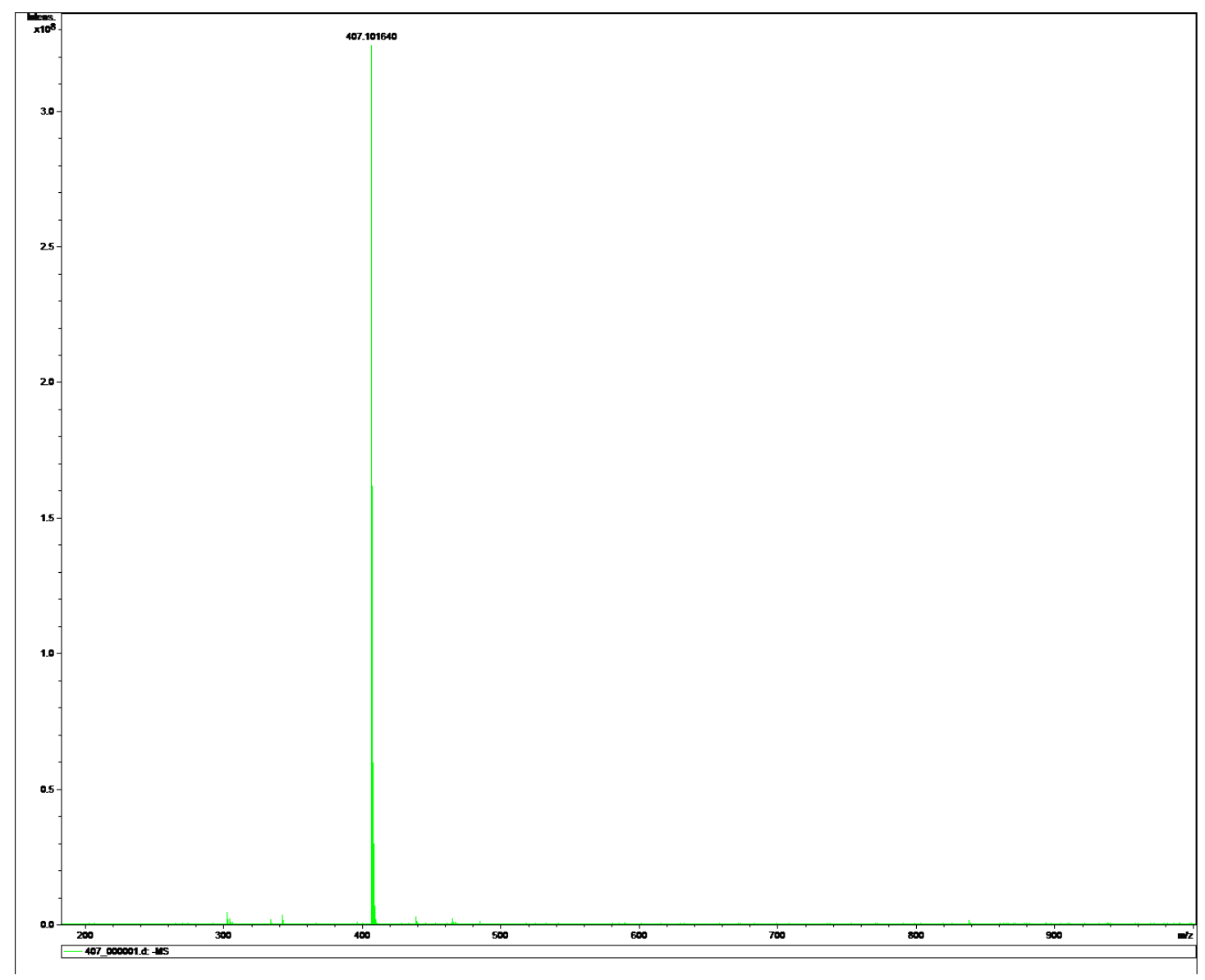

Figure S41. The ESI-TOF-MS spectra of compound 4 


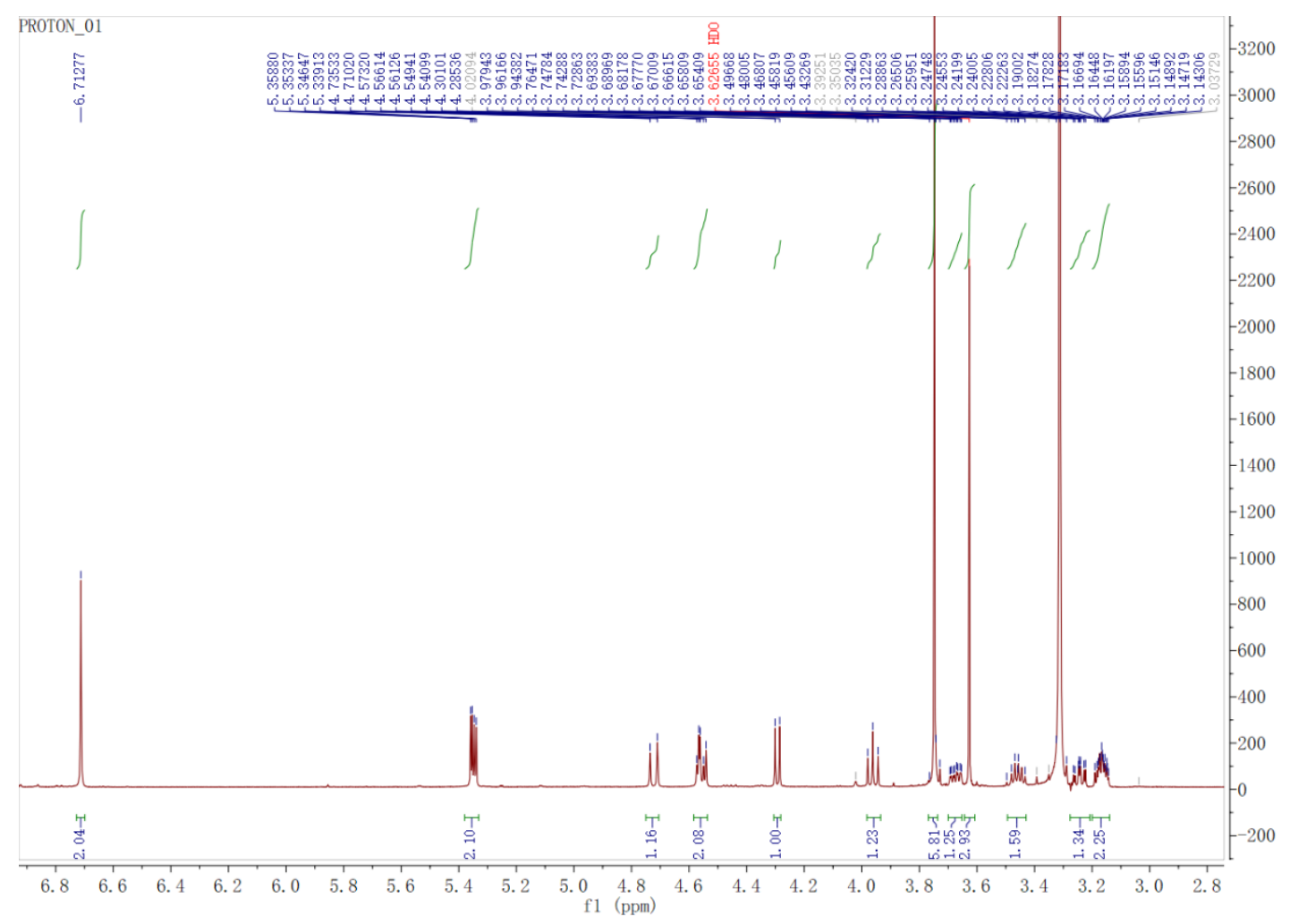

Figure S42. The ${ }^{1} \mathrm{H}$ NMR spectrum of compound 5 in DMSO-d6 $(500 \mathrm{~Hz})$

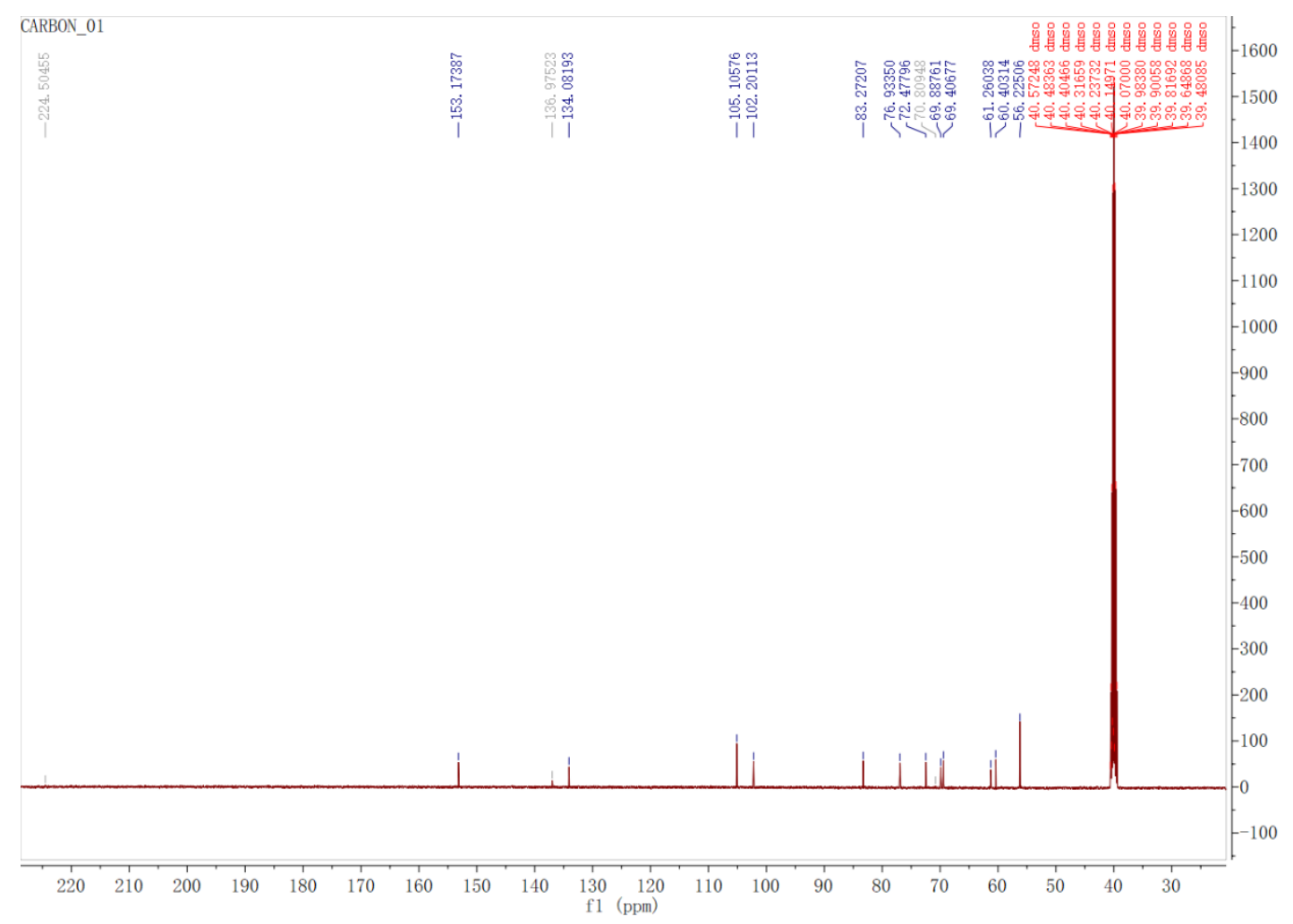

Figure S43. The ${ }^{13} \mathrm{C}$ NMR spectrum of compound 5 in DMSO-d6 $(500 \mathrm{~Hz})$ 


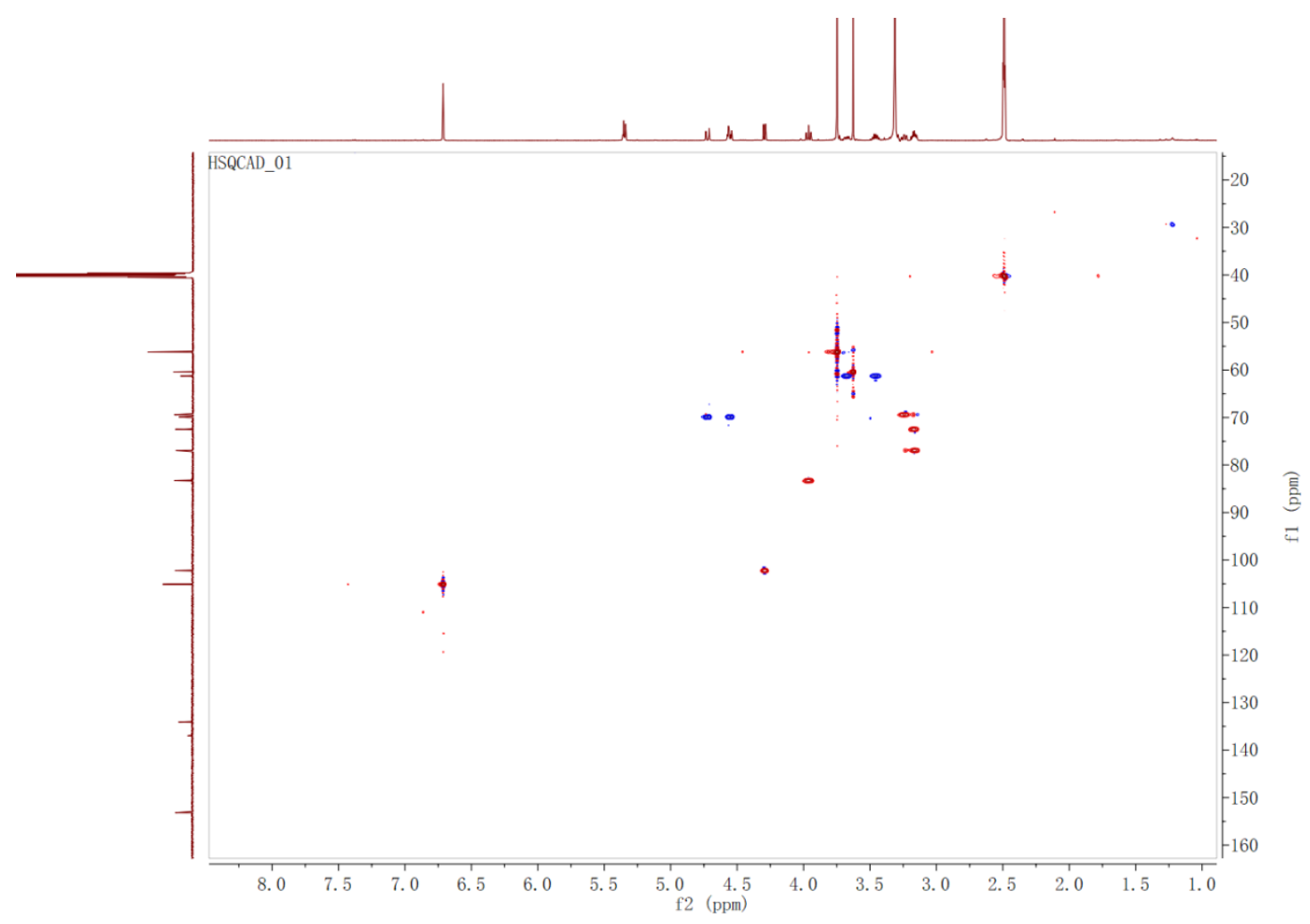

Figure S44. The HSQC spectrum of compound 5 in DMSO-d6 $(500 \mathrm{~Hz})$

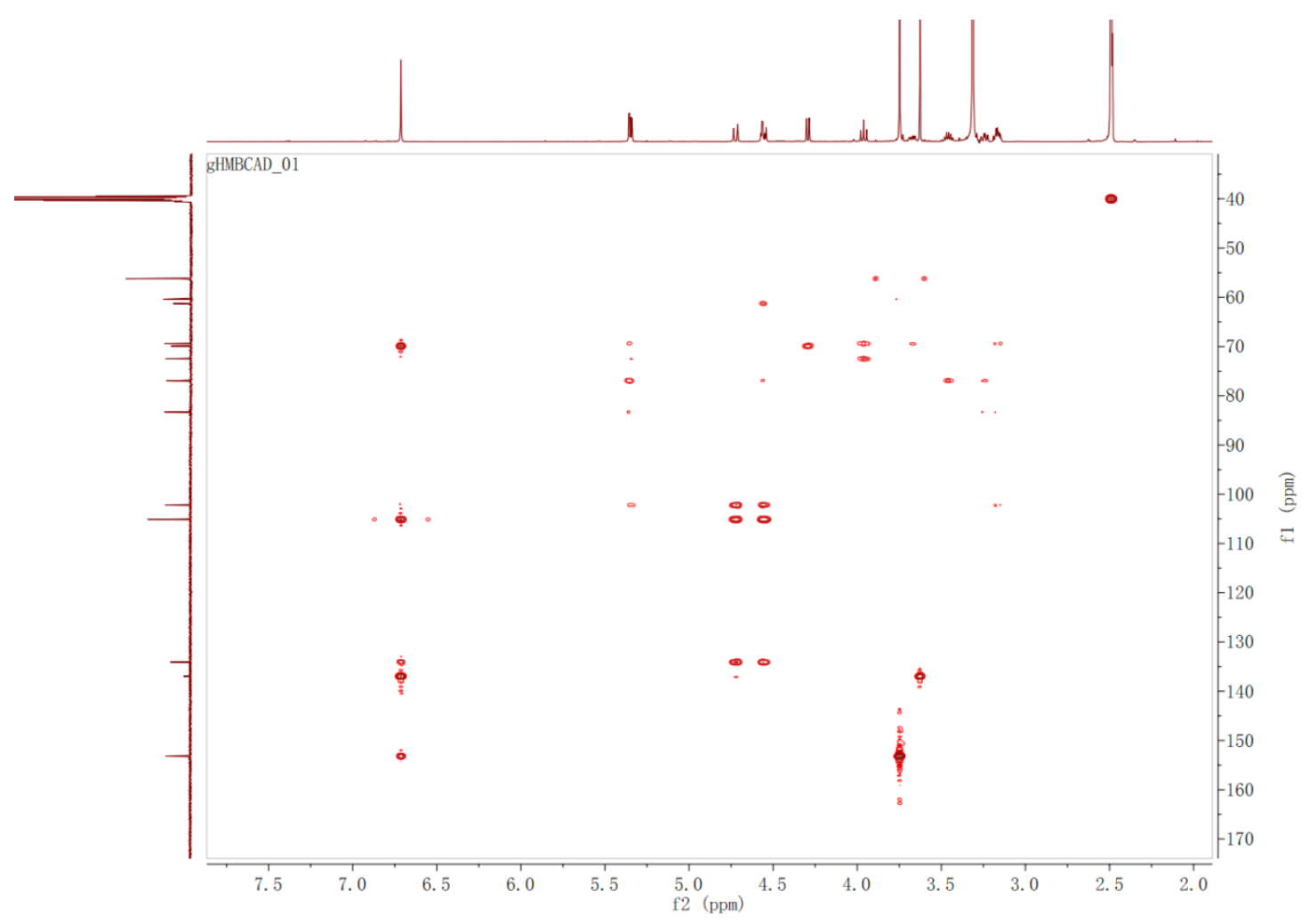

Figure S45. The HMBC spectrum of compound 5 in DMSO-d6 $(500 \mathrm{~Hz})$ 


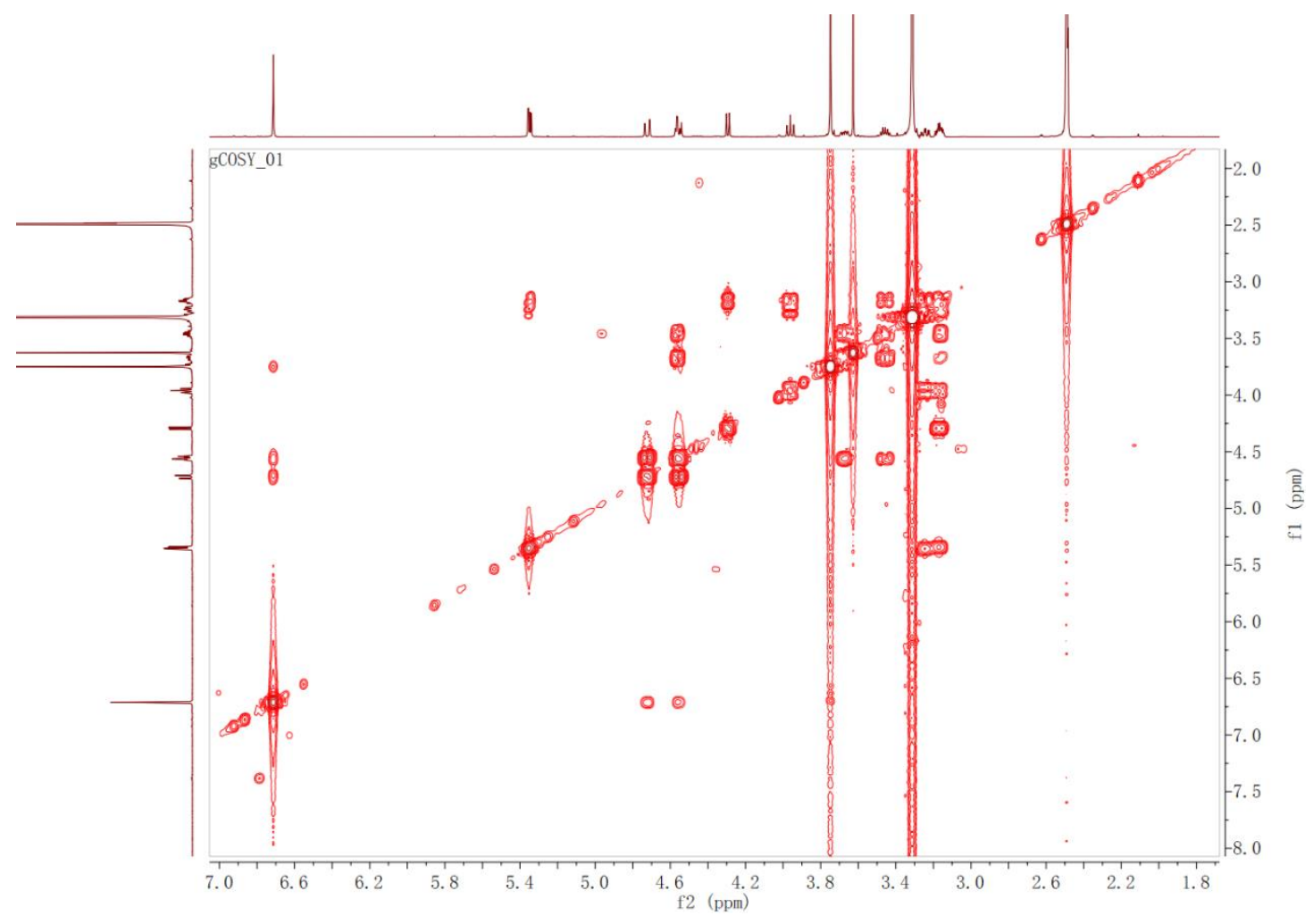

Figure S46. The ${ }^{1} \mathrm{H}-{ }^{1} \mathrm{H}$ COSY spectrum of compound 5 in DMSO- $d 6(500 \mathrm{~Hz})$

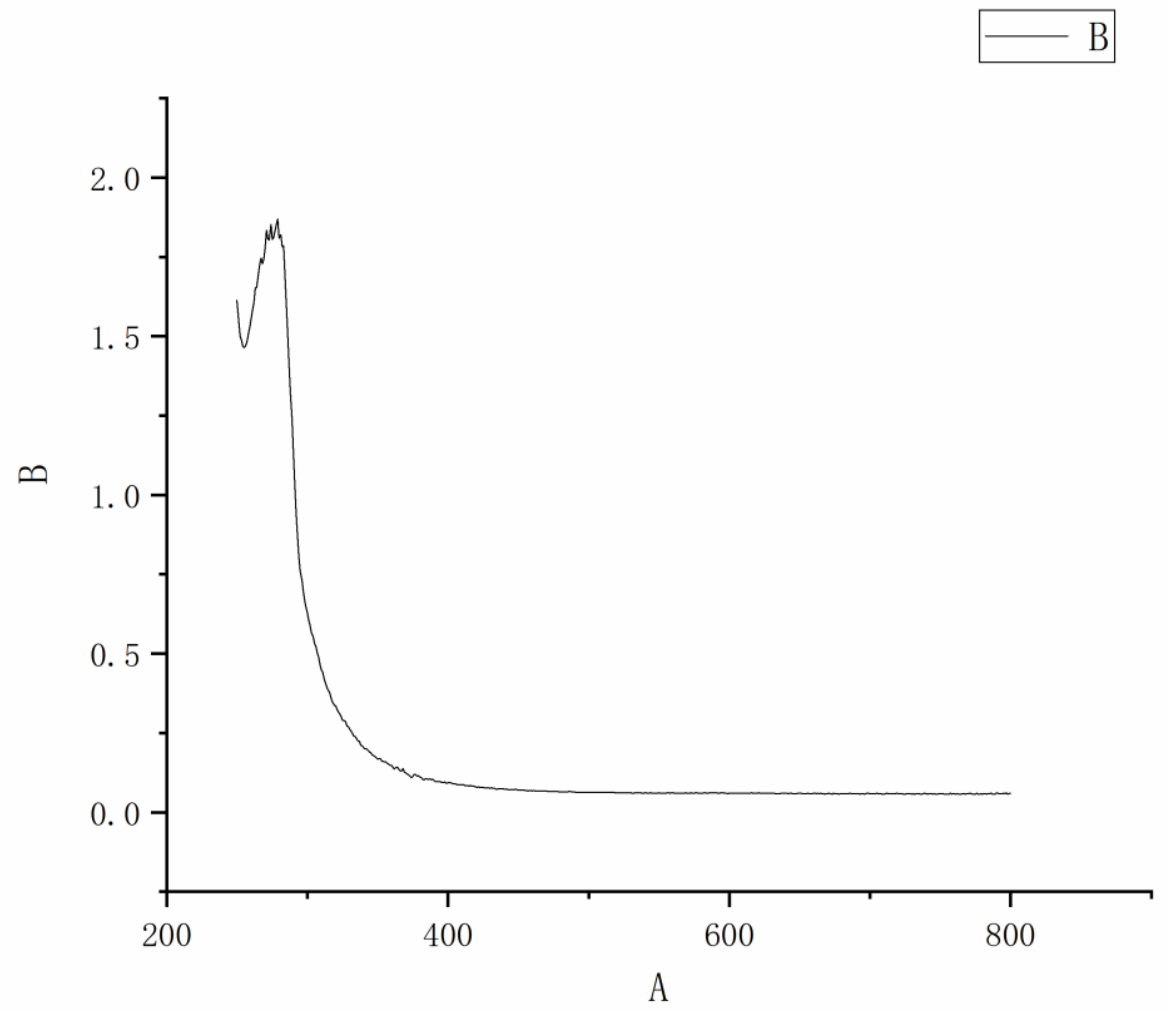

Figure S47. The UV spectra of compound 5 


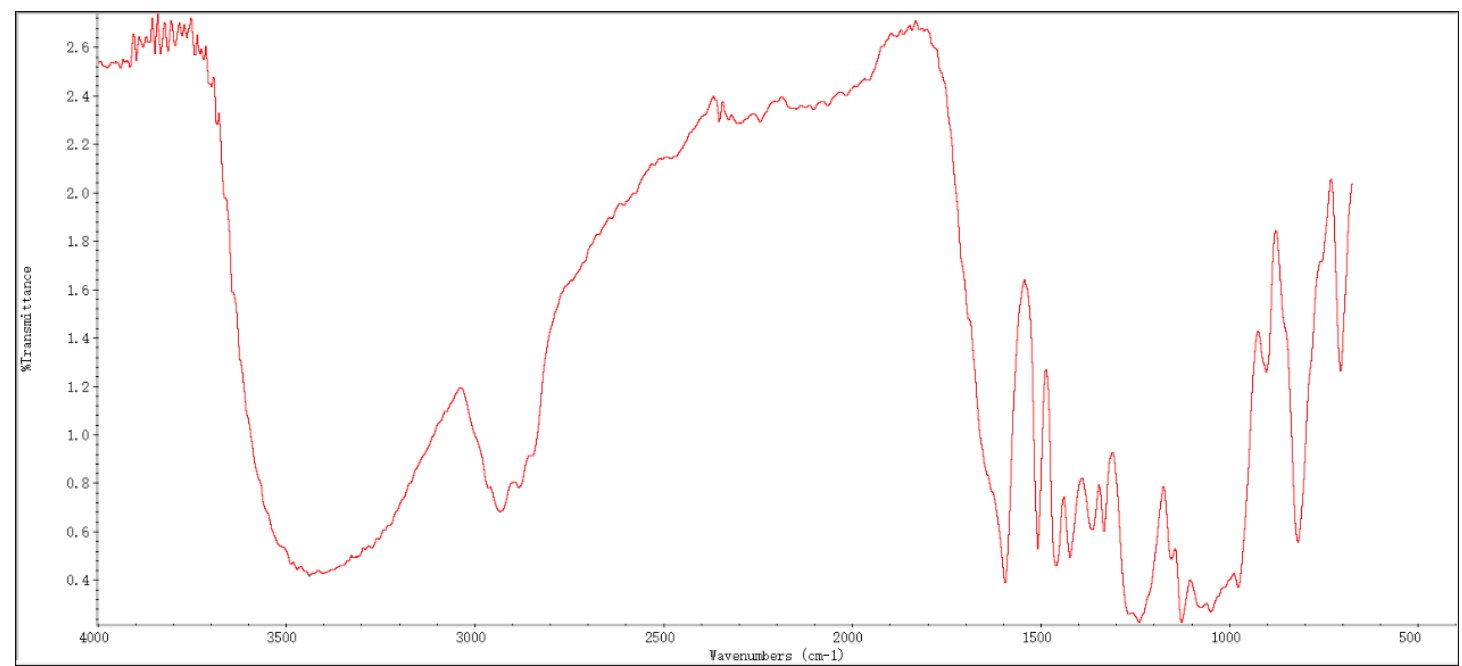

Figure S48. The IR spectra of compound 5

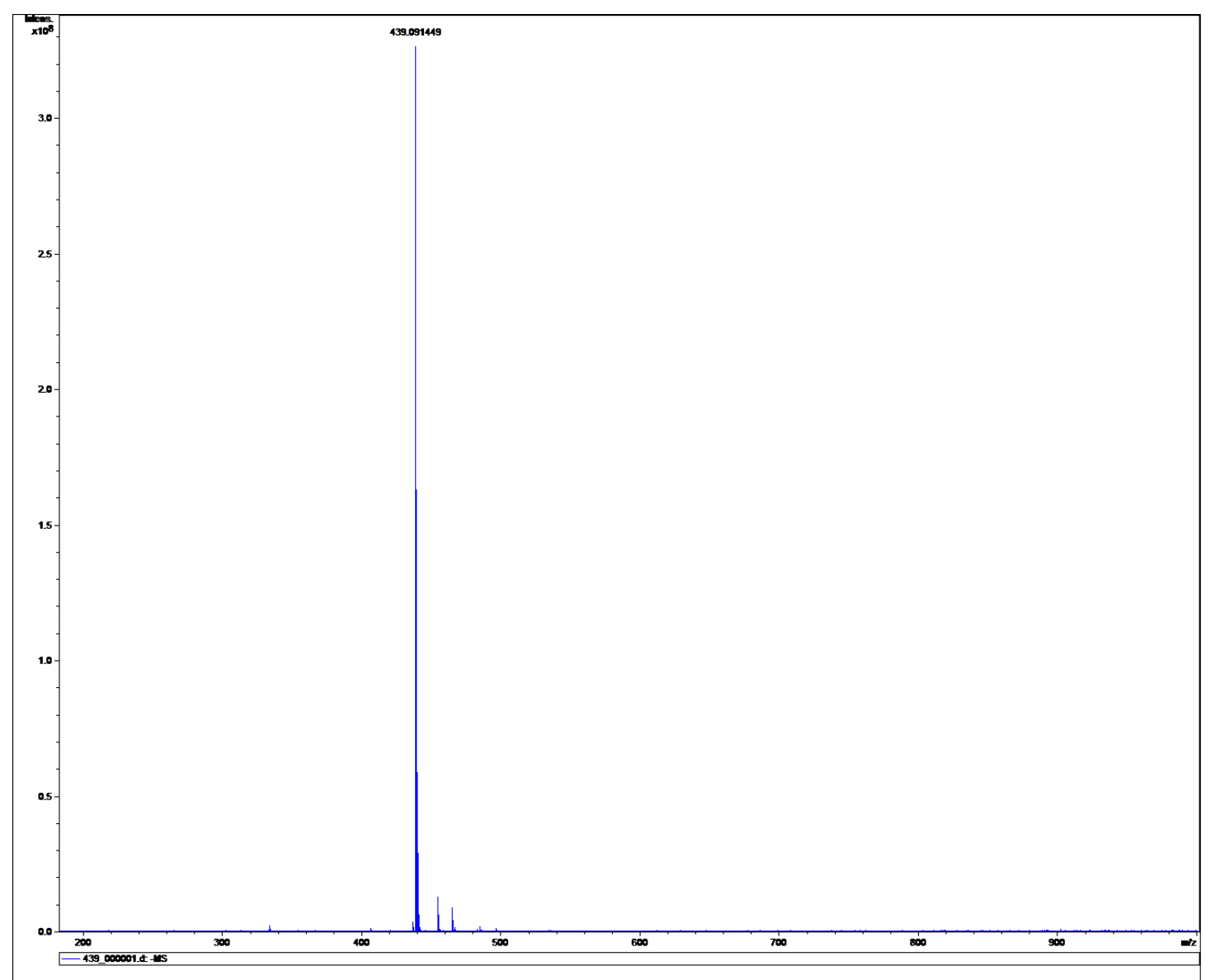

Figure S49. The ESI-TOF-MS spectra of compound 5 


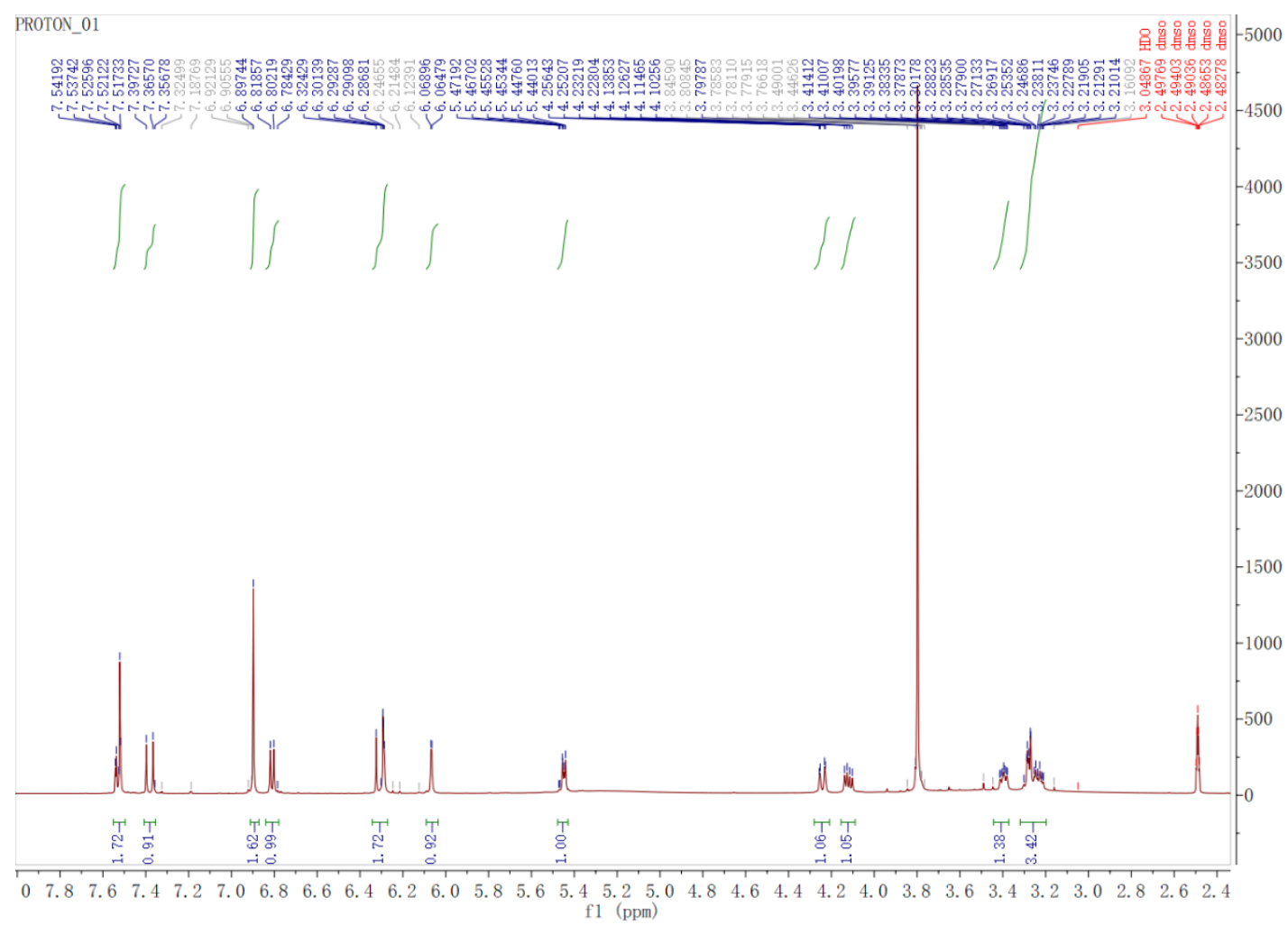

Figure S50. The ${ }^{13} \mathrm{C}$ NMR spectrum of compound 15 in DMSO-d6 $(500 \mathrm{~Hz})$ 


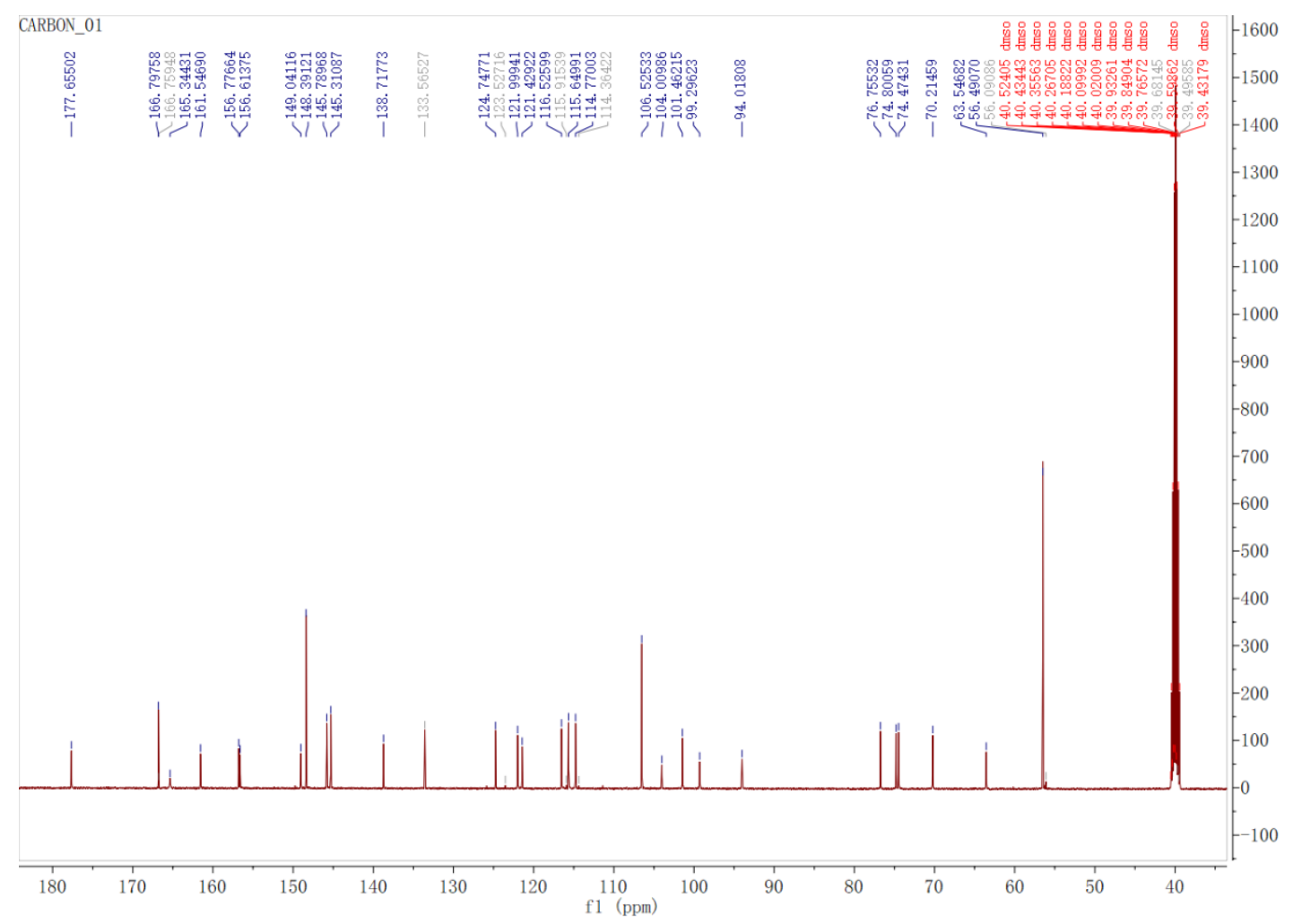

Figure S51. The ${ }^{13} \mathrm{C}$ NMR spectrum of compound 15 in DMSO-d6 $(500 \mathrm{~Hz})$

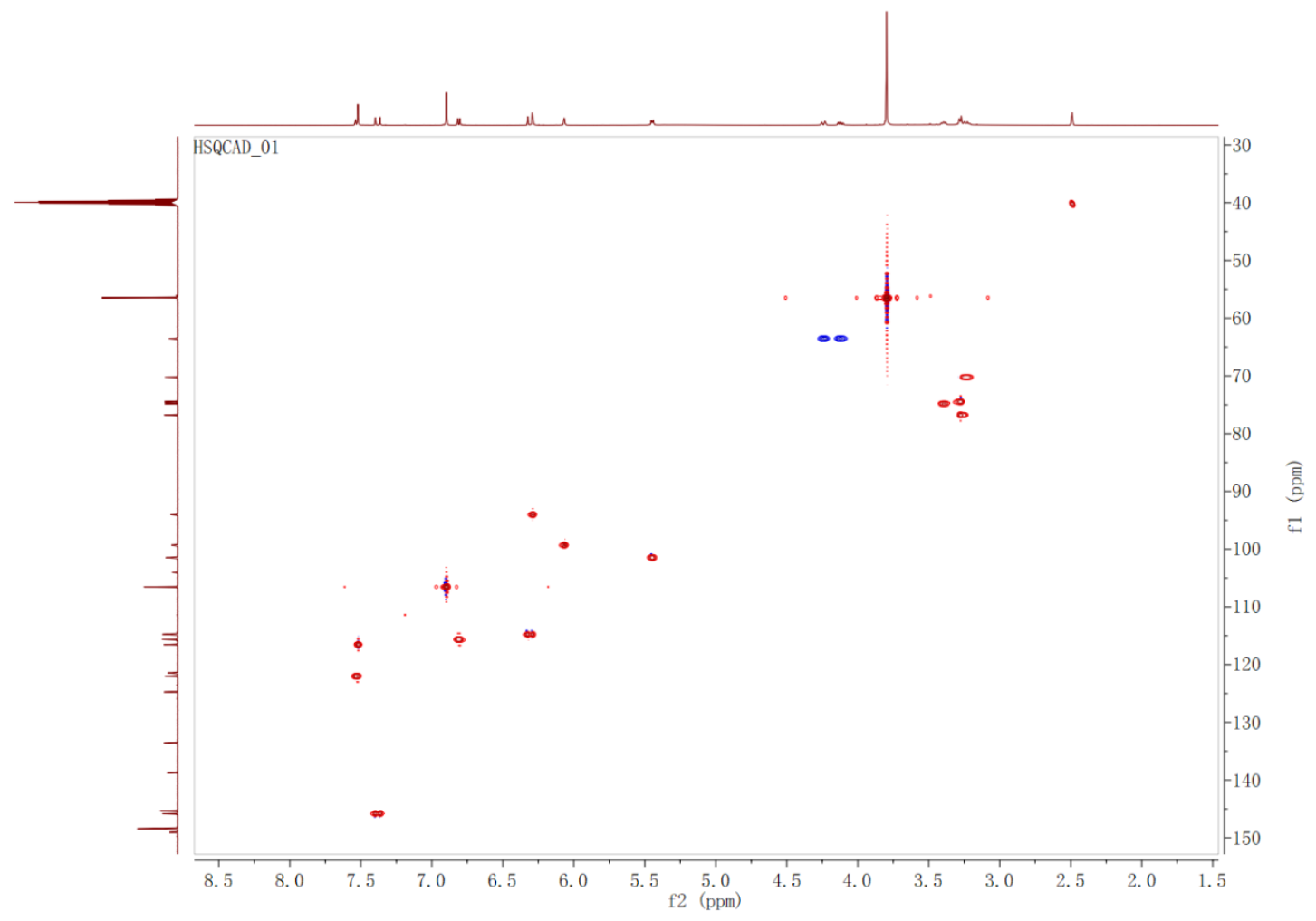

Figure S52. The HSQC spectrum of compound 15 in DMSO-d6 $(500 \mathrm{~Hz})$ 




Figure S53. The HMBC spectrum of compound 15 in DMSO-d6 $(500 \mathrm{~Hz})$

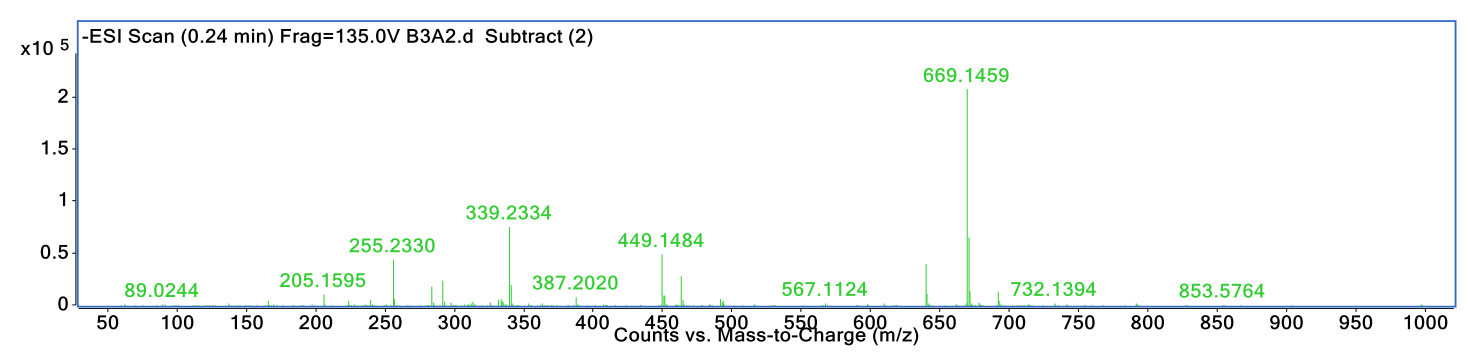

Figure S54. The ESI-TOF-MS spectra of compound 15

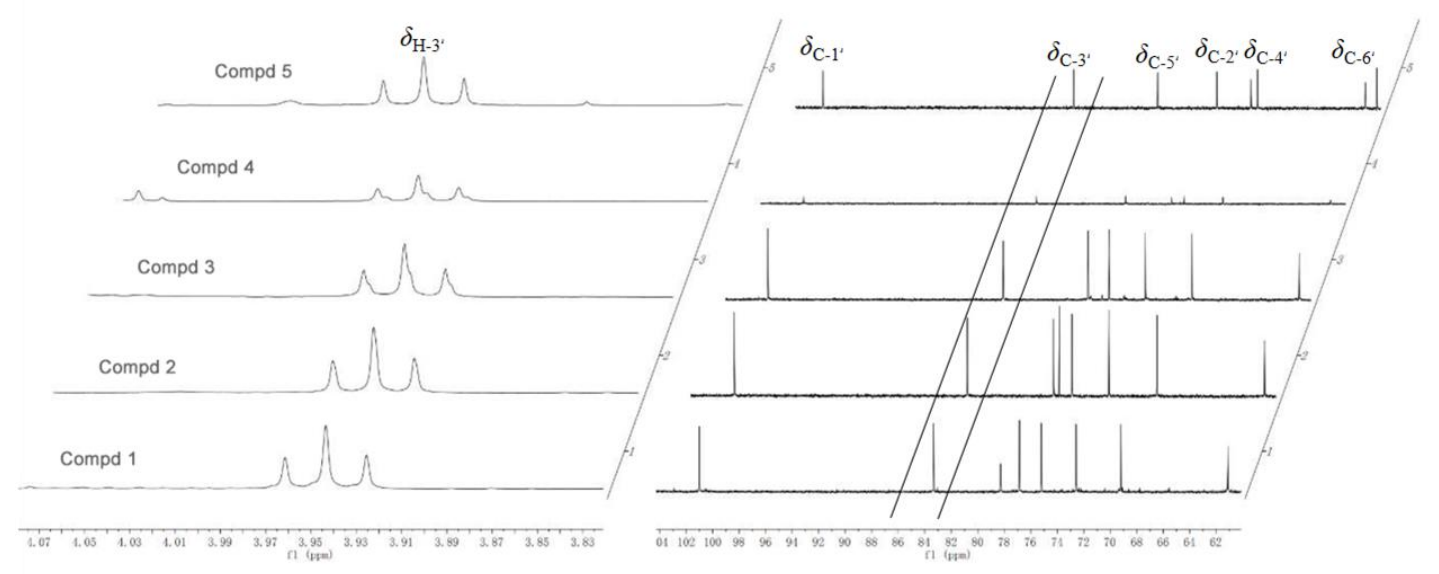

Figure S55. The chemical shift $\left(\delta_{H-3^{\prime}}, \delta_{C-l^{\prime}}-\delta_{C-6^{\prime}}\right)$ of the glucose moiety in compounds 1-5. 


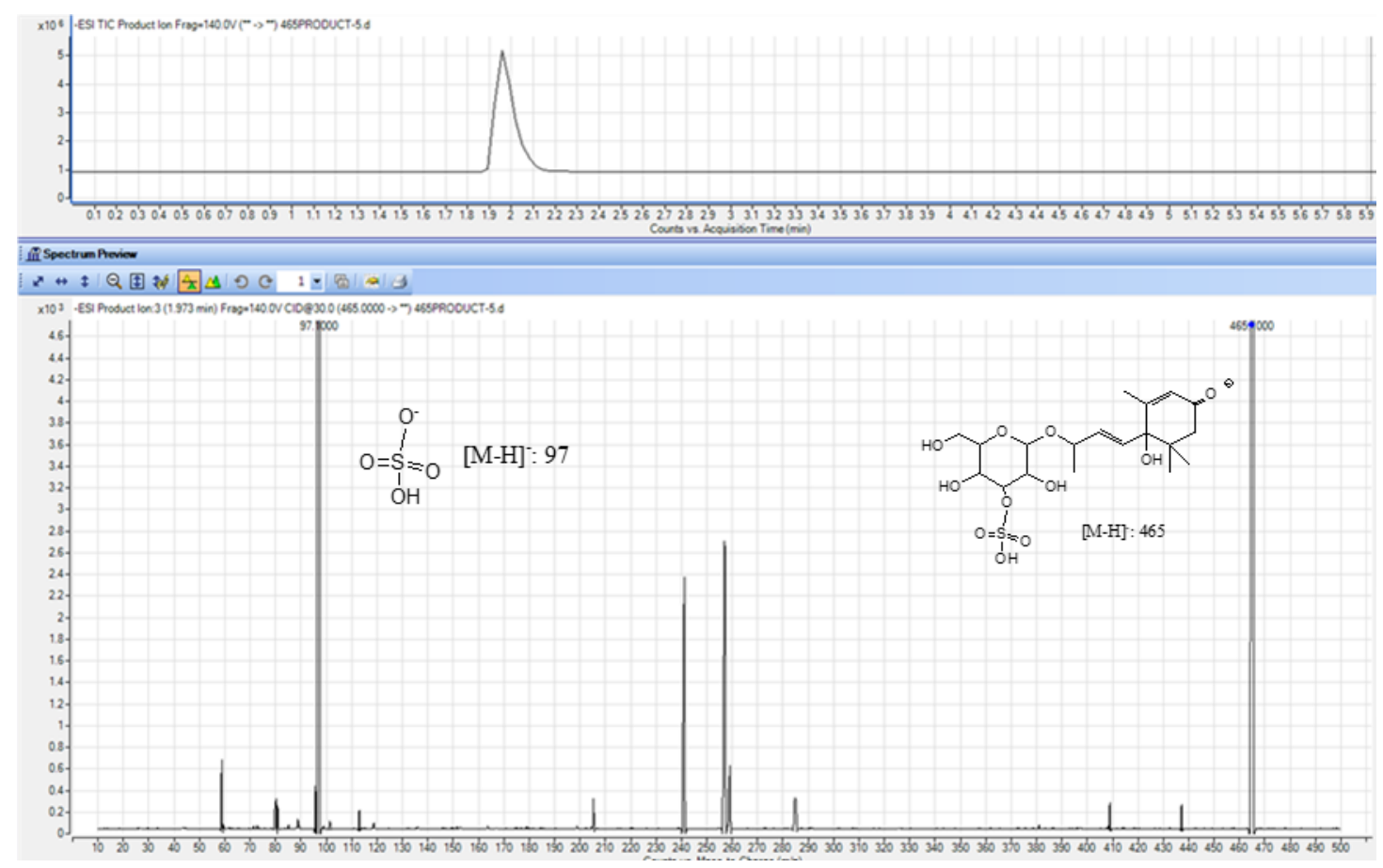

Figure S56. MS/MS spectra of compound 1

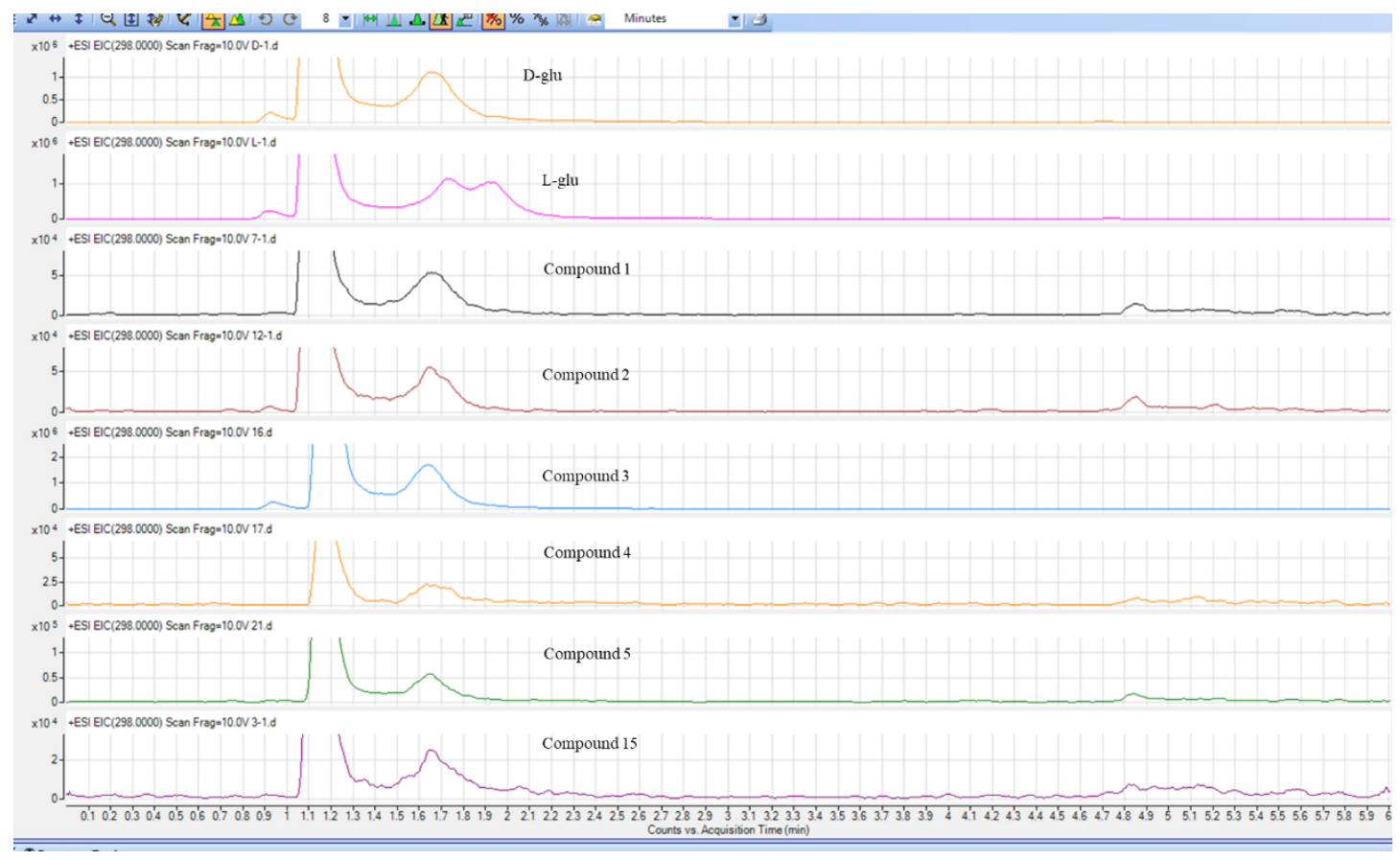

Figure S57. LC-MS data for the determination of sugar moiety of compounds 1-5 and 15. 


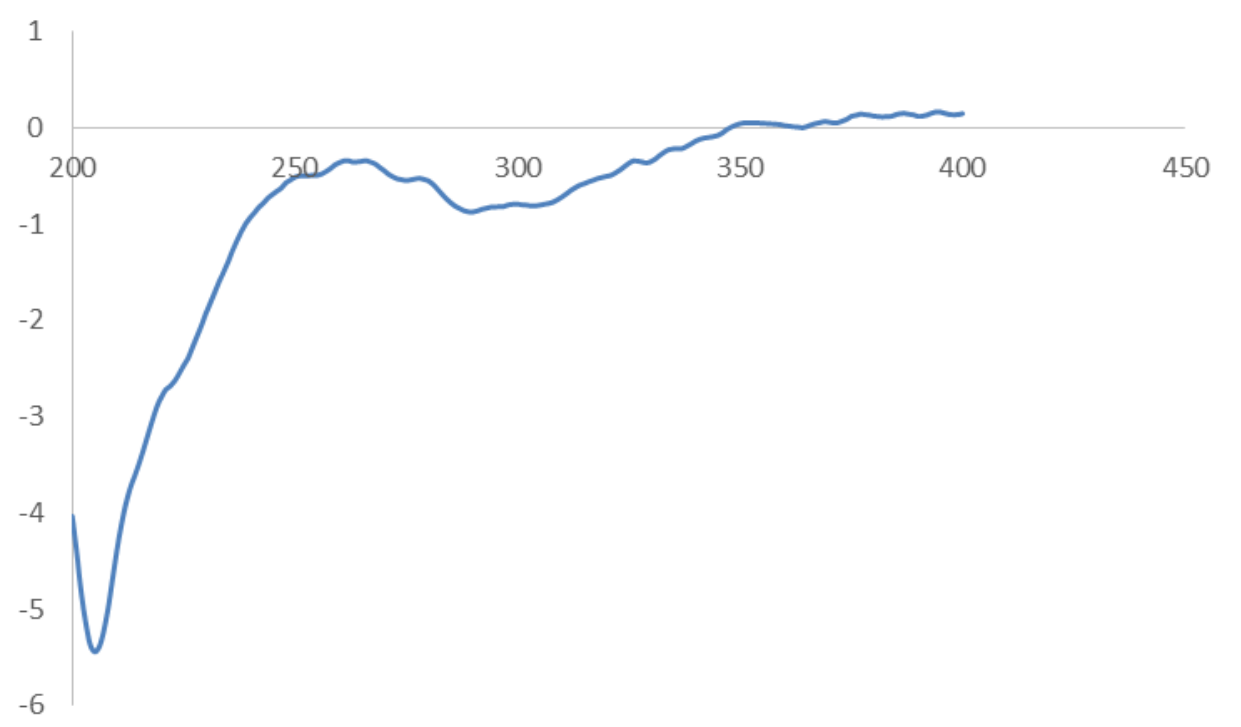

Figure S58. The CD spectra of 2. 
S1. ECD Calculation Details

\section{ECD calculation details}

\section{Methods}

Monte Carlo conformational searches were carried out by means of the Spartan's 10 software using Merck Molecular Force Field (MMFF). The conformers with Boltzmann-population of over 5\% were chosen for ECD calculations, and then the conformers were initially optimized at B3LYP/6-31g (d, p) level in $\mathrm{MeOH}$ using the CPCM polarizable conductor calculation model. The theoretical calculation of ECD was conducted in $\mathrm{MeOH}$ using Time-dependent Density functional theory (TD-DFT) at the B3LYP/6-31+g (d, p) level for all conformers of compounds 1-6R. Rotatory strengths for a total of 30 excited states were calculated. ECD spectra were generated using the program SpecDis 1.6 (University of Würzburg, Würzburg, Germany) and GraphPad Prism 5 (University of California San Diego, USA) from dipole-length rotational strengths by applying Gaussian band shapes with sigma $=0.3 \mathrm{eV}$.

\section{Results}

Table S1.2.1. Gibbs free energies ${ }^{a}$ and equilibrium populations ${ }^{b}$ of low-energy conformers of 1-6R.

\begin{tabular}{|c|l|l|}
\hline \multirow{2}{*}{ Conformers } & \multicolumn{2}{|c|}{ In $\mathrm{MeOH}$} \\
\cline { 2 - 3 } & \multicolumn{1}{|c|}{$\Delta G$} & \multicolumn{1}{|c|}{$P(\%) / 100$} \\
\hline $\mathbf{1 - 6} \boldsymbol{R}-\mathbf{1}$ & 0.00 & 0.421 \\
\hline $\mathbf{1 - 6} \boldsymbol{R}-\mathbf{2}$ & 0.66 & 0.137 \\
\hline $\mathbf{1 - 6} \boldsymbol{R}-\mathbf{3}$ & 0.81 & 0.108 \\
\hline $\mathbf{1 - 6} \boldsymbol{R}-\mathbf{4}$ & 0.95 & 0.085 \\
\hline $\mathbf{1 - 6} \boldsymbol{R}-\mathbf{5}$ & 1.12 & 0.064 \\
\hline
\end{tabular}

${ }^{a} \mathrm{~B} 3 \mathrm{LYP} / 6-31+\mathrm{G}(\mathrm{d}, \mathrm{p})$, in $\mathrm{kcal} / \mathrm{mol} .{ }^{b}$ From $\Delta G$ values at $298.15 \mathrm{~K}$.

Table S1.2.2. Cartesian coordinates for the low-energy reoptimized MMFF conformers of 1-6R at B3LYP/6-311+G(d,p) level of theory in $\mathrm{CH}_{3} \mathrm{OH}$.

\begin{tabular}{|l|l|l|l|l|c|}
\hline \multicolumn{2}{|c|}{ 1-6R-1 } & \multicolumn{4}{c|}{$\begin{array}{c}\text { Standard Orientation } \\
\text { (Ångstroms) }\end{array}$} \\
\hline $\begin{array}{c}\text { Center } \\
\text { number }\end{array}$ & $\begin{array}{c}\text { Atomic } \\
\text { number }\end{array}$ & $\begin{array}{c}\text { Atomic } \\
\text { Type }\end{array}$ & \multicolumn{1}{|c|}{ X } & \multicolumn{1}{c|}{ Y } & $\mathrm{Z}$ \\
\hline & & & - & - & \\
1. & 6. & 0. & 5.358832 & 1.700791 & 0.156550 \\
\hline & 6. & 0. & - & - & \\
\hline 3. & 6. & 0. & 5.752169 & 1.340209 & 1.575752 \\
\hline & 6. & 0. & - & - & \\
\hline 4. & 6.306707 & 0.018612 & 2.057060 \\
\hline
\end{tabular}




\begin{tabular}{|c|c|c|c|c|c|}
\hline 5. & 6. & 0. & $\begin{array}{l}- \\
3.994338\end{array}$ & 0.417086 & $\begin{array}{l}- \\
0.055929\end{array}$ \\
\hline 6. & 6. & 0. & $\begin{array}{l}- \\
3.981687\end{array}$ & $\begin{array}{l}- \\
1.143726\end{array}$ & $\begin{array}{l}- \\
0.269919\end{array}$ \\
\hline 7. & 6. & 0. & $\begin{array}{l}- \\
4.118375\end{array}$ & 2.153787 & 1.887129 \\
\hline 8. & 6. & 0. & $\begin{array}{l} \\
2.618352\end{array}$ & 1.007722 & $-\overline{0}-300118$ \\
\hline 9. & 6. & 0. & $\begin{array}{l} \\
2.282148\end{array}$ & 1.739314 & $\begin{array}{l}- \\
1.361456\end{array}$ \\
\hline 10. & 6. & 0. & $\begin{array}{l}- \\
0.913826\end{array}$ & 2.317572 & $-\overline{1.618143}$ \\
\hline 11. & 6. & 0. & $\begin{array}{l}- \\
0.945271\end{array}$ & 3.842779 & 1.744935 \\
\hline 12. & 8. & 0. & $\begin{array}{l}- \\
0.002058\end{array}$ & 1.981618 & $\begin{array}{l}- \\
0.551298\end{array}$ \\
\hline 13. & 6. & 0. & $-\overline{3.745737}$ & $\begin{array}{l}- \\
1.479139\end{array}$ & $\begin{array}{l}- \\
1.753693\end{array}$ \\
\hline 14. & 6. & 0. & $\begin{array}{l}- \\
2.869468 \\
\end{array}$ & $\begin{array}{l}- \\
1.810480\end{array}$ & 0.568409 \\
\hline 15. & 8. & 0. & $-\overline{6.435067}$ & $\begin{array}{l}- \\
2.075396 \\
\end{array}$ & 2.275577 \\
\hline 16. & 8. & 0. & $-\overline{4.948145}$ & 0.926496 & $\begin{array}{l} \\
1.006949\end{array}$ \\
\hline 17. & 6. & 0. & 1.050885 & 1.138900 & $\begin{array}{l}- \\
0.872321\end{array}$ \\
\hline 18. & 8. & 0. & 0.568811 & $\begin{array}{l}- \\
0.200189\end{array}$ & $\begin{array}{l}- \\
0.988625\end{array}$ \\
\hline 19. & 6. & 0. & 1.597614 & $\begin{array}{l} \\
1.111461\end{array}$ & $\begin{array}{l}- \\
1.355593\end{array}$ \\
\hline 20. & 6. & 0. & 2.694865 & $\begin{array}{l} \\
1.144441\end{array}$ & $\begin{array}{l}- \\
0.272106\end{array}$ \\
\hline 21. & 6. & 0. & 3.217093 & 0.264468 & 0.027962 \\
\hline 22. & 6. & 0. & 2.078111 & 1.252281 & 0.262639 \\
\hline 23. & 8. & 0. & 2.623686 & 2.551375 & 0.303288 \\
\hline 24. & 8. & 0. & 3.804611 & $\begin{array}{l}- \\
1.935279\end{array}$ & $\begin{array}{l}- \\
0.708255\end{array}$ \\
\hline 25. & 8. & 0. & 4.012200 & 0.240430 & 1.234826 \\
\hline 26. & 16. & 0. & 5.644716 & 0.048720 & 1.109657 \\
\hline 27. & 8. & 0. & 6.077937 & 0.615188 & $-\overline{0.158685}$ \\
\hline 28. & 8. & 0. & 6.182204 & 0.482137 & 2.376314 \\
\hline 29. & 8. & 0. & 5.758102 & - & 1.068382 \\
\hline
\end{tabular}




\begin{tabular}{|c|c|c|c|c|c|}
\hline & & & & 1.550854 & \\
\hline 30. & 6. & 0. & 0.904220 & $\begin{array}{l}- \\
2.461028\end{array}$ & $\begin{array}{l}- \\
1.534508\end{array}$ \\
\hline 31. & 8. & 0. & 1.902282 & $\begin{array}{l}- \\
3.425076\end{array}$ & $\begin{array}{l} \\
1.887107\end{array}$ \\
\hline 32. & 1. & 0. & $\begin{array}{l}- \\
6.129169\end{array}$ & $\begin{array}{l}- \\
1.282808\end{array}$ & $\begin{array}{l}- \\
0.503890\end{array}$ \\
\hline 33. & 1. & 0. & $\begin{array}{l}- \\
5.393557\end{array}$ & $\begin{array}{l} \\
\end{array} .789683$ & 0.054672 \\
\hline 34. & 1. & 0. & $\begin{array}{l}- \\
5.681179\end{array}$ & 0.272525 & 3.035730 \\
\hline 35. & 1. & 0. & $\begin{array}{l}- \\
4.331800\end{array}$ & 2.948157 & 1.160235 \\
\hline 36. & 1. & 0. & $\begin{array}{l}- \\
4.661250\end{array}$ & 2.382209 & 2.807048 \\
\hline 37. & 1. & 0. & $\begin{array}{l}- \\
3.044363\end{array}$ & 2.217985 & 2.094403 \\
\hline 38. & 1. & 0. & $\begin{array}{l}- \\
1.858331\end{array}$ & 0.793978 & 0.444869 \\
\hline 39. & 1. & 0. & $\begin{array}{l}- \\
3.025548\end{array}$ & 1.952707 & $\begin{array}{l}- \\
2.127288\end{array}$ \\
\hline 40. & 1. & 0. & $\begin{array}{l}- \\
0.525855\end{array}$ & 1.891216 & $\begin{array}{l} \\
2.555094 \\
\end{array}$ \\
\hline 41. & 1. & 0. & 0.048784 & 4.228251 & $\begin{array}{l} \\
1.989267 \\
\end{array}$ \\
\hline 42. & 1. & 0. & $\begin{array}{l}- \\
1.274167\end{array}$ & 4.290118 & $\begin{array}{l} \\
0.802402 \\
\end{array}$ \\
\hline 43. & 1. & 0. & $\begin{array}{l}- \\
1.638624\end{array}$ & 4.151710 & $\begin{array}{l} \\
2.533185 \\
\end{array}$ \\
\hline 44. & 1. & 0. & $\begin{array}{l}- \\
4.514022\end{array}$ & $\begin{array}{l} \\
1.034984\end{array}$ & $\begin{array}{l}- \\
2.389572 \\
\end{array}$ \\
\hline 45. & 1. & 0. & $\begin{array}{l}- \\
3.771522\end{array}$ & $\begin{array}{l}- \\
2.565670\end{array}$ & $\begin{array}{l}- \\
1.891426 \\
\end{array}$ \\
\hline 46. & 1. & 0. & $\begin{array}{l}- \\
2.769716\end{array}$ & $\begin{array}{l}- \\
1.117767\end{array}$ & $\begin{array}{l}- \\
2.090884\end{array}$ \\
\hline 47. & 1. & 0. & $\begin{array}{l}- \\
1.876181\end{array}$ & $\begin{array}{l}- \\
1.508817\end{array}$ & 0.223744 \\
\hline 48. & 1. & 0. & $\begin{array}{l}- \\
2.946958\end{array}$ & $\begin{array}{l}- \\
1.572829\end{array}$ & 1.633314 \\
\hline 49. & 1. & 0. & $\begin{array}{l}- \\
2.943070\end{array}$ & $\begin{array}{l}- \\
2.898520\end{array}$ & 0.467663 \\
\hline 50. & 1. & 0. & $\begin{array}{l} \\
5.056474\end{array}$ & 1.872723 & $\begin{array}{l} \\
0.839748 \\
\end{array}$ \\
\hline
\end{tabular}




\begin{tabular}{|c|c|c|c|c|c|}
\hline 51. & 1. & 0. & 1.519064 & 1.435006 & \begin{tabular}{|l|}
- \\
1.828862 \\
\end{tabular} \\
\hline 52. & 1. & 0. & 2.064687 & $\begin{array}{l}- \\
0.814389\end{array}$ & $\begin{array}{l}- \\
2.308822 \\
\end{array}$ \\
\hline 53. & 1. & 0. & 2.264216 & 1.556757 & 0.652941 \\
\hline 54. & 1. & 0. & 3.837662 & 0.617300 & $\begin{array}{l}- \\
0.802442\end{array}$ \\
\hline 55. & 1. & 0. & 1.562626 & 0.990911 & 1.199340 \\
\hline 56. & 1. & 0. & 1.887644 & 3.154039 & \begin{tabular}{|l|l|}
0.478458 \\
\end{tabular} \\
\hline 57. & 1. & 0. & 3.439109 & $\begin{array}{l}- \\
2.764199\end{array}$ & $\begin{array}{l}- \\
1.072415\end{array}$ \\
\hline 58. & 1. & 0. & 5.149522 & $\begin{array}{l}- \\
1.871174\end{array}$ & 0.346715 \\
\hline 59. & 1. & 0. & 0.404057 & $\begin{array}{l} \\
2.729122\end{array}$ & $\begin{array}{l} \\
0.594103\end{array}$ \\
\hline 60. & 1. & 0. & 0.141990 & $\begin{array}{l}- \\
2.372536\end{array}$ & $\begin{array}{l}- \\
2.318322\end{array}$ \\
\hline 61. & 1. & 0. & 1.509399 & - & $\begin{array}{l}- \\
1.871560\end{array}$ \\
\hline & & & & & \\
\hline
\end{tabular}

\begin{tabular}{|c|c|c|c|c|c|}
\hline \multicolumn{2}{|c|}{$1-6 R-2$} & \multicolumn{4}{|c|}{$\begin{array}{l}\text { Standard Orientation } \\
\text { (Angstroms) }\end{array}$} \\
\hline $\begin{array}{l}\text { Center } \\
\text { number }\end{array}$ & $\begin{array}{l}\text { Atom } \\
\text { number }\end{array}$ & Type & $\mathrm{X}$ & Y & Z \\
\hline 1. & 6. & 0. & 5.451362 & 1.320381 & 0.487835 \\
\hline 2. & 6. & 0. & 5.727320 & 0.720419 & 1.851839 \\
\hline 3. & 6. & 0. & 5.123525 & 0.601117 & 2.111197 \\
\hline 4. & 6. & 0. & 4.269993 & 1.208305 & 1.266951 \\
\hline 5. & 6. & 0. & 3.868233 & $-\overline{0.569577}$ & $-\overline{0.081302}$ \\
\hline 6. & 6. & 0. & 4.044189 & 0.996414 & $\begin{array}{l}- \\
0.064678\end{array}$ \\
\hline 7. & 6. & 0. & 3.730578 & $\begin{array}{l}- \\
2.582573 \\
\end{array}$ & 1.573008 \\
\hline 8. & 6. & 0. & 2.441681 & $-\overline{0.953776}$ & $\begin{array}{l}- \\
0.432325\end{array}$ \\
\hline 9. & 6. & 0. & 2.059989 & - & - \\
\hline
\end{tabular}




\begin{tabular}{|c|c|c|c|c|c|}
\hline & & & & 1.471548 & 1.599254 \\
\hline 10. & 6. & 0. & 0.648891 & $\begin{array}{l}- \\
1.859089\end{array}$ & $\begin{array}{l} \\
1.962233\end{array}$ \\
\hline 11. & 6. & 0. & 0.541804 & $\begin{array}{l}- \\
3.331390\end{array}$ & $\begin{array}{l}- \\
2.366310\end{array}$ \\
\hline 12. & 8. & 0. & $\begin{array}{l}- \\
0.252506\end{array}$ & $\begin{array}{l} \\
1.640152\end{array}$ & $\begin{array}{l} \\
0.853173 \\
\end{array}$ \\
\hline 13. & 6. & 0. & 3.933128 & 1.563856 & $\begin{array}{l}- \\
1.491089\end{array}$ \\
\hline 14. & 6. & 0. & 2.971510 & 1.666243 & \begin{tabular}{|l|}
0.820837 \\
\end{tabular} \\
\hline 15. & 8. & 0. & 6.444276 & 1.264864 & 2.679725 \\
\hline 16. & 8. & 0. & 4.788444 & $\begin{array}{l} \\
1.048141\end{array}$ & $\begin{array}{l} \\
1.079736 \\
\end{array}$ \\
\hline 17. & 6. & 0. & $\begin{array}{l} \\
1.208196\end{array}$ & $\begin{array}{l}- \\
0.647078\end{array}$ & $\begin{array}{l} \\
1.016518 \\
\end{array}$ \\
\hline 18. & 8. & 0. & $\begin{array}{l}- \\
0.616041\end{array}$ & 0.635938 & $\begin{array}{l}- \\
0.888408\end{array}$ \\
\hline 19. & 6. & 0. & $-\overline{1.556677}$ & 1.703570 & - \\
\hline 20. & 6. & 0. & $\begin{array}{l}- \\
2.635588\end{array}$ & 1.674806 & $\begin{array}{l} \\
0.007031\end{array}$ \\
\hline 21. & 6. & 0. & $\begin{array}{l}- \\
3.267546\end{array}$ & 0.282635 & 0.048588 \\
\hline 22. & 6. & 0. & $\begin{array}{l}- \\
2.234604\end{array}$ & $\begin{array}{l}- \\
0.832499\end{array}$ & 0.103125 \\
\hline 23. & 8. & 0. & $\begin{array}{l}- \\
2.925221\end{array}$ & $\begin{array}{l}- \\
2.075961\end{array}$ & $\begin{array}{l} \\
0.024903\end{array}$ \\
\hline 24. & 8. & 0. & $\begin{array}{l}- \\
3.591464\end{array}$ & 2.660853 & $\begin{array}{l} \\
0.330976 \\
\end{array}$ \\
\hline 25. & 8. & 0. & $\begin{array}{l} \\
4.066928 \\
\end{array}$ & 0.188474 & 1.262000 \\
\hline 26. & 16. & 0. & $\begin{array}{l} \\
5.653845\end{array}$ & $\begin{array}{l}- \\
0.208624\end{array}$ & 1.122950 \\
\hline 27. & 8. & 0. & $\begin{array}{l}- \\
6.073392\end{array}$ & $\begin{array}{l}- \\
0.506281\end{array}$ & 2.474003 \\
\hline 28. & 8. & 0. & $\begin{array}{l}- \\
6.322356 \\
\end{array}$ & 0.798845 & 0.316936 \\
\hline 29. & 8. & 0. & $\begin{array}{l}- \\
5.563265\end{array}$ & $\begin{array}{l}- \\
1.541678\end{array}$ & 0.225737 \\
\hline 30. & 6. & 0. & $\begin{array}{l}-747560 \\
0.74\end{array}$ & 2.995092 & $\begin{array}{l}-118881 \\
\end{array}$ \\
\hline 31. & 8. & 0. & 0.218460 & 2.986149 & 2.151022 \\
\hline
\end{tabular}




\begin{tabular}{|c|c|c|c|c|c|}
\hline 32. & 1. & 0. & 6.202907 & 0.908467 & $\begin{array}{l}- \\
0.197811\end{array}$ \\
\hline 33. & 1. & 0. & 5.613861 & 2.400743 & 0.544643 \\
\hline 34. & 1. & 0. & 5.419885 & $\begin{array}{l}- \\
1.075231\end{array}$ & 3.044110 \\
\hline 35. & 1. & 0. & 4.212967 & $\begin{array}{l}- \\
3.006314\end{array}$ & 2.456612 \\
\hline 36. & 1. & 0. & 2.648835 & $\begin{array}{l}- \\
2.561806\end{array}$ & 1.746758 \\
\hline 37. & 1. & 0. & 3.887493 & $\begin{array}{l}- \\
3.275076\end{array}$ & 0.735744 \\
\hline 38. & 1. & 0. & 1.690333 & $\begin{array}{l}- \\
0.777133\end{array}$ & 0.331141 \\
\hline 39. & 1. & 0. & 2.800344 & $\begin{array}{l}- \\
1.641876\end{array}$ & $\begin{array}{l} \\
2.378653\end{array}$ \\
\hline 40. & 1. & 0. & 0.318422 & $\begin{array}{l} \\
1.234116\end{array}$ & $\begin{array}{l}- \\
2.804929 \\
\end{array}$ \\
\hline 41. & 1. & 0. & $\begin{array}{l}- \\
0.477939\end{array}$ & $\begin{array}{l} \\
3.571331\end{array}$ & $\begin{array}{l} \\
2.681519\end{array}$ \\
\hline 42. & 1. & 0. & 0.810250 & $\begin{array}{l}- \\
3.972617\end{array}$ & $\begin{array}{l} \\
1.521538 \\
\end{array}$ \\
\hline 43. & 1. & 0. & 1.219276 & $\begin{array}{l} \\
3.554489\end{array}$ & $\begin{array}{l} \\
3.195948 \\
\end{array}$ \\
\hline 44. & 1. & 0. & 4.701304 & 1.150404 & $\begin{array}{l}- \\
2.147243 \\
\end{array}$ \\
\hline 45. & 1. & 0. & 4.048239 & 2.652130 & $\begin{array}{l}- \\
1.465110\end{array}$ \\
\hline 46. & 1. & 0. & 2.957458 & 1.339743 & $\begin{array}{l}- \\
1.932800\end{array}$ \\
\hline 47. & 1. & 0. & 3.173141 & 2.739917 & 0.887639 \\
\hline 48. & 1. & 0. & 1.969526 & 1.543736 & 0.399525 \\
\hline 49. & 1. & 0. & 2.961666 & 1.267632 & 1.839573 \\
\hline 50. & 1. & 0. & 4.797444 & $\begin{array}{l}- \\
2.014193\end{array}$ & $\begin{array}{l}- \\
1.037646\end{array}$ \\
\hline 51. & 1. & 0. & $\begin{array}{l}- \\
1.700343\end{array}$ & $\begin{array}{l}- \\
0.727445\end{array}$ & $\begin{array}{l}- \\
2.003297\end{array}$ \\
\hline 52. & 1. & 0. & $\begin{array}{l}- \\
2.041241\end{array}$ & 1.575980 & $\begin{array}{l} \\
2.083231\end{array}$ \\
\hline 53. & 1. & 0. & $\begin{array}{l}- \\
2.158412\end{array}$ & 1.873436 & 0.966317 \\
\hline 54. & 1. & 0. & $\begin{array}{l}- \\
3.910908\end{array}$ & 0.158000 & $\begin{array}{l}- \\
0.829119 \\
\end{array}$ \\
\hline 55. & 1. & 0. & - & - & 1.058889 \\
\hline
\end{tabular}




\begin{tabular}{|l|l|l|l|l|l|}
\hline & & & 1.697238 & 0.782933 & \\
\hline 56. & 1. & 0. & -2.273698 & 2.786973 & 0.051609 \\
\hline 57. & 1. & 0. & -4.384921 & 2.519844 & 0.207270 \\
\hline 58. & 1. & 0. & -4.670497 & 1.962345 & 0.319721 \\
\hline 59. & 1. & 0. & -1.422072 & 3.833655 & 1.304853 \\
\hline 60. & 1. & 0. & -0.288514 & 3.138268 & 0.127288 \\
\hline 61. & 1. & 0. & 0.761700 & 2.197854 & 2.010968 \\
\hline & & & & & \\
\hline & & & & & \\
\hline
\end{tabular}

\begin{tabular}{|c|c|c|c|c|c|}
\hline \multicolumn{2}{|c|}{$1-6 R-3$} & \multicolumn{4}{|c|}{$\begin{array}{l}\text { Standard Orientation } \\
\text { (Ångstroms) }\end{array}$} \\
\hline $\begin{array}{l}\text { Center } \\
\text { number }\end{array}$ & $\begin{array}{l}\text { Atom } \\
\text { number }\end{array}$ & Type & $\mathrm{X}$ & Y & Z \\
\hline 1. & 6. & 0. & 2.139633 & $\overline{1.545451}$ & 0.310336 \\
\hline 2. & 6. & 0. & 2.260087 & $\begin{array}{l}- \\
1.482285\end{array}$ & $\begin{array}{l}- \\
1.196273\end{array}$ \\
\hline 3. & 6. & 0. & 3.275055 & $\overline{0} .612640$ & $-\overline{1.783120}$ \\
\hline 4. & 6. & 0. & 3.880905 & 0.354446 & $-\overline{1.061232}$ \\
\hline 5. & 6. & 0. & 3.511710 & 0.573321 & 0.410216 \\
\hline 6. & 6. & 0. & 3.247818 & $\begin{array}{l}- \\
0.817589\end{array}$ & 1.102727 \\
\hline 7. & 6. & 0. & 4.878173 & 1.292090 & $-\overline{1.671598}$ \\
\hline 8. & 6. & 0. & 2.306795 & 1.503497 & 0.529439 \\
\hline 9. & 6. & 0. & 1.474710 & 1.873079 & $\begin{array}{l}- \\
0.446912\end{array}$ \\
\hline 10. & 6. & 0. & 0.236236 & 2.714913 & \begin{tabular}{|l} 
\\
0.278129 \\
\end{tabular} \\
\hline 11. & 6. & 0. & 0.305389 & 4.011476 & $\begin{array}{l}- \\
1.090504\end{array}$ \\
\hline 12. & 8. & 0. & $\begin{array}{l}- \\
0.814144\end{array}$ & 1.848221 & $\begin{array}{l} \\
0.754112\end{array}$ \\
\hline
\end{tabular}




\begin{tabular}{|c|c|c|c|c|c|}
\hline 13. & 6. & 0. & 4.550769 & 1.649575 & 1.108395 \\
\hline 14. & 6. & 0. & 2.792488 & 0.640928 & 2.564199 \\
\hline 15. & 8. & 0. & 1.504454 & $\begin{array}{l}- \\
2.131582\end{array}$ & $\begin{array}{l} \\
1.934787\end{array}$ \\
\hline 16. & 8. & 0. & 4.612794 & 1.163373 & 1.118054 \\
\hline 17. & 6. & 0. & $\begin{array}{l}- \\
2.147879\end{array}$ & 2.194007 & $\begin{array}{l}- \\
0.450809\end{array}$ \\
\hline 18. & 8. & 0. & $\begin{array}{l}- \\
2.426999\end{array}$ & 2.226079 & 0.938361 \\
\hline 19. & 6. & 0. & $\begin{array}{l}- \\
2.500130\end{array}$ & 0.974721 & 1.642170 \\
\hline 20. & 6. & 0. & $\begin{array}{l}- \\
3.417065\end{array}$ & $\begin{array}{l} \\
0.036597\end{array}$ & 0.941114 \\
\hline 21. & 6. & 0. & $\begin{array}{l}- \\
3.134846 \\
\end{array}$ & $\begin{array}{l}- \\
0.171527\end{array}$ & $\begin{array}{l} \\
0.566651\end{array}$ \\
\hline 22. & 6. & 0. & $\begin{array}{l}- \\
3.050456\end{array}$ & 1.216394 & $\begin{array}{l} \\
1.232172 \\
\end{array}$ \\
\hline 23. & 8. & 0. & $\begin{array}{l} \\
4.348574\end{array}$ & 1.757547 & $\begin{array}{l} \\
1.372035 \\
\end{array}$ \\
\hline 24. & 8. & 0. & $\begin{array}{l}- \\
4.788180\end{array}$ & 0.387999 & 0.993712 \\
\hline 25. & 8. & 0. & $\begin{array}{l} \\
1.878303\end{array}$ & $\begin{array}{l}- \\
0.858011\end{array}$ & $\begin{array}{l} \\
0.820997 \\
\end{array}$ \\
\hline 26. & 16. & 0. & $\begin{array}{l} \\
1.800914\end{array}$ & $\begin{array}{l}- \\
2.469845\end{array}$ & $\begin{array}{l} \\
0.526903 \\
\end{array}$ \\
\hline 27. & 8. & 0. & $\begin{array}{l}- \\
1.045286\end{array}$ & $\begin{array}{l}- \\
2.624244\end{array}$ & 0.722994 \\
\hline 28. & 8. & 0. & $\begin{array}{l} \\
3.121182\end{array}$ & $\begin{array}{l}- \\
3.053721\end{array}$ & $\begin{array}{l}- \\
0.654482\end{array}$ \\
\hline 29. & 8. & 0. & $\begin{array}{l}- \\
0.920028\end{array}$ & $\begin{array}{l}- \\
2.930241\end{array}$ & $\begin{array}{l} \\
1.741268 \\
\end{array}$ \\
\hline 30. & 6. & 0. & $\begin{array}{l}- \\
1.099830\end{array}$ & 0.437522 & 1.994568 \\
\hline 31. & 8. & 0. & $\begin{array}{l}-171262 \\
\end{array}$ & $\begin{array}{l}- \\
0.679176\end{array}$ & 2.862883 \\
\hline 32. & 1. & 0. & 2.092488 & $\begin{array}{l}- \\
2.603788 \\
\end{array}$ & 0.591895 \\
\hline 33. & 1. & 0. & 1.154795 & $-\overline{1.140259}$ & 0.564998 \\
\hline 34. & 1. & 0. & 3.460653 & $-\overline{0.733486}$ & $\begin{array}{l}- \\
2.846933\end{array}$ \\
\hline
\end{tabular}




\begin{tabular}{|c|c|c|c|c|c|}
\hline 35. & 1. & 0. & 4.539116 & 2.332159 & $\begin{array}{l}- \\
1.582671\end{array}$ \\
\hline 36. & 1. & 0. & 5.831751 & 1.223707 & $\begin{array}{l}- \\
1.138238\end{array}$ \\
\hline 37. & 1. & 0. & 5.040349 & 1.073594 & $\begin{array}{l}- \\
2.728927\end{array}$ \\
\hline 38. & 1. & 0. & \begin{tabular}{|l|l|}
2.138402 \\
\end{tabular} & 1.862342 & 1.544013 \\
\hline 39. & 1. & 0. & 1.618894 & 1.510880 & $\begin{array}{l}- \\
1.462697\end{array}$ \\
\hline 40. & 1. & 0. & 0.068674 & 2.946296 & 0.780559 \\
\hline 41. & 1. & 0. & $\begin{array}{l} \\
0.615506 \\
\end{array}$ & 4.593243 & $\begin{array}{l} \\
0.992661\end{array}$ \\
\hline 42. & 1. & 0. & 0.463013 & 3.790939 & $\overline{2.150742}$ \\
\hline 43. & 1. & 0. & 1.132027 & 4.635537 & $\begin{array}{l}- \\
0.736510\end{array}$ \\
\hline 44. & 1. & 0. & 4.920603 & $\begin{array}{l} \\
1.860082\end{array}$ & 0.101606 \\
\hline 45. & 1. & 0. & 4.368069 & $\begin{array}{l}- \\
2.608094\end{array}$ & 1.604341 \\
\hline 46. & 1. & 0. & 5.337102 & $\begin{array}{l}- \\
1.126409\end{array}$ & 1.656323 \\
\hline 47. & 1. & 0. & 2.737273 & $\begin{array}{l}- \\
1.619660\end{array}$ & 3.051299 \\
\hline 48. & 1. & 0. & 3.508728 & $\begin{array}{l}- \\
0.028033\end{array}$ & 3.116969 \\
\hline 49. & 1. & 0. & 1.799973 & $\begin{array}{l}- \\
0.190960\end{array}$ & 2.647312 \\
\hline 50. & 1. & 0. & \begin{tabular}{|l}
4.587417 \\
\end{tabular} & 2.117904 & 0.970173 \\
\hline 51. & 1. & 0. & $\begin{array}{l}- \\
2.383867\end{array}$ & 3.210606 & $\begin{array}{l} \\
0.785591\end{array}$ \\
\hline 52. & 1. & 0. & $\begin{array}{l}- \\
2.980562 \\
\end{array}$ & 1.230473 & 2.596321 \\
\hline 53. & 1. & 0. & $\begin{array}{l} \\
3.309047 \\
\end{array}$ & $\begin{array}{l} \\
1.007234 \\
\end{array}$ & 1.439057 \\
\hline 54. & 1. & 0. & $\begin{array}{l} \\
.948496 \\
\end{array}$ & $\begin{array}{l} \\
0.732996\end{array}$ & $\begin{array}{l}- \\
1.030875\end{array}$ \\
\hline 55. & 1. & 0. & $\begin{array}{l}- \\
2.629092 \\
\end{array}$ & 1.104441 & $\begin{array}{l} \\
2.234539 \\
\end{array}$ \\
\hline 56. & 1. & 0. & 4.794266 & 1.640394 & $\begin{array}{l} \\
0.515169\end{array}$ \\
\hline 57. & 1. & 0. & $\begin{array}{l}- \\
5.012226\end{array}$ & 0.580841 & 1.913871 \\
\hline
\end{tabular}




\begin{tabular}{|c|c|c|c|c|c|}
\hline 58. & 1. & 0. & 0.062756 & $\begin{array}{l} \\
2.650328 \\
\end{array}$ & \begin{tabular}{|l|}
- \\
1.674867 \\
\end{tabular} \\
\hline 59. & 1. & 0. & $\begin{array}{l}- \\
0.545911 \\
\end{array}$ & 0.216633 & 1.080425 \\
\hline 60. & 1. & 0. & $\begin{array}{l}- \\
0.573676 \\
\end{array}$ & 1.242364 & 2.521167 \\
\hline 61. & 1. & 0. & \begin{tabular}{|l|} 
\\
1.138497 \\
\end{tabular} & $\begin{array}{l}- \\
1.476694\end{array}$ & 2.309350 \\
\hline & & & & & \\
\hline
\end{tabular}

\begin{tabular}{|c|c|c|c|c|c|}
\hline \multicolumn{2}{|c|}{$1-6 R-4$} & \multicolumn{4}{|c|}{$\begin{array}{l}\text { Standard Orientation } \\
\text { (Angstroms) }\end{array}$} \\
\hline $\begin{array}{l}\text { Center } \\
\text { number }\end{array}$ & $\begin{array}{c}\text { Atom } \\
\text { number }\end{array}$ & Type & $\mathrm{X}$ & Y & Z \\
\hline 1. & 6. & 0. & $-\overline{6.945108}$ & $-\overline{0}-383751$ & $\begin{array}{l}- \\
0.307340\end{array}$ \\
\hline 2. & 6. & 0. & $\begin{array}{l}- \\
7.029356\end{array}$ & 0.992460 & $\begin{array}{l}- \\
0.936757\end{array}$ \\
\hline 3. & 6. & 0. & $-\overline{5.750219}$ & 1.577624 & $\begin{array}{l} \\
1.384462\end{array}$ \\
\hline 4. & 6. & 0. & $\begin{array}{l}- \\
4.550732\end{array}$ & 1.015357 & $\begin{array}{l} \\
1.148423\end{array}$ \\
\hline 5. & 6. & 0. & -4.420139 & $\begin{array}{l}- \\
0.328422\end{array}$ & $\begin{array}{l}- \\
0.395901\end{array}$ \\
\hline 6. & 6. & 0. & $\overline{5.664398}$ & $\overline{0} .606723$ & 0.528856 \\
\hline 7. & 6. & 0. & $\begin{array}{l}- \\
3.287472 \\
\end{array}$ & 1.635987 & $-\overline{1.688515}$ \\
\hline 8. & 6. & 0. & $\begin{array}{l}- \\
3.125887\end{array}$ & $\begin{array}{l}- \\
0.350723\end{array}$ & 0.398898 \\
\hline 9. & 6. & 0. & $\begin{array}{l}- \\
2.193109\end{array}$ & $-\overline{1.300069}$ & 0.319141 \\
\hline 10. & 6. & 0. & $\begin{array}{l}- \\
0.923055\end{array}$ & 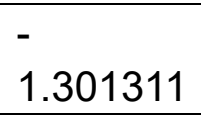 & 1.121659 \\
\hline 11. & 6. & 0. & $\begin{array}{l}- \\
0.795033\end{array}$ & $\begin{array}{l} \\
2.541414\end{array}$ & 2.007373 \\
\hline 12. & 8. & 0. & 0.170845 & $\begin{array}{l}- \\
1.270717\end{array}$ & 0.160433 \\
\hline 13. & 6. & 0. & $\begin{array}{l}- \\
5.643419\end{array}$ & $\begin{array}{l}- \\
2.061802\end{array}$ & 1.030587 \\
\hline 14. & 6. & 0. & - & 0.336860 & \begin{tabular}{|l|}
1.750865 \\
\end{tabular} \\
\hline
\end{tabular}




\begin{tabular}{|c|c|c|c|c|c|}
\hline & & & 5.675129 & & \\
\hline 15. & 8. & 0. & 8.090588 & 1.574813 & 1.111917 \\
\hline 16. & 8. & 0. & $\begin{array}{l}- \\
4.429210\end{array}$ & $\begin{array}{l}- \\
1.377537\end{array}$ & $\begin{array}{l} \\
1.379449\end{array}$ \\
\hline 17. & 6. & 0. & 1.327985 & $\begin{array}{l}- \\
0.643419\end{array}$ & 0.594001 \\
\hline 18. & 8. & 0. & 1.121583 & 0.771262 & 0.581438 \\
\hline 19. & 6. & 0. & 2.257746 & 1.483840 & 1.050493 \\
\hline 20. & 6. & 0. & 3.471087 & 1.220557 & 0.134665 \\
\hline 21. & 6. & 0. & 3.724842 & $\begin{array}{l}- \\
0.281912\end{array}$ & $\begin{array}{l} \\
0.027258\end{array}$ \\
\hline 22. & 6. & 0. & 2.446475 & $-\overline{1.036853}$ & $\begin{array}{l} \\
0.379941 \\
\end{array}$ \\
\hline 23. & 8. & 0. & 2.712190 & $\begin{array}{l}- \\
2.418272\end{array}$ & $\begin{array}{l}- \\
0.291608\end{array}$ \\
\hline 24. & 8. & 0. & 4.652469 & 1.804756 & \begin{tabular}{|l|l|}
0.691448 \\
\end{tabular} \\
\hline 25. & 8. & 0. & 4.659908 & 0.501629 & $\begin{array}{l}- \\
1.108044 \\
\end{array}$ \\
\hline 26. & 16. & 0. & 6.265078 & $\begin{array}{l}- \\
0.632275\end{array}$ & $\begin{array}{l} \\
0.759598 \\
\end{array}$ \\
\hline 27. & 8. & 0. & 6.402530 & $\begin{array}{l}- \\
1.183901\end{array}$ & 0.579783 \\
\hline 28. & 8. & 0. & 6.862398 & $\begin{array}{l} \\
1.250769\end{array}$ & $\begin{array}{l} \\
1.918053 \\
\end{array}$ \\
\hline 29. & 8. & 0. & 6.702355 & 0.911560 & $\begin{array}{l}- \\
0.768344\end{array}$ \\
\hline 30. & 6. & 0. & 1.836679 & 2.952688 & 1.063208 \\
\hline 31. & 8. & 0. & 2.957655 & 3.732412 & 1.492624 \\
\hline 32. & 1. & 0. & $\begin{array}{l}- \\
6.963260\end{array}$ & $\begin{array}{l} \\
1.111852\end{array}$ & $\begin{array}{l}-128324 \\
1.128\end{array}$ \\
\hline 33. & 1. & 0. & $\begin{array}{l}- \\
7.843148\end{array}$ & $\begin{array}{l}- \\
0.545145\end{array}$ & 0.296395 \\
\hline 34. & 1. & 0. & $\begin{array}{l}- \\
5.831524\end{array}$ & 2.508286 & $\begin{array}{l} \\
1.941465 \\
\end{array}$ \\
\hline 35. & 1. & 0. & $\begin{array}{l}- \\
2.698535\end{array}$ & 0.914066 & $\begin{array}{l}- \\
2.268760\end{array}$ \\
\hline 36. & 1. & 0. & $\begin{array}{l}- \\
3.514186\end{array}$ & 2.485698 & $\begin{array}{l}- \\
2.336367\end{array}$ \\
\hline 37. & 1. & 0. & $\begin{array}{l}- \\
2.626685\end{array}$ & 1.978652 & $\begin{array}{l} \\
0.884408\end{array}$ \\
\hline 38. & 1. & 0. & $\begin{array}{l}- \\
2.969791\end{array}$ & 0.490994 & 1.071661 \\
\hline
\end{tabular}




\begin{tabular}{|c|c|c|c|c|c|}
\hline 39. & 1. & 0. & \begin{tabular}{|l|} 
\\
2.338881 \\
\end{tabular} & $\begin{array}{l} \\
2.147707\end{array}$ & \begin{tabular}{|l|}
- \\
0.347699 \\
\end{tabular} \\
\hline 40. & 1. & 0. & $\begin{array}{l}- \\
0.872644 \\
\end{array}$ & $\begin{array}{l}- \\
0.389040\end{array}$ & 1.726999 \\
\hline 41. & 1. & 0. & 0.162679 & 2.555700 & 2.536772 \\
\hline 42. & 1. & 0. & $\begin{array}{l}- \\
0.866271\end{array}$ & $\begin{array}{l}- \\
3.450709\end{array}$ & 1.402710 \\
\hline 43. & 1. & 0. & $\begin{array}{l} \\
1.595808\end{array}$ & $\begin{array}{l}- \\
2.560255\end{array}$ & 2.752678 \\
\hline 44. & 1. & 0. & $\begin{array}{l}- \\
5.661701\end{array}$ & $\begin{array}{l}- \\
2.770175\end{array}$ & 0.200337 \\
\hline 45. & 1. & 0. & $\begin{array}{l}- \\
6.520450\end{array}$ & $\begin{array}{l}- \\
2.244894\end{array}$ & 1.660288 \\
\hline 46. & 1. & 0. & $\begin{array}{l}- \\
4.751480\end{array}$ & $\begin{array}{l}- \\
2.262230\end{array}$ & 1.631363 \\
\hline 47. & 1. & 0. & $\begin{array}{l}- \\
4.848737\end{array}$ & 0.118276 & 2.433234 \\
\hline 48. & 1. & 0. & $\begin{array}{l}- \\
5.615979 \\
\end{array}$ & 1.391948 & 1.468414 \\
\hline 49. & 1. & 0. & $\begin{array}{l} \\
6.605247 \\
\end{array}$ & 0.195953 & 2.310182 \\
\hline 50. & 1. & 0. & $\begin{array}{l}- \\
3.734879 \\
\end{array}$ & $\begin{array}{l}- \\
1.188177\end{array}$ & \begin{tabular}{|l|}
- \\
2.025534 \\
\end{tabular} \\
\hline 51. & 1. & 0. & 1.603239 & $\overline{0}-954290$ & 1.618093 \\
\hline 52. & 1. & 0. & \begin{tabular}{|l|}
2.526752 \\
\end{tabular} & 1.172776 & 2.073420 \\
\hline 53. & 1. & 0. & 3.261243 & 1.643273 & $\begin{array}{l}- \\
0.859550 \\
\end{array}$ \\
\hline 54. & 1. & 0. & 4.143804 & $\begin{array}{l}- \\
0.692011\end{array}$ & 0.897625 \\
\hline 55. & 1. & 0. & 2.119177 & $\begin{array}{l}- \\
0.741547\end{array}$ & $\begin{array}{l}- \\
1.388431 \\
\end{array}$ \\
\hline 56. & 1. & 0. & 1.889390 & $\begin{array}{l} \\
2.871000\end{array}$ & $\begin{array}{l} \\
0.523944 \\
\end{array}$ \\
\hline 57. & 1. & 0. & 4.421714 & 2.716564 & \begin{tabular}{|l}
0.952696 \\
\end{tabular} \\
\hline 58. & 1. & 0. & 6.085264 & 1.396394 & $\begin{array}{l} \\
0.153982\end{array}$ \\
\hline 59. & 1. & 0. & \begin{tabular}{|l|}
1.516496 \\
\end{tabular} & 3.233667 & 0.050665 \\
\hline 60. & 1. & 0. & 0.983844 & 3.076267 & 1.742293 \\
\hline 61. & 1. & 0. & 2.756706 & 4.668641 & 1.372841 \\
\hline
\end{tabular}




\begin{tabular}{|c|c|c|c|c|c|}
\hline \multicolumn{2}{|c|}{$1-6 R-5$} & \multicolumn{4}{|c|}{$\begin{array}{l}\text { Standard Orientation } \\
\text { (Ångstroms) }\end{array}$} \\
\hline $\begin{array}{l}\text { Center } \\
\text { number }\end{array}$ & $\begin{array}{c}\text { Atom } \\
\text { number }\end{array}$ & Type & $\mathrm{X}$ & Y & Z \\
\hline 1. & 6. & 0. & 2.787030 & $-\overline{1.666260}$ & 0.563624 \\
\hline 2. & 6. & 0. & 3.331828 & $\begin{array}{l}- \\
1.917741\end{array}$ & $\begin{array}{l}- \\
0.832463\end{array}$ \\
\hline 3. & 6. & 0. & 4.334016 & $\begin{array}{l}- \\
0.967600\end{array}$ & $\begin{array}{l} \\
1.338957\end{array}$ \\
\hline 4. & 6. & 0. & 4.758080 & 0.102428 & $\begin{array}{l}- \\
0.635380\end{array}$ \\
\hline 5. & 6. & 0. & 4.207378 & 0.412023 & 0.761046 \\
\hline 6. & 6. & 0. & 3.744532 & 0.904935 & 1.500994 \\
\hline 7. & 6. & 0. & 5.744459 & 1.082734 & $-\overline{1.204857}$ \\
\hline 8. & 6. & 0. & 3.070258 & 1.423839 & 0.669872 \\
\hline 9. & 6. & 0. & 2.272546 & 1.667200 & $\begin{array}{l}- \\
0.371867\end{array}$ \\
\hline 10. & 6. & 0. & 1.209094 & 2.735212 & $\begin{array}{l}- \\
0.358347\end{array}$ \\
\hline 11. & 6. & 0. & 1.628663 & 3.966881 & $\begin{array}{l}- \\
1.159288 \\
\end{array}$ \\
\hline 12. & 8. & 0. & $\begin{array}{l}-023075 \\
0.02\end{array}$ & 2.292415 & $\begin{array}{l}- \\
0.969220\end{array}$ \\
\hline 13. & 6. & 0. & 4.964329 & $\begin{array}{l}- \\
1.794076\end{array}$ & 1.836931 \\
\hline 14. & 6. & 0. & 3.022374 & $\begin{array}{l} \\
0.570191\end{array}$ & 2.818754 \\
\hline 15. & 8. & 0. & 2.912774 & $-\overline{2.839500}$ & $-\overline{1.525875}$ \\
\hline 16. & 8. & 0. & 5.195287 & 1.099855 & \begin{tabular}{|l|l|}
1.543673 \\
\end{tabular} \\
\hline 17. & 6. & 0. & $\begin{array}{l}- \\
0.699360\end{array}$ & 1.230011 & $\begin{array}{l} \\
0.376056 \\
\end{array}$ \\
\hline 18. & 8. & 0. & $\begin{array}{l}- \\
0.345436\end{array}$ & 0.028429 & $\begin{array}{l} \\
1.060091 \\
\end{array}$ \\
\hline 19. & 6. & 0. & $\begin{array}{l}- \\
1.022344\end{array}$ & 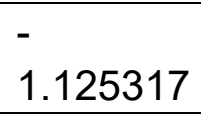 & $\begin{array}{l} \\
0.569383 \\
\end{array}$ \\
\hline 20. & 6. & 0. & $\begin{array}{l} \\
2.554349\end{array}$ & $\begin{array}{l} \\
0.958441\end{array}$ & $\begin{array}{l} \\
0.665845\end{array}$ \\
\hline
\end{tabular}




\begin{tabular}{|c|c|c|c|c|c|}
\hline 21. & 6. & 0. & $\begin{array}{l}- \\
3.016831\end{array}$ & 0.344630 & 0.013159 \\
\hline 22. & 6. & 0. & $\begin{array}{l}- \\
2.200695\end{array}$ & 1.525573 & $\begin{array}{l}- \\
0.521399\end{array}$ \\
\hline 23. & 8. & 0. & $\begin{array}{l}- \\
2.543098\end{array}$ & 2.664620 & 0.236782 \\
\hline 24. & 8. & 0. & $\begin{array}{l} \\
.208894\end{array}$ & $\begin{array}{l}- \\
2.041908\end{array}$ & 0.001543 \\
\hline 25. & 8. & 0. & $\begin{array}{l}- \\
4.399004\end{array}$ & 0.598470 & $\begin{array}{l} \\
0.357723\end{array}$ \\
\hline 26. & 16. & 0. & $\begin{array}{l}- \\
5.581465\end{array}$ & 0.092794 & 0.670968 \\
\hline 27. & 8. & 0. & $\begin{array}{l}- \\
5.050463\end{array}$ & 0.108865 & 2.026165 \\
\hline 28. & 8. & 0. & $-\overline{6.763313}$ & 0.831581 & 0.296493 \\
\hline 29. & 8. & 0. & $\begin{array}{l}- \\
5.788033\end{array}$ & $\begin{array}{l}- \\
1.417576\end{array}$ & 0.175036 \\
\hline 30. & 6. & 0. & $\begin{array}{l} \\
0.521433\end{array}$ & $\begin{array}{l}- \\
2.286845\end{array}$ & $\begin{array}{l}- \\
1.429529\end{array}$ \\
\hline 31. & 8. & 0. & $\begin{array}{l}- \\
1.197474 \\
\end{array}$ & $\begin{array}{l}- \\
3.473762 \\
\end{array}$ & $\begin{array}{l}- \\
0.990966\end{array}$ \\
\hline 32. & 1. & 0. & 2.488938 & $\begin{array}{l}- \\
2.627881\end{array}$ & 0.993531 \\
\hline 33. & 1. & 0. & 1.871071 & $\begin{array}{l}- \\
1.075980\end{array}$ & 0.429494 \\
\hline 34. & 1. & 0. & 4.703415 & $\begin{array}{l}- \\
1.161995\end{array}$ & $\begin{array}{l}- \\
2.342871\end{array}$ \\
\hline 35. & 1. & 0. & 5.999106 & 0.832891 & $\begin{array}{l}- \\
2.237062\end{array}$ \\
\hline 36. & 1. & 0. & 5.333566 & 2.097550 & $\begin{array}{l}- \\
1.172878\end{array}$ \\
\hline 37. & 1. & 0. & 6.664981 & 1.111177 & 0.612897 \\
\hline 38. & 1. & 0. & 2.944233 & 1.994179 & 1.588893 \\
\hline 39. & 1. & 0. & 2.381015 & 1.126267 & $\begin{array}{l} \\
1.309302\end{array}$ \\
\hline 40. & 1. & 0. & 0.997871 & 3.028092 & 0.680637 \\
\hline 41. & 1. & 0. & 0.821584 & 4.705136 & $\begin{array}{l}- \\
1.167505\end{array}$ \\
\hline 42. & 1. & 0. & 1.853668 & 3.691206 & 2.193666 \\
\hline 43. & 1. & 0. & 2.518543 & 4.420469 & - \\
\hline
\end{tabular}




\begin{tabular}{|c|c|c|c|c|c|}
\hline & & & & & 0.715652 \\
\hline 44. & 1. & 0. & 5.572461 & 2.029534 & 0.959072 \\
\hline 45. & 1. & 0. & 4.621087 & $\begin{array}{l}- \\
2.740951\end{array}$ & 2.264935 \\
\hline 46. & 1. & 0. & 5.606572 & $\begin{array}{l}- \\
1.328567\end{array}$ & 2.592978 \\
\hline 47. & 1. & 0. & 2.803404 & $\begin{array}{l}- \\
1.490069\end{array}$ & 3.370664 \\
\hline 48. & 1. & 0. & 3.646634 & 0.066785 & \begin{tabular}{|l|l|}
3.451687 \\
\end{tabular} \\
\hline 49. & 1. & 0. & 2.073296 & $\begin{array}{l}- \\
0.055701\end{array}$ & 2.645016 \\
\hline 50. & 1. & 0. & 5.929404 & 0.491437 & \begin{tabular}{|l|l|}
1.702185 \\
\end{tabular} \\
\hline 51. & 1. & 0. & $\begin{array}{l}- \\
0.442579\end{array}$ & 1.140017 & 0.693746 \\
\hline 52. & 1. & 0. & $\begin{array}{l}- \\
0.767214\end{array}$ & - & 0.486836 \\
\hline 53. & 1. & 0. & $\begin{array}{l}- \\
2.840569\end{array}$ & $-\overline{0.930019}$ & $\begin{array}{l} \\
1.727980 \\
\end{array}$ \\
\hline 54. & 1. & 0. & $\begin{array}{l}- \\
2.927462\end{array}$ & 0.276272 & 1.076042 \\
\hline 55. & 1. & 0. & $\begin{array}{l}- \\
2.403236\end{array}$ & 1.669074 & $\begin{array}{l} \\
1.593366\end{array}$ \\
\hline 56. & 1. & 0. & $\begin{array}{l}- \\
2.070517\end{array}$ & 3.408544 & $\begin{array}{l} \\
0.161301\end{array}$ \\
\hline 57. & 1. & 0. & $\begin{array}{l}- \\
2.791868\end{array}$ & $\begin{array}{l}- \\
2.864592\end{array}$ & $\begin{array}{l} \\
0.321930\end{array}$ \\
\hline 58. & 1. & 0. & $\begin{array}{l}- \\
4.886725\end{array}$ & $\begin{array}{l}- \\
1.846802\end{array}$ & 0.150231 \\
\hline 59. & 1. & 0. & $\begin{array}{l} \\
0.753065\end{array}$ & $\begin{array}{l} \\
2.063829\end{array}$ & $\begin{array}{l} \\
2.480626 \\
\end{array}$ \\
\hline 60. & 1. & 0. & 0.562160 & $\begin{array}{l} \\
2.399921 \\
\end{array}$ & $\begin{array}{l} \\
1.331113 \\
\end{array}$ \\
\hline 61. & 1. & 0. & $\begin{array}{l}- \\
0.877958\end{array}$ & $\begin{array}{l}- \\
4.223042 \\
\end{array}$ & \begin{tabular}{|l|}
- \\
1.507970 \\
\end{tabular} \\
\hline & & & & & \\
\hline
\end{tabular}




\section{S1. ECD Calculation Details}

\section{ECD calculation details}

\section{Methods}

Monte Carlo conformational searches were carried out by means of the Spartan's 10 software using Merck Molecular Force Field (MMFF). The conformers with Boltzmann-population of over 5\% were chosen for ECD calculations, and then the conformers were initially optimized at B3LYP/6-31g (d, p) level in $\mathrm{MeOH}$ using the CPCM polarizable conductor calculation model. The theoretical calculation of ECD was conducted in $\mathrm{MeOH}$ using Time-dependent Density functional theory (TD-DFT) at the B3LYP/6-31+g (d, p) level for all conformers of compounds 1-6S. Rotatory strengths for a total of 30 excited states were calculated. ECD spectra were generated using the program SpecDis 1.6 (University of Würzburg, Würzburg, Germany) and GraphPad Prism 5 (University of California San Diego, USA) from dipole-length rotational strengths by applying Gaussian band shapes with sigma $=0.3 \mathrm{eV}$.

\section{Results}

Table S1.2.1. Gibbs free energies ${ }^{a}$ and equilibrium populations ${ }^{b}$ of low-energy conformers of 1-6S.

\begin{tabular}{|c|l|l|}
\hline \multirow{2}{*}{ Conformers } & \multicolumn{2}{|c|}{ In $\mathrm{MeOH}$} \\
\cline { 2 - 3 } & \multicolumn{1}{|c|}{$\Delta G$} & \multicolumn{1}{|c|}{$P(\%) / 100$} \\
\hline $\mathbf{1 - 6 S}-\mathbf{1}$ & 0.00 & 0.496 \\
\hline $\mathbf{1 - 6 S}-\mathbf{2}$ & 0.79 & 0.130 \\
\hline $\mathbf{1 - 6 S}-\mathbf{3}$ & 1.23 & 0.063 \\
\hline $\mathbf{1 - 6 S}-\mathbf{4}$ & 1.36 & 0.050 \\
\hline $\mathbf{1 - 6 S}-\mathbf{5}$ & 1.36 & 0.050 \\
\hline
\end{tabular}

${ }^{a} \mathrm{~B} 3 \mathrm{LYP} / 6-31+\mathrm{G}(\mathrm{d}, \mathrm{p})$, in kcal/mol. ${ }^{b}$ From $\Delta G$ values at $298.15 \mathrm{~K}$.

Table S1.2.2. Cartesian coordinates for the low-energy reoptimized MMFF conformers of 1-6S at B3LYP/6-311+G(d,p) level of theory in $\mathrm{CH}_{3} \mathrm{OH}$.

\begin{tabular}{|c|c|c|c|c|c|}
\hline \multicolumn{2}{|c|}{ 1-6S-1 } & \multicolumn{3}{c|}{$\begin{array}{c}\text { Standard Orientation } \\
\text { (Ångstroms) }\end{array}$} \\
\hline $\begin{array}{c}\text { Center } \\
\text { number }\end{array}$ & $\begin{array}{c}\text { Atomic } \\
\text { number }\end{array}$ & $\begin{array}{c}\text { Atomic } \\
\text { Type }\end{array}$ & $\mathrm{X}$ & $\mathrm{Y}$ & $\mathrm{Z}$ \\
\hline
\end{tabular}




\begin{tabular}{|c|c|c|c|c|c|}
\hline 1. & 6. & 0. & 5.555213 & $\begin{array}{l}- \\
1.113982 \\
\end{array}$ & $\begin{array}{l}- \\
0.354090 \\
\end{array}$ \\
\hline 2. & 6. & 0. & 5.602531 & $\begin{array}{l}- \\
1.168835\end{array}$ & 1.165257 \\
\hline 3. & 6. & 0. & 4.619441 & $-\overline{0.338230}$ & 1.883380 \\
\hline 4. & 6. & 0. & 3.739441 & 0.471098 & \begin{tabular}{|l|l|}
1.261720 \\
\end{tabular} \\
\hline 5. & 6. & 0. & 3.657252 & 0.551673 & $\begin{array}{l}- \\
0.270728 \\
\end{array}$ \\
\hline 6. & 6. & 0. & 5.050999 & 0.227176 & $\begin{array}{l}- \\
0.926654\end{array}$ \\
\hline 7. & 6. & 0. & 2.767660 & 1.319378 & 2.032713 \\
\hline 8. & 6. & 0. & 2.552456 & $\begin{array}{l}- \\
0.361597\end{array}$ & $\begin{array}{l}- \\
0.790457\end{array}$ \\
\hline 9. & 6. & 0. & 1.894322 & $\begin{array}{l} \\
1.310468\end{array}$ & $\begin{array}{l} \\
0.123589 \\
\end{array}$ \\
\hline 10. & 6. & 0. & 0.743153 & $\begin{array}{l}- \\
2.085514\end{array}$ & $\begin{array}{l}- \\
0.701079\end{array}$ \\
\hline 11. & 6. & 0. & 0.945009 & $\begin{array}{l}- \\
3.594605\end{array}$ & $\begin{array}{l} \\
0.620903 \\
\end{array}$ \\
\hline 12. & 8. & 0. & $\begin{array}{l}- \\
0.476335\end{array}$ & $\begin{array}{l}- \\
1.805821\end{array}$ & 0.049616 \\
\hline 13. & 6. & 0. & 4.927434 & 0.117574 & $\begin{array}{l} \\
2.457748 \\
\end{array}$ \\
\hline 14. & 6. & 0. & 6.055632 & 1.355883 & $\begin{array}{l} \\
0.608155 \\
\end{array}$ \\
\hline 15. & 8. & 0. & 6.396462 & $\begin{array}{l}- \\
1.883234\end{array}$ & 1.762942 \\
\hline 16. & 8. & 0. & 3.322293 & 1.881718 & $\begin{array}{l} \\
0.679087 \\
\end{array}$ \\
\hline 17. & 6. & 0. & $\begin{array}{l}- \\
1.193301\end{array}$ & $\begin{array}{l}- \\
0.676035\end{array}$ & \begin{tabular}{|l|}
- \\
0.317227 \\
\end{tabular} \\
\hline 18. & 8. & 0. & $\begin{array}{l}- \\
0.599347\end{array}$ & 0.505764 & 0.198745 \\
\hline 19. & 6. & 0. & $\begin{array}{l}- \\
1.294220 \\
\end{array}$ & 1.683675 & \begin{tabular}{|l|}
- \\
0.218678 \\
\end{tabular} \\
\hline 20. & 6. & 0. & $\begin{array}{l}- \\
2.714983\end{array}$ & 1.669654 & 0.375338 \\
\hline 21. & 6. & 0. & $\begin{array}{l}- \\
3.434849\end{array}$ & 0.381843 & $\begin{array}{l}- \\
0.045117 \\
\end{array}$ \\
\hline 22. & 6. & 0. & $\begin{array}{l}- \\
2.593507\end{array}$ & $\begin{array}{l}- \\
0.843807\end{array}$ & 0.286909 \\
\hline 23. & 8. & 0. & - & - & - \\
\hline
\end{tabular}




\begin{tabular}{|c|c|c|c|c|c|}
\hline & & & 3.212994 & 2.014892 & 0.255304 \\
\hline 24. & 8. & 0. & $\begin{array}{l}- \\
3.402171\end{array}$ & 2.811475 & $\begin{array}{l}- \\
0.090715\end{array}$ \\
\hline 25. & 8. & 0. & $\begin{array}{l}- \\
4.688604\end{array}$ & 0.388954 & 0.684788 \\
\hline 26. & 16. & 0. & $-\overline{6.007754}$ & $\begin{array}{l}- \\
0.358362\end{array}$ & 0.018672 \\
\hline 27. & 8. & 0. & $\begin{array}{l} \\
7.137212\end{array}$ & 0.153255 & 0.755558 \\
\hline 28. & 8. & 0. & $\begin{array}{l} \\
5.900217\end{array}$ & $\begin{array}{l} \\
0.248204\end{array}$ & $\begin{array}{l} \\
1.427741 \\
\end{array}$ \\
\hline 29. & 8. & 0. & $\begin{array}{l}- \\
5.773814\end{array}$ & $\begin{array}{l}- \\
1.870475\end{array}$ & 0.484547 \\
\hline 30. & 6. & 0. & $\begin{array}{l}- \\
0.505928\end{array}$ & 2.897728 & 0.237226 \\
\hline 31. & 8. & 0. & 0.692426 & 2.987295 & $\begin{array}{l} \\
0.536598 \\
\end{array}$ \\
\hline 32. & 1. & 0. & 4.886211 & $\begin{array}{l} \\
1.923533 \\
\end{array}$ & $\begin{array}{l}- \\
0.678409 \\
\end{array}$ \\
\hline 33. & 1. & 0. & 6.552191 & $\begin{array}{l} \\
1.356370\end{array}$ & $\begin{array}{l} \\
0.734631\end{array}$ \\
\hline 34. & 1. & 0. & 4.658874 & $\overline{0} .396479$ & 2.968731 \\
\hline 35. & 1. & 0. & 2.933023 & 2.378049 & 1.807757 \\
\hline 36. & 1. & 0. & 1.735369 & 1.087187 & 1.744263 \\
\hline 37. & 1. & 0. & 2.873748 & 1.164881 & 3.108851 \\
\hline 38. & 1. & 0. & 2.249527 & $\begin{array}{l}- \\
0.122714\end{array}$ & $\begin{array}{l}- \\
1.809383\end{array}$ \\
\hline 39. & 1. & 0. & 2.156702 & $\begin{array}{l}- \\
1.555275\end{array}$ & 0.903820 \\
\hline 40. & 1. & 0. & 0.576169 & $\begin{array}{l} \\
1.781879 \\
\end{array}$ & $\begin{array}{l}- \\
1.744217 \\
\end{array}$ \\
\hline 41. & 1. & 0. & 1.067298 & $\begin{array}{l}- \\
3.908684 \\
\end{array}$ & 0.420009 \\
\hline 42. & 1. & 0. & 0.087459 & $\begin{array}{l}- \\
4.124269\end{array}$ & \begin{tabular}{|l|} 
\\
1.046430 \\
\end{tabular} \\
\hline 43. & 1. & 0. & 1.842616 & $\begin{array}{l}- \\
3.883703\end{array}$ & $\begin{array}{l} \\
1.174117 \\
\end{array}$ \\
\hline 44. & 1. & 0. & 4.321746 & $\begin{array}{l} \\
0.741354\end{array}$ & \begin{tabular}{|l|}
- \\
2.763093 \\
\end{tabular} \\
\hline 45. & 1. & 0. & 4.480770 & 1.024670 & $\begin{array}{l}- \\
2.873853 \\
\end{array}$ \\
\hline 46. & 1. & 0. & 5.920127 & & \\
\hline
\end{tabular}




\begin{tabular}{|c|c|c|c|c|c|}
\hline & & & & \begin{tabular}{|l|}
0.008508 \\
\end{tabular} & 2.902055 \\
\hline 47. & 1. & 0. & 5.711464 & 2.304100 & 1.025422 \\
\hline 48. & 1. & 0. & 7.028665 & 1.115693 & 1.049893 \\
\hline 49. & 1. & 0. & 6.201349 & 1.492379 & \begin{tabular}{|l}
0.466327 \\
\end{tabular} \\
\hline 50. & 1. & 0. & 2.362629 & 2.020122 & $\begin{array}{l}- \\
0.574293 \\
\end{array}$ \\
\hline 51. & 1. & 0. & $\begin{array}{l}- \\
1.264896\end{array}$ & $\begin{array}{l} \\
0.597559\end{array}$ & $\begin{array}{l}- \\
1.418307\end{array}$ \\
\hline 52. & 1. & 0. & $\begin{array}{l}- \\
1.368618\end{array}$ & 1.714208 & $\begin{array}{l}- \\
1.317022\end{array}$ \\
\hline 53. & 1. & 0. & \begin{tabular}{|l|}
- \\
2.630745 \\
\end{tabular} & 1.671777 & 1.473885 \\
\hline 54. & 1. & 0. & $\begin{array}{l}- \\
3.651988\end{array}$ & 0.417385 & $\begin{array}{l} \\
1.117762 \\
\end{array}$ \\
\hline 55. & 1. & 0. & $\begin{array}{l}- \\
2.480975\end{array}$ & $-\overline{0.934570}$ & 1.375206 \\
\hline 56. & 1. & 0. & $\begin{array}{l}- \\
2.582419\end{array}$ & $\begin{array}{l}- \\
2.740541\end{array}$ & $\begin{array}{l}- \\
0.125079\end{array}$ \\
\hline 57. & 1. & 0. & -4.308184 & 2.762110 & 0.247167 \\
\hline 58. & 1. & 0. & -4.870870 & $\begin{array}{l}- \\
2.148057\end{array}$ & 0.166700 \\
\hline 59. & 1. & 0. & $\begin{array}{l}- \\
1.135890 \\
\end{array}$ & 3.780515 & 0.086126 \\
\hline 60. & 1. & 0. & $\begin{array}{l}- \\
0.281103\end{array}$ & 2.801053 & 1.308873 \\
\hline 61. & 1. & 0. & 1.047404 & 3.880814 & $\begin{array}{l}- \\
0.445215 \\
\end{array}$ \\
\hline & & & & & \\
\hline & & & & & \\
\hline
\end{tabular}

\begin{tabular}{|l|l|l|c|l|c|}
\hline \multicolumn{2}{|c|}{$1-6 S-2$} & \multicolumn{4}{c|}{$\begin{array}{c}\text { Standard Orientation } \\
\text { (Angstroms) }\end{array}$} \\
\hline $\begin{array}{c}\text { Center } \\
\text { number }\end{array}$ & $\begin{array}{c}\text { Atom } \\
\text { number }\end{array}$ & Type & X & \multicolumn{1}{c|}{ Y } & $Z$ \\
\hline & & & & - & - \\
1. & 6. & 0. & 5.548310 & 1.053384 & 0.305353 \\
\hline & 6. & 0. & 5.539234 & - & \\
2. & 6.317752 & 1.192339 \\
\hline 3. & 6. & 0. & 4.485077 & - & 1.970176 \\
\hline
\end{tabular}




\begin{tabular}{|c|c|c|c|c|c|}
\hline & & & & 0.638592 & \\
\hline 4. & 6. & 0. & 3.609932 & 0.234914 & 1.434068 \\
\hline 5. & 6. & 0. & 3.627383 & 0.567033 & $\begin{array}{l}- \\
0.062433\end{array}$ \\
\hline 6. & 6. & 0. & 5.054720 & 0.356110 & $\begin{array}{l}- \\
0.696751\end{array}$ \\
\hline 7. & 6. & 0. & 2.537536 & 0.888750 & 2.263043 \\
\hline 8. & 6. & 0. & 2.545188 & $\begin{array}{l}- \\
0.212992\end{array}$ & $\begin{array}{l}- \\
0.793036\end{array}$ \\
\hline 9. & 6. & 0. & 1.925199 & $-\overline{1.315654}$ & $\begin{array}{l}- \\
0.377492\end{array}$ \\
\hline 10. & 6. & 0. & 0.767366 & $\begin{array}{l}- \\
1.938770\end{array}$ & $\begin{array}{l}- \\
1.110636\end{array}$ \\
\hline 11. & 6. & 0. & 0.930704 & $\begin{array}{l}- \\
3.440105\end{array}$ & $\begin{array}{l} \\
1.317417 \\
\end{array}$ \\
\hline 12. & 8. & 0. & $\begin{array}{l}- \\
0.440177\end{array}$ & $-\overline{1.758883}$ & $\begin{array}{l} \\
0.318991\end{array}$ \\
\hline 13. & 6. & 0. & 5.000370 & 0.477872 & $\begin{array}{l}- \\
2.230745 \\
\end{array}$ \\
\hline 14. & 6. & 0. & 6.043592 & 1.419768 & $\begin{array}{l} \\
0.165764\end{array}$ \\
\hline 15. & 8. & 0. & 6.338240 & $\begin{array}{l}- \\
2.075643\end{array}$ & 1.723319 \\
\hline 16. & 8. & 0. & 3.197428 & 1.932293 & $\begin{array}{l}- \\
0.265279\end{array}$ \\
\hline 17. & 6. & 0. & $\begin{array}{l}- \\
1.204014\end{array}$ & $\begin{array}{l}- \\
0.626590\end{array}$ & $\begin{array}{l}- \\
0.591968\end{array}$ \\
\hline 18. & 8. & 0. & $\begin{array}{l}- \\
0.574367\end{array}$ & 0.549639 & $\begin{array}{l} \\
0.120878 \\
\end{array}$ \\
\hline 19. & 6. & 0. & $\begin{array}{l}- \\
1.324553\end{array}$ & 1.734228 & $\begin{array}{l}- \\
0.440458\end{array}$ \\
\hline 20. & 6. & 0. & $\begin{array}{l}- \\
2.675141\end{array}$ & 1.680455 & 0.294818 \\
\hline 21. & 6. & 0. & $\begin{array}{l}- \\
3.419215\end{array}$ & 0.389279 & $\begin{array}{l}- \\
0.070676\end{array}$ \\
\hline 22. & 6. & 0. & $\begin{array}{l}- \\
2.533207\end{array}$ & $\begin{array}{l}- \\
0.830135\end{array}$ & 0.144535 \\
\hline 23. & 8. & 0. & $\begin{array}{l}- \\
3.188451\end{array}$ & $\begin{array}{l}- \\
2.000423\end{array}$ & $\begin{array}{l}- \\
0.358975\end{array}$ \\
\hline 24. & 8. & 0. & $\begin{array}{l}- \\
3.427518\end{array}$ & 2.815722 & $\begin{array}{l} \\
0.077113\end{array}$ \\
\hline 25. & 8. & 0. & $-\overline{4.587930}$ & 0.357469 & 0.792282 \\
\hline
\end{tabular}




\begin{tabular}{|c|c|c|c|c|c|}
\hline 26. & 16. & 0. & 5.959738 & $\overline{-} .395284$ & 0.259447 \\
\hline 27. & 8. & 0. & $\begin{array}{l}- \\
7.009186\end{array}$ & 0.077546 & 1.129278 \\
\hline 28. & 8. & 0. & $-\overline{6.018316}$ & $-\overline{0.253156}$ & $\begin{array}{l}- \\
1.187046\end{array}$ \\
\hline 29. & 8. & 0. & $\begin{array}{l}- \\
5.654458\end{array}$ & $\begin{array}{l}- \\
1.915075\end{array}$ & 0.659059 \\
\hline 30. & 6. & 0. & $\begin{array}{l}- \\
0.486199\end{array}$ & 2.950511 & $\begin{array}{l} \\
0.053206 \\
\end{array}$ \\
\hline 31. & 8. & 0. & 0.634777 & 3.130112 & $\overline{0}-887670$ \\
\hline 32. & 1. & 0. & 4.893340 &  & $\begin{array}{l} \\
0.766407\end{array}$ \\
\hline 33. & 1. & 0. & 6.558877 & $\begin{array}{l}- \\
1.238164\end{array}$ & $\begin{array}{l} \\
0.681626\end{array}$ \\
\hline 34. & 1. & 0. & 4.454237 & $-\overline{0.881198}$ & 3.029675 \\
\hline 35. & 1. & 0. & 2.675642 & 1.974482 & 2.310649 \\
\hline 36. & 1. & 0. & 1.550263 & 0.720510 & 1.819123 \\
\hline 37. & 1. & 0. & 2.540776 & 0.497767 & 3.282875 \\
\hline 38. & 1. & 0. & 2.224777 & 0.266524 & $\begin{array}{l} \\
1.715971\end{array}$ \\
\hline 39. & 1. & 0. & 2.196311 & $\begin{array}{l}- \\
1.791176\end{array}$ & 0.563365 \\
\hline 40. & 1. & 0. & 0.624979 & $-\overline{1.437115}$ & $\begin{array}{l}- \\
2.077316\end{array}$ \\
\hline 41. & 1. & 0. & 1.022060 & $\begin{array}{l}- \\
3.950191\end{array}$ & $\begin{array}{l}- \\
0.353510 \\
\end{array}$ \\
\hline 42. & 1. & 0. & 0.068838 & $\begin{array}{l}- \\
3.853280 \\
\end{array}$ & \begin{tabular}{|l|}
- \\
1.849233 \\
\end{tabular} \\
\hline 43. & 1. & 0. & 1.831871 & $\begin{array}{l}- \\
3.646973 \\
\end{array}$ & $\begin{array}{l}- \\
1.901690 \\
\end{array}$ \\
\hline 44. & 1. & 0. & 4.420788 & $\begin{array}{l}- \\
0.331224\end{array}$ & \begin{tabular}{|l|}
- \\
2.684303 \\
\end{tabular} \\
\hline 45. & 1. & 0. & 4.554404 & 1.430505 & \begin{tabular}{|l|}
- \\
2.531114 \\
\end{tabular} \\
\hline 46. & 1. & 0. & 6.012548 & 0.430908 & $\begin{array}{l}- \\
2.645000 \\
\end{array}$ \\
\hline 47. & 1. & 0. & 5.796855 & 2.420069 & $\begin{array}{l}- \\
0.538511\end{array}$ \\
\hline 48. & 1. & 0. & 7.052557 & 1.194612 & $\begin{array}{l} \\
0.524222 \\
\end{array}$ \\
\hline
\end{tabular}




\begin{tabular}{|c|c|c|c|c|c|}
\hline 49. & 1. & 0. & 6.083385 & 1.452607 & 0.926740 \\
\hline 50. & 1. & 0. & 3.827497 & 2.530778 & 0.157727 \\
\hline 51. & 1. & 0. & $\begin{array}{l}- \\
1.386513\end{array}$ & 0.534880 & $\begin{array}{l}- \\
1.679301\end{array}$ \\
\hline 52. & 1. & 0. & $\begin{array}{l}- \\
1.507284\end{array}$ & 1.777368 & $\begin{array}{l}- \\
1.525237\end{array}$ \\
\hline 53. & 1. & 0. & $\begin{array}{l}- \\
2.478754\end{array}$ & 1.666679 & 1.379237 \\
\hline 54. & 1. & 0. & $\begin{array}{l}- \\
3.752732\end{array}$ & 0.442933 & $\begin{array}{l}- \\
1.112002\end{array}$ \\
\hline 55. & 1. & 0. & $\begin{array}{l}- \\
2.310502\end{array}$ & $\begin{array}{l} \\
0.942719\end{array}$ & 1.213660 \\
\hline 56. & 1. & 0. & $\begin{array}{l}- \\
2.540327\end{array}$ & $\begin{array}{l} \\
\\
\end{array} .719917$ & $\begin{array}{l}- \\
0.305810\end{array}$ \\
\hline 57. & 1. & 0. & $\begin{array}{l}- \\
4.284137 \\
\end{array}$ & 2.754187 & 0.369373 \\
\hline 58. & 1. & 0. & -4.788184 & $\begin{array}{l}- \\
2.168045\end{array}$ & 0.236633 \\
\hline 59. & 1. & 0. & $\begin{array}{l}- \\
1.122950\end{array}$ & 3.833232 & $\begin{array}{l}- \\
0.159375\end{array}$ \\
\hline 60. & 1. & 0. & $-\overline{0.210462}$ & 2.862035 & 1.009807 \\
\hline 61. & 1. & 0. & 1.353616 & 2.543226 & $\begin{array}{l}- \\
0.601529\end{array}$ \\
\hline & & & & & \\
\hline
\end{tabular}

\begin{tabular}{|c|c|c|c|c|c|}
\hline \multicolumn{2}{|c|}{$1-6 S-3$} & \multicolumn{4}{|c|}{$\begin{array}{l}\text { Standard Orientation } \\
\text { (Ångstroms) }\end{array}$} \\
\hline $\begin{array}{l}\text { Center } \\
\text { number }\end{array}$ & $\begin{array}{l}\text { Atom } \\
\text { number }\end{array}$ & Type & $\mathrm{X}$ & Y & Z \\
\hline 1. & 6. & 0. & $\begin{array}{l}- \\
3.660967 \\
\end{array}$ & $\begin{array}{l}- \\
0.452652\end{array}$ & 2.168385 \\
\hline 2. & 6. & 0. & $\begin{array}{l}- \\
3.561592 \\
\end{array}$ & $\begin{array}{l}- \\
1.851311\end{array}$ & 1.570755 \\
\hline 3. & 6. & 0. & $\begin{array}{l}- \\
3.666535 \\
\end{array}$ & $\begin{array}{l}- \\
1.965108 \\
\end{array}$ & 0.104718 \\
\hline 4. & 6. & 0. & $\begin{array}{l}- \\
3.877863 \\
\end{array}$ & $\begin{array}{l}- \\
0.910921\end{array}$ & $\begin{array}{l}- \\
0.710653\end{array}$ \\
\hline 5. & 6. & 0. & $\begin{array}{l}- \\
3.923022 \\
\end{array}$ & 0.527768 & $-\overline{0.164326}$ \\
\hline 6. & 6. & 0. & - & 0.564626 & 1.318771 \\
\hline
\end{tabular}




\begin{tabular}{|c|c|c|c|c|c|}
\hline & & & 4.455188 & & \\
\hline 7. & 6. & 0. & $\begin{array}{l}- \\
4.012096\end{array}$ & $\begin{array}{l}- \\
1.076515\end{array}$ & $\begin{array}{l}- \\
2.200717\end{array}$ \\
\hline 8. & 6. & 0. & $\begin{array}{l}- \\
2.498018\end{array}$ & 1.040900 & $\begin{array}{l}- \\
0.267194\end{array}$ \\
\hline 9. & 6. & 0. & $\begin{array}{l}- \\
2.077848\end{array}$ & 1.946018 & $\begin{array}{l}- \\
1.150202\end{array}$ \\
\hline 10. & 6. & 0. & $\begin{array}{l}- \\
0.659610\end{array}$ & 2.440766 & $\begin{array}{l}- \\
1.287241\end{array}$ \\
\hline 11. & 6. & 0. & $\begin{array}{l}- \\
0.570236\end{array}$ & 3.957419 & $\begin{array}{l}-1.105403 \\
\end{array}$ \\
\hline 12. & 8. & 0. & 0.232984 & 1.846474 & $\begin{array}{l} \\
0.316671\end{array}$ \\
\hline 13. & 6. & 0. & $\begin{array}{l}- \\
4.293796\end{array}$ & 1.977688 & 1.905306 \\
\hline 14. & 6. & 0. & $\begin{array}{l}- \\
5.954815\end{array}$ & 0.190783 & 1.354268 \\
\hline 15. & 8. & 0. & $\begin{array}{l}- \\
3.378646 \\
\end{array}$ & $\begin{array}{l} \\
2.834062\end{array}$ & 2.275898 \\
\hline 16. & 8. & 0. & $\begin{array}{l} \\
4.723708\end{array}$ & 1.357768 & $\begin{array}{l} \\
1.005899 \\
\end{array}$ \\
\hline 17. & 6. & 0. & 1.037207 & 0.793429 & $\begin{array}{l} \\
0.738924 \\
\end{array}$ \\
\hline 18. & 8. & 0. & 0.331931 & $\begin{array}{l}- \\
0.431983\end{array}$ & $\begin{array}{l} \\
0.670000\end{array}$ \\
\hline 19. & 6. & 0. & 1.104290 & $\begin{array}{l} \\
1.550117\end{array}$ & $\begin{array}{l} \\
1.140082 \\
\end{array}$ \\
\hline 20. & 6. & 0. & 2.337401 & $\begin{array}{l}- \\
1.732032\end{array}$ & $\begin{array}{l}- \\
0.243075 \\
\end{array}$ \\
\hline 21. & 6. & 0. & 3.136048 & $\begin{array}{l}- \\
0.422450\end{array}$ & $\begin{array}{l} \\
0.176315 \\
\end{array}$ \\
\hline 22. & 6. & 0. & 2.234800 & 0.741863 & 0.217873 \\
\hline 23. & 8. & 0. & 2.966472 & 1.969819 & 0.136777 \\
\hline 24. & 8. & 0. & 3.115802 & $\begin{array}{l}- \\
2.777086\end{array}$ & \begin{tabular}{|l|}
- \\
0.784574 \\
\end{tabular} \\
\hline 25. & 8. & 0. & 4.175475 & $\begin{array}{l}- \\
0.653232\end{array}$ & 0.809875 \\
\hline 26. & 16. & 0. & 5.618304 & 0.140048 & \begin{tabular}{|l}
0.654572 \\
\end{tabular} \\
\hline 27. & 8. & 0. & 6.540375 & $\begin{array}{l}- \\
0.587724\end{array}$ & 1.491650 \\
\hline 28. & 8. & 0. & 5.856727 & 0.365977 & $\begin{array}{l}- \\
0.762490\end{array}$ \\
\hline 29. & 8. & 0. & 5.290293 & 1.522528 & 1.392745 \\
\hline
\end{tabular}




\begin{tabular}{|c|c|c|c|c|c|}
\hline 30. & 6. & 0. & 0.164900 & 2.752400 & $-\overline{1.126532}$ \\
\hline 31. & 8. & 0. & $\begin{array}{l}- \\
0.966662\end{array}$ & $\begin{array}{l}- \\
2.524696\end{array}$ & $\begin{array}{l}- \\
1.945001\end{array}$ \\
\hline 32. & 1. & 0. & $\begin{array}{l}- \\
2.632786\end{array}$ & $\overline{-} .097167$ & 2.318009 \\
\hline 33. & 1. & 0. & $\begin{array}{l}- \\
4.093571\end{array}$ & $-\overline{0}-547046$ & 3.169562 \\
\hline 34. & 1. & 0. & $\begin{array}{l}-574755 \\
\end{array}$ & $\begin{array}{l} \\
2.971299\end{array}$ & $\begin{array}{l}- \\
0.296035\end{array}$ \\
\hline 35. & 1. & 0. & $\begin{array}{l} \\
5.043536\end{array}$ & $\begin{array}{l}- \\
0.895898\end{array}$ & 2.525642 \\
\hline 36. & 1. & 0. & $\begin{array}{l}- \\
3.392078\end{array}$ & $\begin{array}{l}- \\
0.348654\end{array}$ & 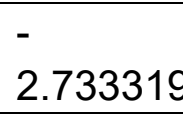 \\
\hline 37. & 1. & 0. & $\begin{array}{l}- \\
3.726681\end{array}$ & $\begin{array}{l}- \\
2.083838 \\
\end{array}$ & $\begin{array}{l}- \\
2.510534\end{array}$ \\
\hline 38. & 1. & 0. & - 1.778531 & 0.576035 & 0.397312 \\
\hline 39. & 1. & 0. & $\begin{array}{l}- \\
2.791900\end{array}$ & 2.403650 & $\begin{array}{l}- \\
1.830631\end{array}$ \\
\hline 40. & 1. & 0. & $\begin{array}{l}- \\
0.289790\end{array}$ & 2.183099 & $\begin{array}{l}- \\
2.292181\end{array}$ \\
\hline 41. & 1. & 0. & $\begin{array}{l}- \\
0.862293\end{array}$ & 4.230580 & $\begin{array}{l}- \\
0.087489\end{array}$ \\
\hline 42. & 1. & 0. & 0.449078 & 4.309388 & $\begin{array}{l} \\
1.290833 \\
\end{array}$ \\
\hline 43. & 1. & 0. & $\begin{array}{l}- \\
1.241647\end{array}$ & 4.467349 & $\begin{array}{l}- \\
1.801994 \\
\end{array}$ \\
\hline 44. & 1. & 0. & $\begin{array}{l}- \\
3.241871\end{array}$ & 2.267626 & 1.980192 \\
\hline 45. & 1. & 0. & $\begin{array}{l}- \\
4.801807\end{array}$ & 2.714513 & 1.277033 \\
\hline 46. & 1. & 0. & -4.727943 & 2.023003 & 2.909566 \\
\hline 47. & 1. & 0. & $\begin{array}{l}- \\
6.570055\end{array}$ & 0.944124 & 0.849915 \\
\hline 48. & 1. & 0. & $\begin{array}{l}- \\
6.300441\end{array}$ & 0.149797 & 2.391618 \\
\hline 49. & 1. & 0. & $\begin{array}{l}- \\
6.157050\end{array}$ & $\begin{array}{l}- \\
0.785395\end{array}$ & 0.903203 \\
\hline 50. & 1. & 0. & $\begin{array}{l}- \\
5.623417\end{array}$ & 1.007611 & $\begin{array}{l}- \\
1.003382\end{array}$ \\
\hline 51. & 1. & 0. & 1.390676 & 0.961848 & $\begin{array}{l}- \\
1.772594\end{array}$ \\
\hline
\end{tabular}




\begin{tabular}{|c|c|c|c|c|c|}
\hline 52. & 1. & 0. & 1.432358 & $\begin{array}{l} \\
1.362751\end{array}$ & $\begin{array}{l} \\
2.174032 \\
\end{array}$ \\
\hline 53. & 1. & 0. & 1.993001 & $\begin{array}{l}- \\
1.972791\end{array}$ & 0.775462 \\
\hline 54. & 1. & 0. & 3.607317 & $-\overline{0.229939}$ & $\begin{array}{l}- \\
1.145545 \\
\end{array}$ \\
\hline 55. & 1. & 0. & 1.851097 & 0.587454 & 1.234920 \\
\hline 56. & 1. & 0. & 2.326735 & 2.680143 & \begin{tabular}{|l|l|}
0.299640 \\
\end{tabular} \\
\hline 57. & 1. & 0. & 3.895323 & $\begin{array}{l}- \\
2.880895\end{array}$ & $\begin{array}{l}- \\
0.220298\end{array}$ \\
\hline 58. & 1. & 0. & 4.488341 & 1.914166 & 0.951991 \\
\hline 59. & 1. & 0. & 0.693707 & $\begin{array}{l}- \\
3.621729\end{array}$ & $\begin{array}{l}-522638 \\
1.52\end{array}$ \\
\hline 60. & 1. & 0. & $\begin{array}{l}- \\
0.122620\end{array}$ & $\begin{array}{l}- \\
2.969567\end{array}$ & $\begin{array}{l}- \\
0.085736\end{array}$ \\
\hline 61. & 1. & 0. & $\begin{array}{l}- \\
1.394442\end{array}$ & $\begin{array}{l}- \\
1.730962\end{array}$ & $\begin{array}{l} \\
1.593652\end{array}$ \\
\hline & & & & & \\
\hline
\end{tabular}

\begin{tabular}{|l|l|l|c|l|l|}
\hline \multicolumn{2}{|c|}{$1-6 S-4$} & \multicolumn{4}{c|}{$\begin{array}{c}\text { Standard Orientation } \\
\text { (Angstroms) }\end{array}$} \\
\hline $\begin{array}{c}\text { Center } \\
\text { number }\end{array}$ & $\begin{array}{c}\text { Atom } \\
\text { number }\end{array}$ & \multicolumn{1}{|c|}{ Type } & X & \multicolumn{1}{c|}{ Y } & \multicolumn{1}{c|}{ Z } \\
\hline 1. & 6. & 0. & 2.880294 & 1.444559 & 0.859646 \\
\hline 2. & 6. & 0. & 3.742136 & 1.826118 & 0.328504 \\
\hline 3. & 6. & 0. & 4.745310 & 0.854464 & 0.782547 \\
\hline 4. & 6. & 0. & 4.811772 & 0.399575 & 0.290424 \\
\hline 5. & 6. & 0. & 3.817500 & 0.885260 & 0.771702 \\
\hline 6. & 6. & 0. & 3.425294 & 0.297481 & 1.734324 \\
\hline 7. & 6. & 0. & 5.823328 & 1.392465 & 0.785639 \\
\hline 8. & 6. & 0. & 2.579220 & 1.492479 & 0.126186 \\
\hline 9. & 6. & 0. & 2.198472 & 1.405084 & 1.149511 \\
\hline 10. & 6. & 0. & 0.901243 & - & - \\
\hline
\end{tabular}




\begin{tabular}{|c|c|c|c|c|c|}
\hline 11. & 6. & 0. & 0.998919 & $\begin{array}{l}- \\
3.479237 \\
\end{array}$ & \begin{tabular}{|l|}
- \\
1.970455 \\
\end{tabular} \\
\hline 12. & 8. & 0. & $\begin{array}{l}- \\
0.180337\end{array}$ & $\begin{array}{l}- \\
1.805325\end{array}$ & $\begin{array}{l}- \\
0.728495 \\
\end{array}$ \\
\hline 13. & 6. & 0. & 2.334630 & $\begin{array}{l}- \\
0.134777\end{array}$ & 2.733487 \\
\hline 14. & 6. & 0. & 4.659984 & 0.755185 & \begin{tabular}{|l|}
2.540674 \\
\end{tabular} \\
\hline 15. & 8. & 0. & 3.576381 & 2.894032 & $\begin{array}{l}- \\
0.917673\end{array}$ \\
\hline 16. & 8. & 0. & 4.426298 & $\begin{array}{l}- \\
1.886212\end{array}$ & 1.605698 \\
\hline 17. & 6. & 0. & $\begin{array}{l}- \\
0.977152\end{array}$ & $\begin{array}{l}- \\
0.680488\end{array}$ & $\begin{array}{l} \\
0.936385 \\
\end{array}$ \\
\hline 18. & 8. & 0. & $\begin{array}{l}-276906 \\
0.27\end{array}$ & 0.506751 & $\begin{array}{l}- \\
0.634975\end{array}$ \\
\hline 19. & 6. & 0. & $\begin{array}{l}- \\
1.047723\end{array}$ & 1.678665 & $\begin{array}{l} \\
0.951033\end{array}$ \\
\hline 20. & 6. & 0. & $\begin{array}{l} \\
2.280148\end{array}$ & 1.711554 & $\begin{array}{l}- \\
0.031411 \\
\end{array}$ \\
\hline 21. & 6. & 0. & $\begin{array}{l}- \\
3.078236\end{array}$ & 0.405260 & $\begin{array}{l} \\
0.174261\end{array}$ \\
\hline 22. & 6. & 0. & $\begin{array}{l}- \\
2.182572\end{array}$ & $\begin{array}{l}- \\
0.815984\end{array}$ & $\begin{array}{l} \\
0.001024\end{array}$ \\
\hline 23. & 8. & 0. & $\begin{array}{l}- \\
2.917529\end{array}$ & $\begin{array}{l}- \\
2.003223\end{array}$ & \begin{tabular}{|l} 
\\
0.324171 \\
\end{tabular} \\
\hline 24. & 8. & 0. & $\begin{array}{l}- \\
3.075073\end{array}$ & 2.822591 & $\begin{array}{l}- \\
0.385914\end{array}$ \\
\hline 25. & 8. & 0. & $\begin{array}{l}- \\
4.103538\end{array}$ & 0.464291 & 0.853190 \\
\hline 26. & 16. & 0. & $\begin{array}{l}- \\
5.546184\end{array}$ & $\begin{array}{l} \\
0.298894\end{array}$ & 0.592262 \\
\hline 27. & 8. & 0. & $\begin{array}{l} \\
6.445660\end{array}$ & 0.253492 & 1.575675 \\
\hline 28. & 8. & 0. & $\begin{array}{l}- \\
5.825430\end{array}$ & $\begin{array}{l}- \\
0.259069\end{array}$ & $\begin{array}{l} \\
0.834712 \\
\end{array}$ \\
\hline 29. & 8. & 0. & $\begin{array}{l}- \\
5.195068\end{array}$ & $\begin{array}{l}- \\
1.792397\end{array}$ & 1.050616 \\
\hline 30. & 6. & 0. & $\begin{array}{l}- \\
0.152867\end{array}$ & 2.902622 & $\begin{array}{l}- \\
0.797988\end{array}$ \\
\hline 31. & 8. & 0. & 0.853519 & 2.856535 & $\begin{array}{l} \\
1.786093\end{array}$ \\
\hline 32. & 1. & 0. & 1.905550 & 1.158890 & 0.444232 \\
\hline 33. & 1. & 0. & 2.718715 & 2.348388 & 1.457696 \\
\hline
\end{tabular}




\begin{tabular}{|c|c|c|c|c|c|}
\hline 34. & 1. & 0. & 5.413730 & 1.190666 & $\begin{array}{l}- \\
1.571183 \\
\end{array}$ \\
\hline 35. & 1. & 0. & 6.465930 & $\begin{array}{l}- \\
1.720075\end{array}$ & 0.038138 \\
\hline 36. & 1. & 0. & 5.330792 & $\begin{array}{l}- \\
2.289814\end{array}$ & $\begin{array}{l}- \\
1.182304 \\
\end{array}$ \\
\hline 37. & 1. & 0. & 6.446454 & $\begin{array}{l}- \\
0.967826\end{array}$ & $\begin{array}{l}- \\
1.575325\end{array}$ \\
\hline 38. & 1. & 0. & 1.933953 & $\begin{array}{l}- \\
2.010857\end{array}$ & 0.832234 \\
\hline 39. & 1. & 0. & 2.814639 & $\begin{array}{l} \\
0.883144\end{array}$ & $\begin{array}{l} \\
1.877679\end{array}$ \\
\hline 40. & 1. & 0. & 0.633270 & $\begin{array}{l}- \\
1.450071\end{array}$ & $\begin{array}{l}- \\
2.598707\end{array}$ \\
\hline 41. & 1. & 0. & 1.200774 & $\begin{array}{l}- \\
4.030494\end{array}$ & $\begin{array}{l}- \\
1.047145\end{array}$ \\
\hline 42. & 1. & 0. & 0.067698 & $\begin{array}{l}- \\
3.850422\end{array}$ & $\begin{array}{l}- \\
2.408675 \\
\end{array}$ \\
\hline 43. & 1. & 0. & 1.814980 & $\begin{array}{l}- \\
3.678464\end{array}$ & $\begin{array}{l}- \\
2.671229 \\
\end{array}$ \\
\hline 44. & 1. & 0. & 1.382650 & $\begin{array}{l}- \\
0.344228\end{array}$ & 2.237539 \\
\hline 45. & 1. & 0. & 2.646706 & $\begin{array}{l} \\
1.023520\end{array}$ & 3.288133 \\
\hline 46. & 1. & 0. & 2.156783 & 0.669564 & 3.454886 \\
\hline 47. & 1. & 0. & 5.041854 & $\overline{-}-061673$ & 3.156800 \\
\hline 48. & 1. & 0. & 4.378143 & 1.580410 & 3.203001 \\
\hline 49. & 1. & 0. & 5.472607 & 1.105140 & 1.899330 \\
\hline 50. & 1. & 0. & 4.392228 & $\begin{array}{l} \\
2.728640\end{array}$ & 1.133673 \\
\hline 51. & 1. & 0. & $\begin{array}{l} \\
1.322153 \\
\end{array}$ & $\begin{array}{l}- \\
0.645897\end{array}$ & $\begin{array}{l}- \\
1.987170 \\
\end{array}$ \\
\hline 52. & 1. & 0. & $\begin{array}{l}- \\
1.380340\end{array}$ & 1.628273 & $\begin{array}{l}- \\
1.999342 \\
\end{array}$ \\
\hline 53. & 1. & 0. & $\begin{array}{l}- \\
1.929640\end{array}$ & 1.784090 & 1.010845 \\
\hline 54. & 1. & 0. & $\begin{array}{l}- \\
3.564081 \\
\end{array}$ & 0.383785 & \begin{tabular}{|l|}
- \\
1.155109 \\
\end{tabular} \\
\hline 55. & 1. & 0. & $\begin{array}{l}- \\
1.809248\end{array}$ & $\begin{array}{l} \\
0.861496\end{array}$ & 1.030498 \\
\hline 56. & 1. & 0. & 2.276533 & $\begin{array}{l}- \\
2.730690\end{array}$ & 0.329797 \\
\hline
\end{tabular}




\begin{tabular}{|c|c|c|c|c|c|}
\hline 57. & 1. & 0. & 3.854831 & 2.816186 & 0.187540 \\
\hline 58. & 1. & 0. & $\overline{4} .409149$ & $\begin{array}{l}- \\
2.094120\end{array}$ & 0.517678 \\
\hline 59. & 1. & 0. & $\begin{array}{l}- \\
0.787794\end{array}$ & 3.792919 & $\begin{array}{l}- \\
0.904685\end{array}$ \\
\hline 60. & 1. & 0 . & 0.259522 & 2.908726 & 0.221177 \\
\hline 61. & 1. & 0. & 1.706058 & 3.127918 & $\begin{array}{l}- \\
1.405603 \\
\end{array}$ \\
\hline & & & & & \\
\hline
\end{tabular}

\begin{tabular}{|c|c|c|c|c|c|}
\hline \multicolumn{2}{|c|}{$1-6 S-5$} & \multicolumn{4}{|c|}{$\begin{array}{l}\text { Standard Orientation } \\
\text { (Ångstroms) }\end{array}$} \\
\hline $\begin{array}{l}\text { Center } \\
\text { number }\end{array}$ & $\begin{array}{l}\text { Atom } \\
\text { number }\end{array}$ & Type & $\mathrm{X}$ & $\mathrm{Y}$ & Z \\
\hline 1. & 6. & 0. & 3.226890 & $\begin{array}{l}- \\
1.528392 \\
\end{array}$ & 0.998021 \\
\hline 2. & 6. & 0. & $\begin{array}{l}- \\
3.992101\end{array}$ & $\begin{array}{l}- \\
2.072180\end{array}$ & $\begin{array}{l}- \\
0.198881\end{array}$ \\
\hline 3. & 6. & 0. & $-\overline{4.946918}$ & $-\overline{1}-180075$ & $\begin{array}{l}- \\
0.868344\end{array}$ \\
\hline 4. & 6. & 0. & $\begin{array}{l}- \\
4.991459\end{array}$ & 0.143719 & $\begin{array}{l}- \\
0.616198\end{array}$ \\
\hline 5. & 6. & 0. & - & 0.783786 & 0.373877 \\
\hline 6. & 6. & 0. & $\begin{array}{l}- \\
3.735407 \\
\end{array}$ & $\begin{array}{l}- \\
0.194039\end{array}$ & 1.580582 \\
\hline 7. & 6. & 0. & $\begin{array}{l}- \\
5.933205\end{array}$ & 1.064442 & $\begin{array}{l}- \\
1.334228 \\
\end{array}$ \\
\hline 8. & 6. & 0. & $\begin{array}{l}- \\
2.721050\end{array}$ & 1.067297 & $\begin{array}{l}- \\
0.404950\end{array}$ \\
\hline 9. & 6. & 0. & $\begin{array}{l}- \\
1.994567\end{array}$ & 2.182462 & $\begin{array}{l}- \\
0.309270\end{array}$ \\
\hline 10. & 6. & 0. & $\begin{array}{l}- \\
0.707074\end{array}$ & 2.387679 & $\begin{array}{l}- \\
1.067789 \\
\end{array}$ \\
\hline 11. & 6. & 0. & $\begin{array}{l}- \\
0.489499\end{array}$ & 3.835881 & $\begin{array}{l} \\
1.489325 \\
\end{array}$ \\
\hline 12. & 8. & 0. & 0.419322 & 2.008486 & $\begin{array}{l}- \\
0.216727\end{array}$ \\
\hline 13. & 6. & 0. & $-\overline{2.670167}$ & 0.383115 & 2.529534 \\
\hline
\end{tabular}




\begin{tabular}{|c|c|c|c|c|c|}
\hline 14. & 6. & 0. & $\begin{array}{l}- \\
5.038548\end{array}$ & $\begin{array}{l}- \\
0.407730\end{array}$ & 2.380306 \\
\hline 15. & 8. & 0. & $\begin{array}{l}- \\
3.775694 \\
\end{array}$ & $\begin{array}{l}- \\
3.209758\end{array}$ & $\begin{array}{l}- \\
0.616363 \\
\end{array}$ \\
\hline 16. & 8. & 0. & $-\overline{4.538488}$ & 1.977542 & 0.939990 \\
\hline 17. & 6. & 0. & 1.102240 & 0.862537 & $\begin{array}{l}- \\
0.607840\end{array}$ \\
\hline 18. & 8. & 0. & 0.335209 & $\begin{array}{l}- \\
0.297086\end{array}$ & $\begin{array}{l}- \\
0.347993\end{array}$ \\
\hline 19. & 6. & 0. & 0.973459 & $\begin{array}{l}- \\
1.487666\end{array}$ & $\begin{array}{l}- \\
0.832655\end{array}$ \\
\hline 20. & 6. & 0. & 2.288144 & $\begin{array}{l}- \\
1.694626\end{array}$ &  \\
\hline 21. & 6. & 0. & 3.168640 & $\begin{array}{l}- \\
0.441262\end{array}$ & $\begin{array}{l}- \\
0.173491\end{array}$ \\
\hline 22. & 6. & 0. & 2.394935 & 0.813123 & 0.212453 \\
\hline 23. & 8. & 0. & 3.188244 & 1.974142 & $\begin{array}{l}- \\
0.062697\end{array}$ \\
\hline 24. & 8. & 0. & 2.947922 & $\begin{array}{l}- \\
2.821972\end{array}$ & $\begin{array}{l}- \\
0.595424 \\
\end{array}$ \\
\hline 25. & 8. & 0. & 4.288051 & $-\overline{0.678310}$ & 0.721435 \\
\hline 26. & 16. & 0. & 5.751427 & 0.008286 & 0.374695 \\
\hline 27. & 8. & 0. & 6.703969 & $\overline{0}-709653$ & 1.186302 \\
\hline 28. & 8. & 0. & 5.871657 & 0.105886 & $\begin{array}{l} \\
1.071665\end{array}$ \\
\hline 29. & 8. & 0. & 5.572514 & 1.462155 & 1.019544 \\
\hline 30. & 6. & 0. & 0.008948 & $\begin{array}{l}- \\
2.649733\end{array}$ & $\begin{array}{l}- \\
0.658733\end{array}$ \\
\hline 31. & 8. & 0. & $\begin{array}{l}- \\
1.115756\end{array}$ & $\begin{array}{l}- \\
2.414753\end{array}$ & $\begin{array}{l}- \\
1.485022 \\
\end{array}$ \\
\hline 32. & 1. & 0. & $\begin{array}{l}- \\
2.190652\end{array}$ & $\begin{array}{l} \\
1.414176\end{array}$ & 0.659158 \\
\hline 33. & 1. & 0. & $\begin{array}{l}- \\
3.217890\end{array}$ & $\begin{array}{l}- \\
2.309698\end{array}$ & 1.766445 \\
\hline 34. & 1. & 0. & $\begin{array}{l}- \\
5.579577\end{array}$ & $\begin{array}{l}- \\
1.635747\end{array}$ & $\begin{array}{l} \\
1.625758 \\
\end{array}$ \\
\hline 35. & 1. & 0. & $\begin{array}{l}- \\
6.583567\end{array}$ & 1.577572 & $\begin{array}{l} \\
0.617938\end{array}$ \\
\hline 36. & 1. & 0. & $\begin{array}{l}- \\
5.382037\end{array}$ & 1.841881 & $\begin{array}{l} \\
1.880511\end{array}$ \\
\hline
\end{tabular}




\begin{tabular}{|c|c|c|c|c|c|}
\hline 37. & 1. & 0. & $\begin{array}{l}- \\
6.552917\end{array}$ & 0.522452 & $\begin{array}{l}- \\
2.051350\end{array}$ \\
\hline 38. & 1. & 0. & $\begin{array}{l}- \\
2.377342\end{array}$ & 0.267207 & $\begin{array}{l}- \\
1.057272\end{array}$ \\
\hline 39. & 1. & 0. & $\begin{array}{l}- \\
2.285243\end{array}$ & 2.973584 & 0.380422 \\
\hline 40. & 1. & 0. & $\begin{array}{l}- \\
0.699551\end{array}$ & 1.733258 & $\begin{array}{l}- \\
1.946696\end{array}$ \\
\hline 41. & 1. & 0. & $\begin{array}{l} \\
0.437157\end{array}$ & 4.484590 & $\begin{array}{l}- \\
0.608914\end{array}$ \\
\hline 42. & 1. & 0. & 0.441576 & 3.941681 & $\begin{array}{l}- \\
2.053547\end{array}$ \\
\hline 43. & 1. & 0. & $\begin{array}{l}- \\
1.315457\end{array}$ & 4.180032 & $\begin{array}{l}- \\
2.119582\end{array}$ \\
\hline 44. & 1. & 0. & $\begin{array}{l}- \\
1.709724\end{array}$ & 0.522544 & 2.027261 \\
\hline 45. & 1. & 0. & $\begin{array}{l}- \\
2.993172\end{array}$ & 1.345206 & 2.935206 \\
\hline 46. & 1. & 0. & $\begin{array}{l}- \\
2.517137\end{array}$ & $\begin{array}{l}- \\
0.304384\end{array}$ & 3.368337 \\
\hline 47. & 1. & 0. & 5.409805 & 0.541021 & 2.773659 \\
\hline 48. & 1. & 0. & $\begin{array}{l}- \\
4.842120\end{array}$ & $\begin{array}{l} \\
1.075153\end{array}$ & 3.226039 \\
\hline 49. & 1. & 0. & $\begin{array}{l}- \\
5.829908\end{array}$ & $\begin{array}{l} \\
0.859422\end{array}$ & 1.776710 \\
\hline 50. & 1. & 0. & $\begin{array}{l}- \\
4.502785\end{array}$ & 2.668700 & 0.265051 \\
\hline 51. & 1. & 0. & 1.348785 & 0.904056 & \begin{tabular}{|l|}
- \\
1.685968 \\
\end{tabular} \\
\hline 52. & 1. & 0. & 1.198932 & $\begin{array}{l}-378982 \\
\end{array}$ & $\begin{array}{l} \\
.905042 \\
\end{array}$ \\
\hline 53. & 1. & 0. & 2.041766 & $\begin{array}{l}- \\
1.841185\end{array}$ & 1.003736 \\
\hline 54. & 1. & 0. & 3.549375 & $\begin{array}{l}- \\
0.354028\end{array}$ & $\begin{array}{l}1.196248 \\
\end{array}$ \\
\hline 55. & 1. & 0. & 2.122253 & 0.774122 & 1.275066 \\
\hline 56. & 1. & 0. & 2.607168 & 2.739310 & \begin{tabular}{|l}
0.069203 \\
\end{tabular} \\
\hline 57. & 1. & 0. & 3.784018 & $\begin{array}{l}- \\
2.921436\end{array}$ & $\begin{array}{l}- \\
0.117811\end{array}$ \\
\hline 58. & 1. & 0. & 4.759438 & 1.873251 & 0.615556 \\
\hline 59. & 1. & 0. & 0.539943 & $\begin{array}{l}- \\
3.571915\end{array}$ & $\begin{array}{l} \\
0.930285\end{array}$ \\
\hline
\end{tabular}




\begin{tabular}{|c|c|c|c|c|c|}
\hline 60. & 1. & 0. & $\begin{array}{l}- \\
0.267310\end{array}$ & $\begin{array}{l} \\
2.717818\end{array}$ & 0.405063 \\
\hline 61. & 1. & 0. & $\begin{array}{l}- \\
1.831042 \\
\end{array}$ & $\begin{array}{l}- \\
3.022545\end{array}$ & $\begin{array}{l}- \\
1.233793 \\
\end{array}$ \\
\hline & & & & & \\
\hline
\end{tabular}

S1. ECD Calculation Details

\section{ECD calculation details}

\section{Methods}

Monte Carlo conformational searches were carried out by means of the Spartan's 10 software using Merck Molecular Force Field (MMFF). The conformers with Boltzmann-population of over 5\% were chosen for ECD calculations, and then the conformers were initially optimized at B3LYP/6-31g (d, p) level in $\mathrm{MeOH}$ using the CPCM polarizable conductor calculation model. The theoretical calculation of ECD was conducted in $\mathrm{MeOH}$ using Time-dependent Density functional theory (TD-DFT) at the B3LYP/6-31+g $(\mathrm{d}, \mathrm{p})$ level for all conformers of compounds 3-6R. Rotatory strengths for a total of 30 excited states were calculated. ECD spectra were generated using the program SpecDis 1.6 (University of Würzburg, Würzburg, Germany) and GraphPad Prism 5 (University of California San Diego, USA) from dipole-length rotational strengths by applying Gaussian band shapes with sigma $=0.3 \mathrm{eV}$.

\section{Results}

Table S1.2.1. Gibbs free energies ${ }^{a}$ and equilibrium populations ${ }^{b}$ of low-energy conformers of 3-6R.

\begin{tabular}{|c|c|c|}
\hline \multirow{2}{*}{ Conformers } & \multicolumn{2}{|c|}{ In $\mathrm{MeOH}$} \\
\cline { 2 - 3 } & $\Delta G$ & $P(\%) / 100$ \\
\hline 3-6R-1 & 0.00 & 0.295 \\
\hline 3-6R-2 & 0.29 & 0.181 \\
\hline $\mathbf{3 - 6 R - 3}$ & 0.30 & 0.178 \\
\hline $\mathbf{3 - 6} \boldsymbol{R}-\mathbf{4}$ & 0.74 & 0.085 \\
\hline $\mathbf{3 - 6} \boldsymbol{R}-\mathbf{5}$ & 0.85 & 0.071 \\
\hline
\end{tabular}

${ }^{a} \mathrm{~B} 3 \mathrm{LYP} / 6-31+\mathrm{G}(\mathrm{d}, \mathrm{p})$, in $\mathrm{kcal} / \mathrm{mol} .{ }^{b}$ From $\Delta G$ values at $298.15 \mathrm{~K}$. 
Table S1.2.2. Cartesian coordinates for the low-energy reoptimized MMFF conformers of 3-6R at B3LYP/6-311+G(d,p) level of theory in $\mathrm{CH}_{3} \mathrm{OH}$.

\begin{tabular}{|c|c|c|c|c|c|}
\hline \multicolumn{2}{|c|}{$3-6 R-1$} & \multicolumn{4}{|c|}{$\begin{array}{c}\text { Standard Orientation } \\
\text { (Ångstroms) }\end{array}$} \\
\hline $\begin{array}{l}\text { Center } \\
\text { number }\end{array}$ & $\begin{array}{l}\text { Atomic } \\
\text { number }\end{array}$ & $\begin{array}{l}\text { Atomic } \\
\text { Type }\end{array}$ & $X$ & Y & Z \\
\hline 1. & 6. & 0. & - & $\begin{array}{l}- \\
1.688909\end{array}$ & 0.250012 \\
\hline 2. & 6. & 0. & $\begin{array}{l}- \\
5.626346\end{array}$ & $\begin{array}{l}- \\
1.347766\end{array}$ & $\begin{array}{l}- \\
0.609552\end{array}$ \\
\hline 3. & 6. & 0. & $\begin{array}{l}- \\
5.980695\end{array}$ & 0.078676 & $\begin{array}{l}- \\
0.743942\end{array}$ \\
\hline 4. & 6. & 0. & $\begin{array}{l}- \\
5.313785\end{array}$ & 1.071468 & $\begin{array}{l}- \\
0.123982 \\
\end{array}$ \\
\hline 5. & 6. & 0. & $\begin{array}{l}- \\
4.069515\end{array}$ & 0.787856 & 0.712684 \\
\hline 6. & 6. & 0. & $\begin{array}{l}- \\
4.093782\end{array}$ & $\begin{array}{l}- \\
0.646955 \\
\end{array}$ & 1.344531 \\
\hline 7. & 6. & 0. & $\begin{array}{l}- \\
5.719464\end{array}$ & 2.512541 & $\begin{array}{l}- \\
0.253733\end{array}$ \\
\hline 8. & 6. & 0. & $\begin{array}{l}- \\
2.858596\end{array}$ & 1.068101 & $\begin{array}{l}- \\
0.156097\end{array}$ \\
\hline 9. & 6. & 0. & $\begin{array}{l}- \\
1.913947\end{array}$ & 1.971271 & 0.117999 \\
\hline 10. & 6. & 0. & $\begin{array}{l}- \\
0.731632\end{array}$ & 2.272570 & $\begin{array}{l}- \\
0.762176\end{array}$ \\
\hline 11. & 6. & 0. & $\begin{array}{l}- \\
0.628657\end{array}$ & 3.750460 & $\begin{array}{l}- \\
1.128178\end{array}$ \\
\hline 12. & 8. & 0. & 0.489068 & 1.922026 & $\begin{array}{l}- \\
0.040559\end{array}$ \\
\hline 13. & 6. & 0. & $\begin{array}{l}- \\
2.742654\end{array}$ & $\begin{array}{l}- \\
0.973281\end{array}$ & 2.003228 \\
\hline 14. & 6. & 0. & $\begin{array}{l}- \\
5.184008 \\
\end{array}$ & $\begin{array}{l}- \\
0.689087 \\
\end{array}$ & 2.437313 \\
\hline 15. & 8. & 0. & $\begin{array}{l}- \\
6.272508\end{array}$ & $\begin{array}{l}- \\
2.217327 \\
\end{array}$ & $\begin{array}{l}- \\
1.177421\end{array}$ \\
\hline 16. & 1. & 0. & $\begin{array}{l}- \\
4.043498\end{array}$ & 1.507991 & 1.542288 \\
\hline 17. & 6. & 0. & 1.188805 & 0.828818 & $\begin{array}{l}- \\
0.529228\end{array}$ \\
\hline 18. & 8. & 0. & 0.456260 & $\begin{array}{l}- \\
0.367167 \\
\end{array}$ & $\begin{array}{l}- \\
0.321999\end{array}$ \\
\hline 19. & 6. & 0. & 1.122807 & - & - \\
\hline
\end{tabular}




\begin{tabular}{|c|c|c|c|c|c|}
\hline & & & & 1.523663 & 0.858093 \\
\hline 20. & 6. & 0. & 2.438717 & $\begin{array}{l}- \\
1.736617\end{array}$ & $\begin{array}{l}- \\
0.100715\end{array}$ \\
\hline 21. & 6. & 0. & 3.295568 & $-\overline{0.464607}$ & $\begin{array}{l}- \\
0.190819\end{array}$ \\
\hline 22. & 6. & 0. & 2.504793 & 0.761386 & \begin{tabular}{|l|l|}
0.253878 \\
\end{tabular} \\
\hline 23. & 8. & 0. & 3.271036 & 1.945949 & 0.006601 \\
\hline 24. & 8. & 0. & 3.099508 & $\begin{array}{l}- \\
2.840144 \\
\end{array}$ & $\begin{array}{l}- \\
0.679530\end{array}$ \\
\hline 25. & 8. & 0. & 4.434726 & $\begin{array}{l}- \\
0.705348\end{array}$ & 0.674897 \\
\hline 26. & 16. & 0. & 5.881411 & 0.013121 & \begin{tabular}{|l}
0.315412 \\
\end{tabular} \\
\hline 27. & 8. & 0. & 6.862776 & 0.717514 & 1.079271 \\
\hline 28. & 8. & 0. & 5.962194 & 0.160477 & $\begin{array}{l}- \\
1.129337 \\
\end{array}$ \\
\hline 29. & 8. & 0. & 5.697050 & 1.441822 & \begin{tabular}{|l|l|}
1.013117 \\
\end{tabular} \\
\hline 30. & 6. & 0. & 0.126153 & $\begin{array}{l}- \\
2.668871\end{array}$ & $\begin{array}{l}- \\
0.723901\end{array}$ \\
\hline 31. & 8. & 0. & $\begin{array}{l}- \\
1.077049\end{array}$ & $\begin{array}{l}- \\
2.355301\end{array}$ & $\begin{array}{l} \\
1.405125 \\
\end{array}$ \\
\hline 32. & 1. & 0. & $\begin{array}{l}- \\
3.556490\end{array}$ & $\begin{array}{l}- \\
1.817767\end{array}$ & $\begin{array}{l}- \\
0.425071\end{array}$ \\
\hline 33. & 1. & 0. & $\begin{array}{l} \\
4.588921\end{array}$ & $\begin{array}{l}- \\
2.676501\end{array}$ & 0.689191 \\
\hline 34. & 1. & 0. & $\begin{array}{l}- \\
6.848060\end{array}$ & 0.286411 & $\begin{array}{l} \\
1.365818\end{array}$ \\
\hline 35. & 1. & 0. & $\begin{array}{l}- \\
6.028995\end{array}$ & 2.912263 & 0.720839 \\
\hline 36. & 1. & 0. & $\begin{array}{l}- \\
4.874776\end{array}$ & 3.126100 & $\begin{array}{l}- \\
0.588709\end{array}$ \\
\hline 37. & 1. & 0. & $\begin{array}{l}- \\
6.546365\end{array}$ & 2.641859 & $\begin{array}{l}- \\
0.955820\end{array}$ \\
\hline 38. & 1. & 0. & $\begin{array}{l}- \\
2.797594\end{array}$ & 0.501986 & $\begin{array}{l}- \\
1.087089\end{array}$ \\
\hline 39. & 1. & 0. & $\begin{array}{l}- \\
1.966344\end{array}$ & 2.550740 & 1.040265 \\
\hline 40. & 1. & 0. & $\begin{array}{l}- \\
0.784844\end{array}$ & 1.662759 & \begin{tabular}{|l|}
- \\
1.671871 \\
\end{tabular} \\
\hline 41. & 1. & 0. & 0.244054 & 3.931571 & $\begin{array}{l}- \\
1.762756 \\
\end{array}$ \\
\hline 42. & 1. & 0. & $\begin{array}{l} \\
1.523568\end{array}$ & 4.073598 & $\begin{array}{l} \\
1.667816\end{array}$ \\
\hline
\end{tabular}




\begin{tabular}{|c|c|c|c|c|c|}
\hline 43. & 1. & 0. & $\begin{array}{l}- \\
0.533141 \\
\end{array}$ & 4.361192 & \begin{tabular}{|l|}
- \\
0.224845 \\
\end{tabular} \\
\hline 44. & 1. & 0. & $\begin{array}{l}- \\
2.501034 \\
\end{array}$ & $\begin{array}{l}- \\
0.243841\end{array}$ & 2.785060 \\
\hline 45. & 1. & 0. & $\begin{array}{l}- \\
2.781515 \\
\end{array}$ & $\begin{array}{l}- \\
1.962387\end{array}$ & 2.471940 \\
\hline 46. & 1. & 0. & $\begin{array}{l}- \\
1.918037\end{array}$ & $\begin{array}{l}- \\
0.969702\end{array}$ & 1.285845 \\
\hline 47. & 1. & 0. & \begin{tabular}{|l|} 
\\
4.962407 \\
\end{tabular} & 0.026932 & 3.236729 \\
\hline 48. & 1. & 0. & $\begin{array}{l}- \\
5.229260\end{array}$ & $\begin{array}{l}- \\
1.685776\end{array}$ & 2.888430 \\
\hline 49. & 1. & 0. & $\begin{array}{l} \\
6.176799\end{array}$ & $\begin{array}{l}- \\
0.457044\end{array}$ & 2.042803 \\
\hline 50. & 1. & 0. & 1.399200 & 0.941633 & $\begin{array}{l}- \\
1.609731\end{array}$ \\
\hline 51. & 1. & 0. & 1.340637 & $\begin{array}{l}- \\
1.363131\end{array}$ & $\begin{array}{l} \\
1.925299\end{array}$ \\
\hline 52. & 1. & 0. & 2.204089 & $\begin{array}{l}- \\
1.917231\end{array}$ & 0.960370 \\
\hline 53. & 1. & 0. & 3.653884 & $\begin{array}{l}- \\
0.337026\end{array}$ & $\begin{array}{l}- \\
1.217467\end{array}$ \\
\hline 54. & 1. & 0. & 2.256822 & 0.676445 & 1.319914 \\
\hline 55. & 1. & 0. & 2.689423 & 2.696998 & 0.201481 \\
\hline 56. & 1. & 0. & 3.942601 & $\begin{array}{l}- \\
2.950608\end{array}$ & $\begin{array}{l} \\
0.216906\end{array}$ \\
\hline 57. & 1. & 0. & 4.868828 & 1.852110 & 0.642344 \\
\hline 58. & 1. & 0. & 0.541917 & $\begin{array}{l}- \\
3.571416\end{array}$ & $\begin{array}{l}- \\
1.176124\end{array}$ \\
\hline 59. & 1. & 0. & $\begin{array}{l} \\
0.050695 \\
\end{array}$ & $\begin{array}{l}- \\
2.866703\end{array}$ & 0.344988 \\
\hline 60. & 1. & 0. & $\begin{array}{l} \\
1.313666 \\
\end{array}$ & $\begin{array}{l}- \\
1.461534\end{array}$ & $\begin{array}{l} \\
1.118437\end{array}$ \\
\hline & & & & & \\
\hline & & & & & \\
\hline
\end{tabular}

\begin{tabular}{|c|c|c|c|c|c|}
\hline \multicolumn{2}{|c|}{$3-6 R-2$} & \multicolumn{4}{|c|}{$\begin{array}{l}\text { Standard Orientation } \\
\text { (Ångstroms) }\end{array}$} \\
\hline $\begin{array}{l}\text { Center } \\
\text { number }\end{array}$ & $\begin{array}{c}\text { Atom } \\
\text { number }\end{array}$ & Type & $\mathrm{X}$ & Y & Z \\
\hline 1. & 6. & 0. & $-\overline{3.993925}$ & -0.077835 & 2.183105 \\
\hline
\end{tabular}




\begin{tabular}{|c|c|c|c|c|c|}
\hline 2. & 6. & 0. & 3.723998 & 1.557157 & 1.930933 \\
\hline 3. & 6. & 0. & $\begin{array}{l}- \\
3.556476\end{array}$ & $\begin{array}{l}- \\
1.979326\end{array}$ & 0.526429 \\
\hline 4. & 6. & 0. & $\begin{array}{l}- \\
3.703476\end{array}$ & $\begin{array}{l}- \\
1.140052\end{array}$ & $\begin{array}{l}- \\
0.520099\end{array}$ \\
\hline 5. & 6. & 0. & $\begin{array}{l}- \\
3.953205\end{array}$ & 0.352820 & $\begin{array}{l} \\
0.312759\end{array}$ \\
\hline 6. & 6. & 0. & $\begin{array}{l}- \\
4.710684 \\
\end{array}$ & 0.647705 & 1.023455 \\
\hline 7. & 6. & 0. & $\begin{array}{l}- \\
3.583461\end{array}$ & $\begin{array}{l}- \\
1.610494\end{array}$ & $\begin{array}{l}- \\
1.942990\end{array}$ \\
\hline 8. & 6. & 0. & $\begin{array}{l}- \\
2.604285\end{array}$ & 1.034508 & 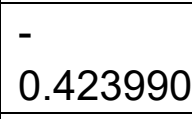 \\
\hline 9. & 6. & 0. & $\begin{array}{l}- \\
2.237516\end{array}$ & 1.860617 & $\begin{array}{l} \\
1.404543\end{array}$ \\
\hline 10. & 6. & 0. & $-\overline{0.869613}$ & 2.484208 & $\begin{array}{l}- \\
1.550974\end{array}$ \\
\hline 11. & 6. & 0. & $\begin{array}{l}- \\
0.905028\end{array}$ & 4.004173 & - \\
\hline 12. & 8. & 0. & 0.056877 & 1.963090 & $\begin{array}{l} \\
0.572315 \\
\end{array}$ \\
\hline 13. & 6. & 0. & $\begin{array}{l}- \\
4.748896\end{array}$ & 2.160125 & 1.301049 \\
\hline 14. & 6. & 0. & $-\overline{6.163234}$ & 0.139497 & 0.904082 \\
\hline 15. & 8. & 0. & $\begin{array}{l}- \\
3.630261\end{array}$ & $\begin{array}{l}- \\
2.355542\end{array}$ & 2.852506 \\
\hline 16. & 1. & 0. & $\begin{array}{l} \\
4.581023\end{array}$ & 0.712679 & $\begin{array}{l}- \\
1.139770 \\
\end{array}$ \\
\hline 17. & 6. & 0. & 0.952094 & 0.994924 & $\begin{array}{l}- \\
1.018267 \\
\end{array}$ \\
\hline 18. & 8. & 0. & 0.274914 & $\begin{array}{l}- \\
0.216037\end{array}$ & $\begin{array}{l} \\
1.289532 \\
\end{array}$ \\
\hline 19. & 6. & 0. & 1.138208 & - & $\begin{array}{l}- \\
1.763202 \\
\end{array}$ \\
\hline 20. & 6. & 0. & 2.198195 & - & $\begin{array}{l}- \\
0.698459\end{array}$ \\
\hline 21. & 6. & 0. & 2.943562 & $\begin{array}{l}- \\
0.308402\end{array}$ & $\begin{array}{l} \\
0.284740\end{array}$ \\
\hline 22. & 6. & 0. & 1.965535 & 0.790644 & 0.112311 \\
\hline 23. & 8. & 0. & 2.676640 & 2.012127 & 0.343934 \\
\hline 24. & 8. & 0. & 3.088379 & - & - \\
\hline
\end{tabular}




\begin{tabular}{|c|c|c|c|c|c|}
\hline & & & & 2.524024 & 1.264398 \\
\hline 25. & 8. & 0. & 3.788779 & $\begin{array}{l} \\
0.693474 \\
\end{array}$ & 0.830271 \\
\hline 26. & 16. & 0. & 5.223934 & 0.093566 & 1.057232 \\
\hline 27. & 8. & 0. & 5.988427 & $\begin{array}{l} \\
0.759422 \\
\end{array}$ & 1.933867 \\
\hline 28. & 8. & 0. & 5.714826 & 0.521011 & $\begin{array}{l}- \\
0.243899\end{array}$ \\
\hline 29. & 8. & 0. & 4.748171 & 1.359249 & 1.916034 \\
\hline 30. & 6. & 0. & 0.230092 & $\begin{array}{l} \\
2.449119 \\
\end{array}$ & $\begin{array}{l} \\
2.075247\end{array}$ \\
\hline 31. & 8. & 0. & $\begin{array}{l} \\
0.512948\end{array}$ & $\begin{array}{l} \\
2.869906 \\
\end{array}$ & $\begin{array}{l} \\
0.946529\end{array}$ \\
\hline 32. & 1. & 0. & $\begin{array}{l} \\
3.019787\end{array}$ & 0.394428 & 2.374073 \\
\hline 33. & 1. & 0. & $\begin{array}{l}- \\
4.560449\end{array}$ & 0.005328 & 3.116036 \\
\hline 34. & 1. & 0. & $\begin{array}{l}- \\
3.341545\end{array}$ & $\begin{array}{l}- \\
3.034398\end{array}$ & 0.375385 \\
\hline 35. & 1. & 0. & 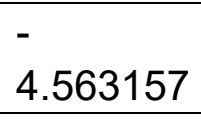 & $\begin{array}{l} \\
1.575119\end{array}$ & $\begin{array}{l}- \\
2.437371\end{array}$ \\
\hline 36. & 1. & 0. & $\begin{array}{l}- \\
2.918682\end{array}$ &  & $\begin{array}{l}- \\
2.512823\end{array}$ \\
\hline 37. & 1. & 0. & $\begin{array}{l}- \\
3.200469\end{array}$ & $\begin{array}{l} \\
2.631585\end{array}$ & $\begin{array}{l}- \\
2.001120\end{array}$ \\
\hline 38. & 1. & 0. & $-\overline{1.863660}$ & 0.767388 & 0.325101 \\
\hline 39. & 1. & 0. & $\begin{array}{l}- \\
2.950521\end{array}$ & 2.144807 & $\begin{array}{l}- \\
2.179030\end{array}$ \\
\hline 40. & 1. & 0. & $\begin{array}{l} \\
0.480190\end{array}$ & 2.246588 & $\begin{array}{l}- \\
2.552589\end{array}$ \\
\hline 41. & 1. & 0. & 0.082265 & 4.437243 & $\begin{array}{l}1.566301 \\
\end{array}$ \\
\hline 42. & 1. & 0. & $\begin{array}{l} \\
1.615719\end{array}$ & 4.453199 & $\begin{array}{l}- \\
2.080535 \\
\end{array}$ \\
\hline 43. & 1. & 0. & $\begin{array}{l}- \\
1.219721\end{array}$ & 4.258874 & $\begin{array}{l}- \\
0.364405\end{array}$ \\
\hline 44. & 1. & 0. & $\begin{array}{l}- \\
5.233882 \\
\end{array}$ & 2.699169 & 0.479102 \\
\hline 45. & 1. & 0. & $\begin{array}{l} \\
5.317606\end{array}$ & 2.364659 & 2.214418 \\
\hline 46. & 1. & 0. & $\begin{array}{l}- \\
3.746170\end{array}$ & 2.578337 & 1.427628 \\
\hline
\end{tabular}




\begin{tabular}{|c|c|c|c|c|c|}
\hline 47. & 1. & 0. & 6.693061 & 0.664246 & 0.101144 \\
\hline 48. & 1. & 0. & $\overline{6} .708339$ & 0.322404 & 1.835974 \\
\hline 49. & 1. & 0. & $-\overline{6.213187}$ & $-\overline{0.932652}$ & 0.695102 \\
\hline 50. & 1. & 0. & 1.472292 & 1.336754 & $\begin{array}{l}- \\
1.933050 \\
\end{array}$ \\
\hline 51. & 1. & 0. & 1.650171 & $\overline{-} .944729$ & $\begin{array}{l}- \\
2.686187\end{array}$ \\
\hline 52. & 1. & 0. & 1.679220 & $\begin{array}{l} \\
\end{array} .996419$ & 0.178209 \\
\hline 53. & 1. & 0. & 3.581091 & 0.026288 & $-\overline{1.109501}$ \\
\hline 54. & 1. & 0. & 1.414404 & 0.487737 & 1.012297 \\
\hline 55. & 1. & 0. & 2.008254 & 2.696984 & 0.499400 \\
\hline 56. & 1. & 0. & 3.716827 & $\begin{array}{l}- \\
2.780719\end{array}$ & $\begin{array}{l}- \\
0.575185\end{array}$ \\
\hline 57. & 1. & 0. & 4.032072 & 1.818115 & 1.399622 \\
\hline 58. & 1. & 0. & $\overline{-}-426845$ & $\begin{array}{l}- \\
2.170165\end{array}$ & $\begin{array}{l}- \\
2.912804\end{array}$ \\
\hline 59. & 1. & 0. & 0.850146 & $\begin{array}{l}- \\
3.292659\end{array}$ & $\begin{array}{l}- \\
2.387268\end{array}$ \\
\hline 60. & 1. & 0. & $-\overline{1.009433}$ & $\begin{array}{l}- \\
2.102971\end{array}$ & $\begin{array}{l} \\
0.624890\end{array}$ \\
\hline & & & & & \\
\hline
\end{tabular}

\begin{tabular}{|l|l|l|c|l|l|}
\hline \multicolumn{2}{|c|}{ 3-6R-3 } & \multicolumn{4}{c|}{$\begin{array}{c}\text { Standard Orientation } \\
\text { (Ångstroms) }\end{array}$} \\
\hline $\begin{array}{c}\text { Center } \\
\text { number }\end{array}$ & $\begin{array}{c}\text { Atom } \\
\text { number }\end{array}$ & Type & X & \multicolumn{1}{c|}{ Y } & \multicolumn{1}{c|}{ Z } \\
\hline & & & 5.739220 & 1.054208 & 0.217482 \\
\hline 1. & 6. & 0. & & - & \\
\hline 2. & 6. & 0. & 5.760790 & 0.924213 & 1.299515 \\
\hline & & & & - & \\
3. & 6. & 0. & 4.767340 & 0.014119 & 1.899769 \\
\hline 4. & 6. & 0. & 3.900366 & 0.719760 & 1.174214 \\
\hline & 6. & 0. & 3.875041 & 0.648396 & 0.347129 \\
\hline 5. & 6. & 0. & 5.263113 & 0.218333 & - \\
\hline 6. & 6. & & & & \\
\hline
\end{tabular}




\begin{tabular}{|c|c|c|c|c|c|}
\hline & & & & & 0.948150 \\
\hline 7. & 6. & 0. & 2.893537 & 1.625096 & 1.831411 \\
\hline 8. & 6. & 0. & 2.714600 & $\begin{array}{l}- \\
0.196442\end{array}$ & $\begin{array}{l}- \\
0.853243\end{array}$ \\
\hline 9. & 6. & 0. & 2.007079 & $\begin{array}{l}- \\
1.089421 \\
\end{array}$ & $\begin{array}{l}- \\
0.155352 \\
\end{array}$ \\
\hline 10. & 6. & 0. & 0.878005 & $\begin{array}{l}- \\
1.903792\end{array}$ & $\begin{array}{l}- \\
0.727866\end{array}$ \\
\hline 11. & 6. & 0. & 1.144772 & $\begin{array}{l}- \\
3.404031\end{array}$ & $\begin{array}{l}- \\
0.643090\end{array}$ \\
\hline 12. & 8. & 0. & $\begin{array}{l} \\
0.351015 \\
\end{array}$ & $\begin{array}{l} \\
1.675565 \\
\end{array}$ & 0.019530 \\
\hline 13. & 6. & 0. & 5.157676 & $\begin{array}{l} \\
0.065005 \\
\end{array}$ & $\begin{array}{l} \\
2.457400 \\
\end{array}$ \\
\hline 14. & 6. & 0. & 6.278229 & 1.363745 & $\begin{array}{l}- \\
0.747891\end{array}$ \\
\hline 15. & 8. & 0. & 6.541761 & $\begin{array}{l}- \\
1.563933 \\
\end{array}$ & 1.990517 \\
\hline 16. & 1. & 0. & 3.682875 & 1.667423 & 0.717505 \\
\hline 17. & 6. & 0. & $\begin{array}{l}- \\
1.133701\end{array}$ & $-\overline{0.602518}$ & $-\overline{0.383878}$ \\
\hline 18. & 8. & 0. & $\begin{array}{l}- \\
0.571754\end{array}$ & 0.625780 & 0.044705 \\
\hline 19. & 6. & 0. & $\begin{array}{l}- \\
1.323615\end{array}$ & 1.763265 & $\begin{array}{l} \\
0.409852\end{array}$ \\
\hline 20. & 6. & 0. & $\begin{array}{l}- \\
2.713786\end{array}$ & 1.733385 & 0.237877 \\
\hline 21. & 6. & 0. & $\begin{array}{l}- \\
3.400974\end{array}$ & 0.397266 & $\begin{array}{l}- \\
0.083110\end{array}$ \\
\hline 22. & 6. & 0. & $\begin{array}{l}- \\
2.504607\end{array}$ & $\begin{array}{l}- \\
0.782956\end{array}$ & 0.279008 \\
\hline 23. & 8. & 0. & $\begin{array}{l}- \\
3.103241\end{array}$ & $\begin{array}{l}- \\
2.000591\end{array}$ & $\begin{array}{l}- \\
0.178531\end{array}$ \\
\hline 24. & 8. & 0. & $\begin{array}{l} \\
3.453540\end{array}$ & 2.823529 & $\begin{array}{l} \\
0.266606\end{array}$ \\
\hline 25. & 8. & 0. & 4.624310 & 0.395116 & 0.696926 \\
\hline 26. & 16. & 0. & $\begin{array}{l}- \\
5.943429 \\
\end{array}$ & $\begin{array}{l}- \\
0.419752 \\
\end{array}$ & 0.118847 \\
\hline 27. & 8. & 0. & 7.060634 & 0.093020 & 0.873396 \\
\hline 28. & 8. & 0. & - & - & - \\
\hline
\end{tabular}




\begin{tabular}{|c|c|c|c|c|c|}
\hline & & & 5.893935 & 0.379654 & 1.334346 \\
\hline 29. & 8. & 0. & $\begin{array}{l}- \\
5.646180\end{array}$ & $\begin{array}{l}- \\
1.901079\end{array}$ & 0.647403 \\
\hline 30. & 6. & 0. & $-\overline{0.484653}$ & 2.982448 & $-\overline{0.046017}$ \\
\hline 31. & 8. & 0. & 0.797185 & 2.899302 & $\begin{array}{l}- \\
0.644785\end{array}$ \\
\hline 32. & 1. & 0. & 5.060322 & $\begin{array}{l} \\
1.885606 \\
\end{array}$ & $\begin{array}{l}- \\
0.455283\end{array}$ \\
\hline 33. & 1. & 0. & 6.737687 & $\begin{array}{l}- \\
1.358190\end{array}$ & 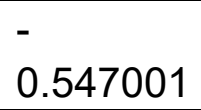 \\
\hline 34. & 1. & 0. & 4.784016 & 0.045998 & 2.985321 \\
\hline 35. & 1. & 0. & 2.923561 & 2.631806 & 1.398967 \\
\hline 36. & 1. & 0. & 1.874592 & 1.249705 & 1.675879 \\
\hline 37. & 1. & 0. & 3.064983 & 1.698102 & 2.907986 \\
\hline 38. & 1. & 0. & 2.446146 & $\begin{array}{l}- \\
0.027022\end{array}$ & $\begin{array}{l}- \\
1.895871\end{array}$ \\
\hline 39. & 1. & 0. & 2.235123 & $\begin{array}{l}- \\
1.281253 \\
\end{array}$ & 0.892026 \\
\hline 40. & 1. & 0. & 0.702393 & $\begin{array}{l} \\
1.612741\end{array}$ & $\begin{array}{l}-773318 \\
\end{array}$ \\
\hline 41. & 1. & 0. & 0.307599 & $\begin{array}{l}- \\
3.968481\end{array}$ & $\begin{array}{l} \\
1.064183\end{array}$ \\
\hline 42. & 1. & 0. & 2.051827 & $\begin{array}{l}- \\
3.658919\end{array}$ & $\begin{array}{l} \\
1.197530\end{array}$ \\
\hline 43. & 1. & 0. & 1.281404 & $\begin{array}{l}- \\
3.709038\end{array}$ & 0.398763 \\
\hline 44. & 1. & 0. & 4.755727 & 0.799575 & $\begin{array}{l}- \\
2.998653\end{array}$ \\
\hline 45. & 1. & 0. & 6.150308 & $\begin{array}{l}- \\
0.277311\end{array}$ & $\begin{array}{l}- \\
2.867934\end{array}$ \\
\hline 46. & 1. & 0. & 4.520792 & $\begin{array}{l}- \\
0.928149\end{array}$ & $\begin{array}{l}- \\
2.672026\end{array}$ \\
\hline 47. & 1. & 0. & 5.967639 & 2.257006 & $\begin{array}{l}- \\
1.300986\end{array}$ \\
\hline 48. & 1. & 0. & 7.263863 & 1.067413 & $\begin{array}{l}- \\
1.121612\end{array}$ \\
\hline 49. & 1. & 0. & 6.389219 & 1.641318 & 0.303265 \\
\hline 50. & 1. & 0. & 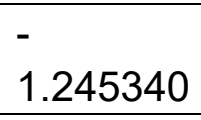 & $\begin{array}{l}- \\
0.589935\end{array}$ & $-\overline{1.484330}$ \\
\hline 51. & 1. & 0. & $\begin{array}{l}- \\
1.433200\end{array}$ & 1.721080 & $\begin{array}{l}- \\
1.504770\end{array}$ \\
\hline 52. & 1. & 0. & - & 1.800722 & 1.330491 \\
\hline
\end{tabular}




\begin{tabular}{|c|c|c|c|c|c|}
\hline & & & 2.589810 & & \\
\hline 53. & 1. & 0. & $\begin{array}{l}- \\
3.661111\end{array}$ & 0.367281 & $\begin{array}{l}- \\
1.146223\end{array}$ \\
\hline 54. & 1. & 0. & $\begin{array}{l}- \\
2.351005\end{array}$ & $\begin{array}{l}- \\
0.811900\end{array}$ & 1.365666 \\
\hline 55. & 1. & 0. & $\begin{array}{l} \\
2.452995\end{array}$ & $\begin{array}{l}- \\
2.702525\end{array}$ & $\begin{array}{l}- \\
0.020482\end{array}$ \\
\hline 56. & 1. & 0. & $\begin{array}{l}- \\
4.337761\end{array}$ & 2.781731 & 0.125091 \\
\hline 57. & 1. & 0. & $\begin{array}{l}- \\
4.747494 \\
\end{array}$ & $\begin{array}{l}- \\
2.165118 \\
\end{array}$ & 0.309451 \\
\hline 58. & 1. & 0. & $\begin{array}{l}- \\
0.972682 \\
\end{array}$ & 3.885628 & $\begin{array}{l}- \\
0.418132\end{array}$ \\
\hline 59. & 1. & 0. & $\begin{array}{l}- \\
0.417383 \\
\end{array}$ & 3.054568 & 1.051435 \\
\hline 60. & 1. & 0. & 1.118279 & 2.005523 & $\begin{array}{l}- \\
0.453737 \\
\end{array}$ \\
\hline & & & & & \\
\hline & & & & & \\
\hline
\end{tabular}

\begin{tabular}{|c|c|c|c|c|c|}
\hline \multicolumn{2}{|c|}{$3-6 R-4$} & \multicolumn{4}{|c|}{$\begin{array}{l}\text { Standard Orientation } \\
\text { (Angstroms) }\end{array}$} \\
\hline $\begin{array}{l}\text { Center } \\
\text { number }\end{array}$ & $\begin{array}{l}\text { Atom } \\
\text { number }\end{array}$ & Type & $\mathrm{X}$ & Y & Z \\
\hline 1. & 6. & 0. & 3.070718 & 0.894604 & $-\overline{1.203025}$ \\
\hline 2. & 6. & 0. & 2.337022 & $\begin{array}{l}- \\
1.802217\end{array}$ & $-\overline{0.242436}$ \\
\hline 3. & 6. & 0. & 2.317015 & $\begin{array}{l}- \\
1.447358\end{array}$ & 1.173918 \\
\hline 4. & 6. & 0. & 2.874758 & $\begin{array}{l}- \\
0.308545\end{array}$ & 1.640773 \\
\hline 5. & 6. & 0. & 3.496683 & 0.715390 & 0.705576 \\
\hline 6. & 6. & 0. & 4.124346 & 0.031340 & $\begin{array}{l}- \\
0.562688\end{array}$ \\
\hline 7. & 6. & 0. & 2.879703 & 0.021625 & 3.105380 \\
\hline 8. & 6. & 0. & 2.519586 & 1.842478 & 0.381579 \\
\hline 9. & 6. & 0. & 1.215505 & 1.871228 & 0.677693 \\
\hline 10. & 6. & 0. & 0.241229 & 2.992065 & 0.344856 \\
\hline 11. & 6. & 0. & 0.859213 & 4.384951 & 0.306423 \\
\hline 12. & 8. & 0. & $\begin{array}{l}- \\
0.378905\end{array}$ & 2.829257 & $\begin{array}{l}- \\
0.967971\end{array}$ \\
\hline
\end{tabular}




\begin{tabular}{|c|c|c|c|c|c|}
\hline 13. & 6. & 0. & 4.581606 & 1.074402 & 1.598126 \\
\hline 14. & 6. & 0. & 5.360762 & $\begin{array}{l}- \\
0.787127\end{array}$ & $\begin{array}{l}- \\
0.131960\end{array}$ \\
\hline 15. & 8. & 0. & 1.751788 & $\begin{array}{l}- \\
2.801232\end{array}$ & $\begin{array}{l}- \\
0.684936\end{array}$ \\
\hline 16. & 1. & 0. & 4.331737 & 1.191775 & \begin{tabular}{|l|}
1.243077 \\
\end{tabular} \\
\hline 17. & 6. & 0. & $\begin{array}{l}- \\
1.444191\end{array}$ & 1.927775 & $\begin{array}{l}- \\
1.123567 \\
\end{array}$ \\
\hline 18. & 8. & 0. & $\begin{array}{l}- \\
2.440062\end{array}$ & 2.142682 & $\begin{array}{l}- \\
0.144519\end{array}$ \\
\hline 19. & 6. & 0. & $\begin{array}{l} \\
3.612670\end{array}$ & 1.337491 & $\begin{array}{l} \\
0.361014\end{array}$ \\
\hline 20. & 6. & 0. & $\begin{array}{l} \\
3.254890\end{array}$ & $\begin{array}{l} \\
0.155089\end{array}$ & $\begin{array}{l} \\
0.253948 \\
\end{array}$ \\
\hline 21. & 6. & 0. & $\begin{array}{l}- \\
2.124428\end{array}$ & $\begin{array}{l}- \\
0.490384\end{array}$ & \begin{tabular}{|l|}
- \\
1.242820 \\
\end{tabular} \\
\hline 22. & 6. & 0. & $\begin{array}{l}- \\
0.953691\end{array}$ & 0.471501 & $\begin{array}{l} \\
1.114339 \\
\end{array}$ \\
\hline 23. & 8. & 0. & $\begin{array}{l}- \\
0.051184\end{array}$ & 0.290124 & $\begin{array}{l} \\
2.198031 \\
\end{array}$ \\
\hline 24. & 8. & 0. & $\begin{array}{l} \\
4.418698\end{array}$ & $\begin{array}{l}- \\
0.905067\end{array}$ & $\begin{array}{l} \\
0.536961\end{array}$ \\
\hline 25. & 8. & 0. & $\begin{array}{l} \\
1.650607\end{array}$ & $\begin{array}{l} \\
1.875817\end{array}$ & $\begin{array}{l} \\
1.148229 \\
\end{array}$ \\
\hline 26. & 16. & 0. & $\begin{array}{l} \\
1.510505\end{array}$ & $\begin{array}{l}- \\
2.589140\end{array}$ & 0.311069 \\
\hline 27. & 8. & 0. & $\begin{array}{l}- \\
0.829192\end{array}$ & $\begin{array}{l}- \\
1.680357\end{array}$ & 1.235010 \\
\hline 28. & 8. & 0. & $\begin{array}{l} \\
2.808182\end{array}$ & $\begin{array}{l} \\
3.140413 \\
\end{array}$ & 0.678900 \\
\hline 29. & 8. & 0. & 0.565746 & $\begin{array}{l} \\
3.778746 \\
\end{array}$ & $\begin{array}{l} \\
0.095563 \\
\end{array}$ \\
\hline 30. & 6. & 0. & $\begin{array}{l}- \\
4.625505\end{array}$ & 1.768323 & 0.696207 \\
\hline 31. & 8. & 0. & $\begin{array}{l} \\
4.122338\end{array}$ & 1.604084 & 2.010640 \\
\hline 32. & 1. & 0. & 2.274511 & $\begin{array}{l}- \\
0.308771\end{array}$ & $\begin{array}{l}- \\
1.681727 \\
\end{array}$ \\
\hline 33. & 1. & 0. & 3.505491 & $\begin{array}{l}- \\
1.515593\end{array}$ & \begin{tabular}{|l|}
- \\
1.993057 \\
\end{tabular} \\
\hline 34. & 1. & 0. & 1.819017 & $\begin{array}{l}- \\
2.142325 \\
\end{array}$ & 1.842360 \\
\hline
\end{tabular}




\begin{tabular}{|c|c|c|c|c|c|}
\hline 35. & 1. & 0. & 3.910276 & 0.029579 & 3.483970 \\
\hline 36. & 1. & 0. & 2.475932 & 1.025185 & 3.279110 \\
\hline 37. & 1. & 0. & 2.301708 & 0.697130 & 3.689962 \\
\hline 38. & 1. & 0. & 2.972161 & 2.697678 & $\overline{0}-115360$ \\
\hline 39. & 1. & 0. & 0.783684 & 1.022663 & 1.202653 \\
\hline 40. & 1. & 0. & 0.559273 & 2.976874 & 1.090456 \\
\hline 41. & 1. & 0. & 0.081062 & 5.126098 & 0.110501 \\
\hline 42. & 1. & 0. & 1.340930 & 4.618702 & 1.259742 \\
\hline 43. & 1. & 0. & 1.604092 & 4.463469 & $\begin{array}{l}- \\
0.490748\end{array}$ \\
\hline 44. & 1. & 0. & 5.289692 & 1.786827 & $\begin{array}{l}- \\
1.159149 \\
\end{array}$ \\
\hline 45. & 1. & 0. & 5.088785 & 0.577845 & 2.431851 \\
\hline 46. & 1. & 0. & 3.743003 & 1.639814 & 2.013890 \\
\hline 47. & 1. & 0. & 6.128722 & 0.135212 & 0.298884 \\
\hline 48. & 1. & 0. & 5.802189 & $\begin{array}{l}- \\
1.291669\end{array}$ & $\begin{array}{l}- \\
0.997567 \\
\end{array}$ \\
\hline 49. & 1. & 0. & 5.114683 & $\begin{array}{l}- \\
1.551953 \\
\end{array}$ & 0.609470 \\
\hline 50. & 1. & 0. & $\begin{array}{l}- \\
1.846093\end{array}$ & 2.156015 & $\begin{array}{l}- \\
2.124077\end{array}$ \\
\hline 51. & 1. & 0. & 4.025423 & 1.541524 & 1.362375 \\
\hline 52. & 1. & 0. & $\begin{array}{l}- \\
2.915842\end{array}$ & $\begin{array}{l}- \\
0.314896\end{array}$ & 0.776027 \\
\hline 53. & 1. & 0. & $\begin{array}{l}- \\
2.528053\end{array}$ & $\begin{array}{l}- \\
0.457224\end{array}$ & $\begin{array}{l}- \\
2.259092\end{array}$ \\
\hline 54. & 1. & 0. & $\overline{-}-469991$ & 0.269357 & $\begin{array}{l}- \\
0.156345\end{array}$ \\
\hline 55. & 1. & 0. & 0.497377 & 1.089759 & $\begin{array}{l}- \\
2.207205\end{array}$ \\
\hline 56. & 1. & 0. & $\begin{array}{l}- \\
4.284387\end{array}$ & $\begin{array}{l}- \\
1.788910\end{array}$ & $\begin{array}{l}- \\
0.157866\end{array}$ \\
\hline 57. & 1. & 0. & 0.382262 & $\begin{array}{l}- \\
3.435134\end{array}$ & $\begin{array}{l}- \\
0.249197\end{array}$ \\
\hline 58. & 1. & 0. & 4.912732 & 2.812403 & 0.500705 \\
\hline
\end{tabular}




\begin{tabular}{|c|c|c|c|c|c|}
\hline 59. & 1. & 0. & $\begin{array}{l}- \\
5.512637 \\
\end{array}$ & 1.137778 & 0.608593 \\
\hline 60. & 1. & 0. & \begin{tabular}{|l|}
- \\
3.296422 \\
\end{tabular} & 2.106135 & 2.049258 \\
\hline & & & & & \\
\hline
\end{tabular}

\begin{tabular}{|c|c|c|c|c|c|}
\hline \multicolumn{2}{|c|}{$3-6 R-5$} & \multicolumn{4}{|c|}{$\begin{array}{l}\text { Standard Orientation } \\
\text { (Ångstroms) }\end{array}$} \\
\hline $\begin{array}{l}\text { Center } \\
\text { number }\end{array}$ & $\begin{array}{l}\text { Atom } \\
\text { number }\end{array}$ & Type & $\mathrm{X}$ & Y & Z \\
\hline 1. & 6. & 0. & $\begin{array}{l}- \\
3.128016\end{array}$ & $\begin{array}{l}- \\
0.998009\end{array}$ & 1.482784 \\
\hline 2. & 6. & 0. & $\begin{array}{l}- \\
3.239097\end{array}$ & $\begin{array}{l}- \\
2.026206\end{array}$ & 0.368130 \\
\hline 3. & 6. & 0. & $\begin{array}{l}- \\
4.085042\end{array}$ & $\begin{array}{l}- \\
1.679741\end{array}$ & $\begin{array}{l}- \\
0.785299\end{array}$ \\
\hline 4. & 6. & 0. & $\begin{array}{l}- \\
4.734454\end{array}$ & $\begin{array}{l}- \\
0.502596\end{array}$ & $\begin{array}{l}- \\
0.892554\end{array}$ \\
\hline 5. & 6. & 0. & 4.626416 & 0.568845 & 0.179658 \\
\hline 6. & 6. & 0. & 4.335231 & $\overline{0} .043239$ & 1.599027 \\
\hline 7. & 6. & 0. & $\begin{array}{l}- \\
5.576166\end{array}$ & $\begin{array}{l}- \\
0.165740\end{array}$ & $\begin{array}{l}- \\
2.090852\end{array}$ \\
\hline 8. & 6. & 0. & $\begin{array}{l}- \\
3.614732\end{array}$ & 1.643049 & $\begin{array}{l} \\
0.208079\end{array}$ \\
\hline 9. & 6. & 0. & $\begin{array}{l}- \\
2.497731\end{array}$ & 1.437528 & $\begin{array}{l}- \\
0.909988 \\
\end{array}$ \\
\hline 10. & 6. & 0. & $\begin{array}{l} \\
1.406626\end{array}$ & 2.453897 & $\begin{array}{l} \\
1.162841 \\
\end{array}$ \\
\hline 11. & 6. & 0. & $\begin{array}{l} \\
1.780386\end{array}$ & 3.909112 & $\begin{array}{l} \\
0.917614\end{array}$ \\
\hline 12. & 8. & 0. & $\begin{array}{l}- \\
0.282780\end{array}$ & 2.172166 & $\begin{array}{l}- \\
0.281451\end{array}$ \\
\hline 13. & 6. & 0. & $-\overline{4}-017491$ & 1.060419 & 2.623638 \\
\hline 14. & 6. & 0. & $\begin{array}{l}- \\
5.582294\end{array}$ & $\begin{array}{l}- \\
0.809079\end{array}$ & 2.088821 \\
\hline 15. & 8. & 0. & $\begin{array}{l}- \\
2.609226\end{array}$ & $\begin{array}{l}- \\
3.079926\end{array}$ & 0.403017 \\
\hline 16. & 1. & 0. & - & 1.066811 & 0.244016 \\
\hline
\end{tabular}




\begin{tabular}{|c|c|c|c|c|c|}
\hline & & & 5.604663 & & \\
\hline 17. & 6. & 0. & 0.653302 & 1.242700 & $\begin{array}{l}- \\
0.707925\end{array}$ \\
\hline 18. & 8. & 0. & 0.121386 & $\begin{array}{l}- \\
0.075982\end{array}$ & $\begin{array}{l}- \\
0.567842\end{array}$ \\
\hline 19. & 6. & 0. & 1.034960 & $\begin{array}{l}- \\
1.086469\end{array}$ & $\begin{array}{l}- \\
0.985490\end{array}$ \\
\hline 20. & 6. & 0. & 2.305811 & $\begin{array}{l}- \\
1.047768\end{array}$ & $\begin{array}{l}- \\
0.114505\end{array}$ \\
\hline 21. & 6. & 0. & 2.925842 & 0.352207 & $\begin{array}{l}- \\
0.117232\end{array}$ \\
\hline 22. & 6. & 0. & 1.888219 & 1.427845 & 0.187996 \\
\hline 23. & 8. & 0. & 2.476712 & 2.688212 & $\begin{array}{l}- \\
0.042183 \\
\end{array}$ \\
\hline 24. & 8. & 0. & 3.282297 & $\begin{array}{l}- \\
1.961008\end{array}$ & $\begin{array}{l}- \\
0.622748 \\
\end{array}$ \\
\hline 25. & 8. & 0. & 3.938175 & 0.439671 & 0.912677 \\
\hline 26. & 16. & 0. & 5.506890 & 0.132927 & 0.514880 \\
\hline 27. & 8. & 0. & 5.711633 & 0.508286 & $\begin{array}{l}- \\
0.876320\end{array}$ \\
\hline 28. & 8. & 0. & 6.293482 & 0.689828 & \begin{tabular}{|l|l}
1.589099 \\
\end{tabular} \\
\hline 29. & 8. & 0. & 5.552858 & $\begin{array}{l}- \\
1.462121\end{array}$ & 0.669948 \\
\hline 30. & 6. & 0. & 0.266038 & $\begin{array}{l}- \\
2.400555 \\
\end{array}$ & $\begin{array}{l}- \\
0.859083 \\
\end{array}$ \\
\hline 31. & 8. & 0. & 1.156062 & $\begin{array}{l}- \\
3.475012\end{array}$ & $\begin{array}{l} \\
1.192717 \\
\end{array}$ \\
\hline 32. & 1. & 0. & $\begin{array}{l}- \\
2.217132\end{array}$ & $\begin{array}{l}- \\
0.419596\end{array}$ & 1.274399 \\
\hline 33. & 1. & 0. & $\begin{array}{l}- \\
2.951728\end{array}$ & $-\overline{1.533374}$ & 2.421511 \\
\hline 34. & 1. & 0. & $\begin{array}{l}- \\
4.152970\end{array}$ & $\begin{array}{l}- \\
2.434279\end{array}$ & $\begin{array}{l} \\
1.565378 \\
\end{array}$ \\
\hline 35. & 1. & 0. & $\begin{array}{l}- \\
6.622668\end{array}$ & 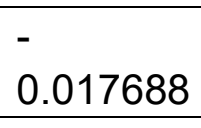 & $\begin{array}{l} \\
1.794949 \\
\end{array}$ \\
\hline 36. & 1. & 0. & $\begin{array}{l}- \\
5.241559\end{array}$ & 0.775586 & $\begin{array}{l}- \\
2.542115\end{array}$ \\
\hline 37. & 1. & 0. & $\begin{array}{l}- \\
5.540392 \\
\end{array}$ & $\begin{array}{l}- \\
0.950199\end{array}$ & $\begin{array}{l}- \\
2.850365 \\
\end{array}$ \\
\hline 38. & 1. & 0. & $\begin{array}{l}- \\
3.828625\end{array}$ & 2.634959 & 0.183369 \\
\hline 39. & 1. & 0. & $\begin{array}{l}- \\
2.279716\end{array}$ & 0.443264 & $\begin{array}{l} \\
1.288722\end{array}$ \\
\hline
\end{tabular}




\begin{tabular}{|c|c|c|c|c|c|}
\hline 40. & 1. & 0. & $\begin{array}{l}- \\
1.051236\end{array}$ & 2.342216 & $\begin{array}{l} \\
2.198241 \\
\end{array}$ \\
\hline 41. & 1. & 0. & $\begin{array}{l}- \\
0.932340\end{array}$ & 4.555317 & $\begin{array}{l}- \\
1.157676\end{array}$ \\
\hline 42. & 1. & 0. & $\begin{array}{l}- \\
2.628401\end{array}$ & 4.201775 & $\begin{array}{l}- \\
1.542633 \\
\end{array}$ \\
\hline 43. & 1. & 0. & $\begin{array}{l}- \\
2.044602\end{array}$ & 4.072486 & 0.130809 \\
\hline 44. & 1. & 0. & $\begin{array}{l}- \\
4.839179\end{array}$ & 1.782677 & 2.695926 \\
\hline 45. & 1. & 0. & $\begin{array}{l}- \\
3.877308\end{array}$ & 0.621349 & 3.617063 \\
\hline 46. & 1. & 0. & $\begin{array}{l}- \\
3.104106\end{array}$ & 1.604920 & 2.368939 \\
\hline 47. & 1. & 0. & $\begin{array}{l}- \\
6.435382\end{array}$ & $\begin{array}{l}- \\
0.130279\end{array}$ & 2.199586 \\
\hline 48. & 1. & 0. & $\begin{array}{l}- \\
5.391007\end{array}$ & 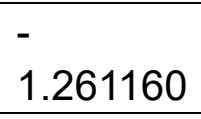 & 3.067656 \\
\hline 49. & 1. & 0. & $\begin{array}{l} \\
5.876073 \\
\end{array}$ & $\begin{array}{l} \\
1.608906 \\
\end{array}$ & 1.403953 \\
\hline 50. & 1. & 0. & 0.927130 & 1.409740 & $\begin{array}{l} \\
1.766337 \\
\end{array}$ \\
\hline 51. & 1. & 0. & 1.335774 & $\begin{array}{l}- \\
0.932149\end{array}$ & $\begin{array}{l}- \\
2.034774 \\
\end{array}$ \\
\hline 52. & 1. & 0. & 2.034017 & $\begin{array}{l}- \\
1.306207\end{array}$ & 0.919901 \\
\hline 53. & 1. & 0. & 3.387247 & 0.555223 & $\begin{array}{l} \\
1.089295 \\
\end{array}$ \\
\hline 54. & 1. & 0. & 1.550949 & 1.314058 & 1.229351 \\
\hline 55. & 1. & 0. & 1.807694 & 3.347991 & 0.187373 \\
\hline 56. & 1. & 0. & 2.815334 & $\begin{array}{l}- \\
2.804824\end{array}$ & $\begin{array}{l}- \\
0.785454\end{array}$ \\
\hline 57. & 1. & 0. & 4.797212 & $\begin{array}{l} \\
1.834433 \\
\end{array}$ & 0.133998 \\
\hline 58. & 1. & 0. & $\begin{array}{l}- \\
0.114701\end{array}$ & $\begin{array}{l}- \\
2.501784\end{array}$ & 0.163302 \\
\hline 59. & 1. & 0. & $\begin{array}{l}- \\
0.597107\end{array}$ & $\begin{array}{l}- \\
2.385171\end{array}$ & \begin{tabular}{|l|}
- \\
1.533920 \\
\end{tabular} \\
\hline 60. & 1. & 0. & 0.737555 & - & $\begin{array}{l}- \\
0.932786 \\
\end{array}$ \\
\hline & & & & & \\
\hline
\end{tabular}




\section{S1. ECD Calculation Details}

\section{ECD calculation details}

\section{Methods}

Monte Carlo conformational searches were carried out by means of the Spartan's 10 software using Merck Molecular Force Field (MMFF). The conformers with Boltzmann-population of over 5\% were chosen for ECD calculations, and then the conformers were initially optimized at B3LYP/6-31g (d, p) level in MeOH using the CPCM polarizable conductor calculation model. The theoretical calculation of ECD was conducted in $\mathrm{MeOH}$ using Time-dependent Density functional theory (TD-DFT) at the B3LYP/6-31+g (d, p) level for all conformers of compounds 3-6S. Rotatory strengths for a total of 30 excited states were calculated. ECD spectra were generated using the program SpecDis 1.6 (University of Würzburg, Würzburg, Germany) and GraphPad Prism 5 (University of California San Diego, USA) from dipole-length rotational strengths by applying Gaussian band shapes with sigma $=0.3 \mathrm{eV}$.

\section{Results}

Table S1.2.1. Gibbs free energies ${ }^{a}$ and equilibrium populations ${ }^{b}$ of low-energy conformers of 3-6S.

\begin{tabular}{|c|c|c|}
\hline \multirow{2}{*}{ Conformers } & \multicolumn{2}{|c|}{ In $\mathrm{MeOH}$} \\
\cline { 2 - 3 } & $\Delta G$ & $P(\%) / 100$ \\
\hline $3-6 S-1$ & 0.00 & 0.491 \\
\hline $3-6 S-2$ & 0.77 & 0.134 \\
\hline $3-6 S-3$ & 0.79 & 0.129 \\
\hline $3-6 S-4$ & 1.23 & 0.061 \\
\hline $3-6 S-5$ & 1.23 & 0.061 \\
\hline
\end{tabular}

${ }^{a} \mathrm{~B} 3 \mathrm{LYP} / 6-31+\mathrm{G}(\mathrm{d}, \mathrm{p})$, in $\mathrm{kcal} / \mathrm{mol} .{ }^{b}$ From $\Delta G$ values at $298.15 \mathrm{~K}$.

Table S1.2.2. Cartesian coordinates for the low-energy reoptimized MMFF conformers of 3-6S at B3LYP/6-311+G(d,p) level of theory in $\mathrm{CH}_{3} \mathrm{OH}$.

3-6S-1

Standard Orientation

(Ångstroms) 


\begin{tabular}{|c|c|c|c|c|c|}
\hline $\begin{array}{l}\text { Center } \\
\text { number }\end{array}$ & $\begin{array}{l}\text { Atomic } \\
\text { number }\end{array}$ & $\begin{array}{l}\text { Atomic } \\
\text { Type }\end{array}$ & $\mathrm{X}$ & $\mathrm{Y}$ & Z \\
\hline 1. & 6. & 0. & 1.844245 & $\begin{array}{l}- \\
1.482033\end{array}$ & 1.016928 \\
\hline 2. & 6. & 0. & 2.131696 & $\begin{array}{l}- \\
1.871418\end{array}$ & $\begin{array}{l}- \\
0.418438\end{array}$ \\
\hline 3. & 6. & 0. & 3.145014 & $\begin{array}{l}- \\
1.114392\end{array}$ & $\begin{array}{l}- \\
1.152843 \\
\end{array}$ \\
\hline 4. & 6. & 0. & 3.764384 & $\begin{array}{l}- \\
0.032679\end{array}$ & $\begin{array}{l}- \\
0.631964\end{array}$ \\
\hline 5. & 6. & 0. & 3.395078 & 0.500818 & \begin{tabular}{|l|l|}
0.743565 \\
\end{tabular} \\
\hline 6. & 6. & 0. & 2.960943 & $\begin{array}{l}- \\
0.665938 \\
\end{array}$ & 1.703238 \\
\hline 7. & 6. & 0. & 4.829748 & 0.709613 & $\begin{array}{l}- \\
1.386507 \\
\end{array}$ \\
\hline 8. & 6. & 0. & 2.367978 & 1.624057 & 0.652473 \\
\hline 9. & 6. & 0. & 1.783725 & 2.098506 & $\begin{array}{l}- \\
0.451503\end{array}$ \\
\hline 10. & 6. & 0. & 0.739544 & 3.196475 & $\begin{array}{l}- \\
0.480041\end{array}$ \\
\hline 11. & 6. & 0. & 1.340956 & 4.551650 & $\begin{array}{l}- \\
0.851403\end{array}$ \\
\hline 12. & 8. & 0. & 0.262481 & 2.941671 & $\begin{array}{l} \\
1.499980\end{array}$ \\
\hline 13. & 6. & 0. & 4.185434 & $\begin{array}{l}- \\
1.562259\end{array}$ & 1.991690 \\
\hline 14. & 6. & 0. & 2.442611 & $\begin{array}{l}- \\
0.135783\end{array}$ & 3.052765 \\
\hline 15. & 8. & 0. & 1.530135 & $\begin{array}{l}- \\
2.801534\end{array}$ & $\begin{array}{l} \\
0.972607\end{array}$ \\
\hline 16. & 1. & 0. & 4.301095 & 0.944450 & \begin{tabular}{|l|}
1.186104 \\
\end{tabular} \\
\hline 17. & 6. & 0. & $\begin{array}{l}- \\
1.330207\end{array}$ & 2.073746 & $\begin{array}{l} \\
1.214117 \\
\end{array}$ \\
\hline 18. & 8. & 0. & $\begin{array}{l}- \\
2.053101\end{array}$ & 2.527779 & $\begin{array}{l} \\
0.085697 \\
\end{array}$ \\
\hline 19. & 6. & 0. & $\begin{array}{l}- \\
3.240006\end{array}$ & 1.752670 & 0.166351 \\
\hline 20. & 6. & 0. & $\begin{array}{l}- \\
2.869185\end{array}$ & 0.297578 & 0.497831 \\
\hline 21. & 6. & 0. & $\begin{array}{l}- \\
1.973958\end{array}$ & $\begin{array}{l}- \\
0.294705\end{array}$ & $\begin{array}{l}-596498 \\
\end{array}$ \\
\hline 22. & 6. & 0. & 0.843717 & 0.637279 & 0.993868 \\
\hline
\end{tabular}




\begin{tabular}{|c|c|c|c|c|c|}
\hline 23. & 8. & 0. & $\begin{array}{l}- \\
0.213706\end{array}$ & 0.136100 & $\begin{array}{l}- \\
2.158749\end{array}$ \\
\hline 24. & 8. & 0. & $\begin{array}{l}- \\
4.079752\end{array}$ & $\begin{array}{l}- \\
0.420293\end{array}$ & 0.625456 \\
\hline 25. & 8. & 0. & $\begin{array}{l}- \\
1.305231\end{array}$ & $\begin{array}{l}- \\
1.477065\end{array}$ & $\begin{array}{l}- \\
0.063733\end{array}$ \\
\hline 26. & 16. & 0. & $\begin{array}{l}- \\
1.919300\end{array}$ & $\begin{array}{l}- \\
2.964705\end{array}$ & $\begin{array}{l}- \\
0.375114\end{array}$ \\
\hline 27. & 8. & 0. & $\begin{array}{l} \\
2.976251\end{array}$ & $\begin{array}{l}- \\
3.235415\end{array}$ & 0.594681 \\
\hline 28. & 8. & 0. & $\begin{array}{l}- \\
2.197995\end{array}$ & $\begin{array}{l}- \\
3.072583\end{array}$ & $\begin{array}{l}- \\
1.797218\end{array}$ \\
\hline 29. & 8. & 0. & $\begin{array}{l}- \\
0.640949\end{array}$ & $\begin{array}{l}- \\
3.778743\end{array}$ & 0.065150 \\
\hline 30. & 6. & 0. & 3.961445 & 2.448809 & 1.317570 \\
\hline 31. & 8. & 0. & $\begin{array}{l}- \\
3.130901\end{array}$ & 2.573184 & 2.460539 \\
\hline 32. & 1. & 0. & 1.616736 & $\begin{array}{l}- \\
2.394691\end{array}$ & 1.578472 \\
\hline 33. & 1. & 0. & 0.909999 & $\begin{array}{l}- \\
0.908172\end{array}$ & 0.997794 \\
\hline 34. & 1. & 0. & 3.374096 & $\begin{array}{l}- \\
1.474140\end{array}$ & $\begin{array}{l}- \\
2.152054\end{array}$ \\
\hline 35. & 1. & 0. & 4.940031 & 0.344136 & $\begin{array}{l} \\
2.410024 \\
\end{array}$ \\
\hline 36. & 1. & 0. & 4.615416 & 1.783767 & $\begin{array}{l} \\
1.413551 \\
\end{array}$ \\
\hline 37. & 1. & 0. & 5.794782 & 0.598164 & $\begin{array}{l} \\
0.874918\end{array}$ \\
\hline 38. & 1. & 0. & 2.100163 & 2.070824 & \begin{tabular}{|l|l|}
1.608930 \\
\end{tabular} \\
\hline 39. & 1. & 0. & 2.040759 & 1.687381 & $\begin{array}{l} \\
1.425970 \\
\end{array}$ \\
\hline 40. & 1. & 0. & 0.245891 & 3.268468 & 0.495237 \\
\hline 41. & 1. & 0. & 2.095484 & 4.845696 & $\begin{array}{l}- \\
0.116549 \\
\end{array}$ \\
\hline 42. & 1. & 0. & 1.815333 & 4.501904 & $\begin{array}{l} \\
1.836286 \\
\end{array}$ \\
\hline 43. & 1. & 0. & 0.557053 & 5.312606 & $\begin{array}{l} \\
0.885125 \\
\end{array}$ \\
\hline 44. & 1. & 0. & 4.619496 & $\begin{array}{l}- \\
1.985594\end{array}$ & 1.082222 \\
\hline 45. & 1. & 0. & 4.968245 & - & 2.505219 \\
\hline
\end{tabular}




\begin{tabular}{|c|c|c|c|c|c|}
\hline & & & & 0.993069 & \\
\hline 46. & 1. & 0. & 3.900654 & $\begin{array}{l}- \\
2.394228 \\
\end{array}$ & 2.643568 \\
\hline 47. & 1. & 0. & 1.505415 & 0.418458 & 2.954521 \\
\hline 48. & 1. & 0. & 2.253277 & $\begin{array}{l}- \\
0.972160\end{array}$ & 3.733182 \\
\hline 49. & 1. & 0. & 3.180626 & 0.517466 & 3.532370 \\
\hline 50. & 1. & 0. & $\begin{array}{l}- \\
1.965214\end{array}$ & 2.118903 & $\begin{array}{l} \\
2.112657\end{array}$ \\
\hline 51. & 1. & 0. & $\begin{array}{l}- \\
3.887285\end{array}$ & 1.759522 & $\begin{array}{l}- \\
0.725067\end{array}$ \\
\hline 52. & 1. & 0. & $\begin{array}{l}- \\
2.302045\end{array}$ & 0.309329 & 1.439822 \\
\hline 53. & 1. & 0. & $\begin{array}{l}- \\
2.566714\end{array}$ & $\begin{array}{l}- \\
0.575059\end{array}$ & $\begin{array}{l}- \\
1.472836\end{array}$ \\
\hline 54. & 1. & 0. & $\begin{array}{l}- \\
0.145216\end{array}$ & 0.666521 & $\begin{array}{l}- \\
0.149283\end{array}$ \\
\hline 55. & 1. & 0. & 0.261916 & 0.877662 & $\begin{array}{l} \\
2.559152\end{array}$ \\
\hline 56. & 1. & 0. & $\begin{array}{l}- \\
3.877601\end{array}$ & $\begin{array}{l}- \\
1.335807\end{array}$ & 0.884138 \\
\hline 57. & 1. & 0. & 0.208326 & $\begin{array}{l}- \\
3.426224\end{array}$ & $\begin{array}{l}- \\
0.384301\end{array}$ \\
\hline 58. & 1. & 0. & - & 3.430385 & 0.970075 \\
\hline 59. & 1. & 0. & $\begin{array}{l}- \\
4.824537\end{array}$ & 1.845820 & 1.607295 \\
\hline 60. & 1. & 0. & $\begin{array}{l}- \\
2.346006\end{array}$ & 3.056197 & 2.166518 \\
\hline & & & & & \\
\hline & & & & & \\
\hline
\end{tabular}

\begin{tabular}{|c|c|c|c|c|c|}
\hline \multicolumn{2}{|c|}{$3-6 S-2$} & \multicolumn{4}{|c|}{$\begin{array}{l}\text { Standard Orientation } \\
\text { (Ångstroms) }\end{array}$} \\
\hline $\begin{array}{l}\text { Center } \\
\text { number }\end{array}$ & $\begin{array}{l}\text { Atom } \\
\text { number }\end{array}$ & Type & $\mathrm{X}$ & Y & Z \\
\hline 1. & 6. & 0. & $\begin{array}{l}- \\
2.034142 \\
\end{array}$ & 1.209835 & 1.079760 \\
\hline 2. & 6. & 0. & $\begin{array}{l}- \\
2.413655\end{array}$ & 1.650804 & $\begin{array}{l}- \\
0.316229\end{array}$ \\
\hline 3. & 6. & 0. & $\begin{array}{l}- \\
3.376298\end{array}$ & 0.856206 & $\begin{array}{l}- \\
1.065597\end{array}$ \\
\hline
\end{tabular}




\begin{tabular}{|c|c|c|c|c|c|}
\hline 4. & 6. & 0. & 3.849968 & $\begin{array}{l} \\
0.323127\end{array}$ & 0.604908 \\
\hline 5. & 6. & 0. & $\begin{array}{l}- \\
3.385940\end{array}$ & $\begin{array}{l}- \\
0.896465 \\
\end{array}$ & 0.722535 \\
\hline 6. & 6. & 0. & \begin{tabular}{|l} 
\\
3.035595
\end{tabular} & 0.242443 & 1.745664 \\
\hline 7. & 6. & 0. & $\begin{array}{l}- \\
4.826945\end{array}$ & \begin{tabular}{|l|} 
\\
1.143693
\end{tabular} & $\begin{array}{l}- \\
1.394584\end{array}$ \\
\hline 8. & 6. & 0. & $\begin{array}{l}- \\
2.254445 \\
\end{array}$ & \begin{tabular}{|l|}
- \\
1.900334 \\
\end{tabular} & 0.530087 \\
\hline 9. & 6. & 0. & $\begin{array}{l}- \\
1.535896\end{array}$ & $\begin{array}{l}- \\
2.099347\end{array}$ & $\begin{array}{l}- \\
0.578438\end{array}$ \\
\hline 10. & 6. & 0. & $\begin{array}{l} \\
0.401111\end{array}$ & -7.096183 & $\begin{array}{l}- \\
0.695371\end{array}$ \\
\hline 11. & 6. & 0. & $\begin{array}{l} \\
0.879059\end{array}$ & $\begin{array}{l}- \\
4.444783\end{array}$ & $\begin{array}{l}- \\
1.236412\end{array}$ \\
\hline 12. & 8. & 0. & 0.587444 & $\begin{array}{l}- \\
2.645186\end{array}$ & $\begin{array}{l}- \\
1.645301\end{array}$ \\
\hline 13. & 6. & 0. & $\begin{array}{l}- \\
4.327631 \\
\end{array}$ & 0.991687 & 2.138647 \\
\hline 14. & 6. & 0. & $\begin{array}{l} \\
2.410069 \\
\end{array}$ & $\begin{array}{l}- \\
0.323571 \\
\end{array}$ & 3.033254 \\
\hline 15. & 8. & 0. & $\begin{array}{l}- \\
1.910265 \\
\end{array}$ & 2.665031 & $\overline{0} .830283$ \\
\hline 16. & 1. & 0. & $\begin{array}{l}- \\
4.229978 \\
\end{array}$ & $\begin{array}{l} \\
1.455599 \\
\end{array}$ & 1.155169 \\
\hline 17. & 6. & 0. & 1.540924 & $\begin{array}{l} \\
1.711324 \\
\end{array}$ & $\begin{array}{l}- \\
1.256008\end{array}$ \\
\hline 18. & 8. & 0. & 2.376984 & $\begin{array}{l}- \\
2.284355 \\
\end{array}$ & $\begin{array}{l}- \\
0.247748\end{array}$ \\
\hline 19. & 6. & 0. & 3.519918 & $\begin{array}{l}- \\
1.490546\end{array}$ & 0.090471 \\
\hline 20. & 6. & 0. & 3.076792 & $\begin{array}{l}- \\
0.135510\end{array}$ & 0.664548 \\
\hline 21. & 6. & 0. & 2.112580 & 0.535563 & $\begin{array}{l}- \\
0.308471\end{array}$ \\
\hline 22. & 6. & 0. & 0.969371 & $\begin{array}{l} \\
0.380365\end{array}$ & 0.725365 \\
\hline 23. & 8. & 0. & 0.093506 & 0.209455 & $\begin{array}{l}- \\
1.664624\end{array}$ \\
\hline 24. & 8. & 0. & 4.238459 & 0.650229 & 0.851628 \\
\hline 25. & 8. & 0. & 1.550765 & 1.696905 & 0.380602 \\
\hline 26. & 16. & 0. & 1.485822 & 3.126804 & - \\
\hline
\end{tabular}




\begin{tabular}{|c|c|c|c|c|c|}
\hline & & & & & 0.438715 \\
\hline 27. & 8. & 0. & 2.604326 & 3.940635 & $\overline{-} .007031$ \\
\hline 28. & 8. & 0. & 1.316657 & 2.802175 & $\begin{array}{l}- \\
1.860075\end{array}$ \\
\hline 29. & 8. & 0. & 0.166801 & 3.708502 & 0.176124 \\
\hline 30. & 6. & 0. & 4.323717 & $\begin{array}{l}- \\
2.313945\end{array}$ & 1.094897 \\
\hline 31. & 8. & 0. & 3.542339 & $\begin{array}{l}- \\
2.667478\end{array}$ & 2.224007 \\
\hline 32. & 1. & 0. & $\begin{array}{l}- \\
1.882644\end{array}$ & 2.104928 & 1.692941 \\
\hline 33. & 1. & 0. & $\begin{array}{l}-049669 \\
\end{array}$ & 0.735919 & 0.992112 \\
\hline 34. & 1. & 0. & $\begin{array}{l}- \\
3.668405\end{array}$ & 1.247704 & $\begin{array}{l} \\
2.035715\end{array}$ \\
\hline 35. & 1. & 0. & $\begin{array}{l}- \\
5.778552\end{array}$ & $\begin{array}{l}- \\
1.219734\end{array}$ & 0.852637 \\
\hline 36. & 1. & 0. & $\begin{array}{l}- \\
5.023052\end{array}$ & $\begin{array}{l}- \\
0.715396\end{array}$ & $\begin{array}{l}- \\
2.379954\end{array}$ \\
\hline 37. & 1. & 0. & $\begin{array}{l}- \\
4.455704\end{array}$ & $\begin{array}{l}- \\
2.166780\end{array}$ & $\begin{array}{l}- \\
1.520314\end{array}$ \\
\hline 38. & 1. & 0. & $\begin{array}{l}- \\
2.026687\end{array}$ & $\begin{array}{l}- \\
2.494624\end{array}$ & 1.414745 \\
\hline 39. & 1. & 0. & $\begin{array}{l}- \\
1.719842\end{array}$ & $\begin{array}{l}- \\
1.522284\end{array}$ & $\begin{array}{l}- \\
1.480918\end{array}$ \\
\hline 40. & 1. & 0. & 0.072765 & $\begin{array}{l}- \\
3.247212\end{array}$ & 0.282673 \\
\hline 41. & 1. & 0. & $\begin{array}{l}- \\
1.619819\end{array}$ & $\begin{array}{l}- \\
4.884285 \\
\end{array}$ & $\begin{array}{l}- \\
0.562709\end{array}$ \\
\hline 42. & 1. & 0. & $\begin{array}{l} \\
1.335754\end{array}$ & $\begin{array}{l} \\
4.316540\end{array}$ & $\begin{array}{l}- \\
2.222302\end{array}$ \\
\hline 43. & 1. & 0. & $\begin{array}{l}- \\
0.032242\end{array}$ & $\begin{array}{l} \\
5.129091\end{array}$ & $\begin{array}{l} \\
1.335220 \\
\end{array}$ \\
\hline 44. & 1. & 0. & 4.838993 & 1.426750 & 1.276004 \\
\hline 45. & 1. & 0. & $\begin{array}{l} \\
5.029037 \\
\end{array}$ & 0.315121 & 2.639026 \\
\hline 46. & 1. & 0. & 4.098909 & 1.804419 & 2.835693 \\
\hline 47. & 1. & 0. & $\begin{array}{l} \\
3.066861\end{array}$ & $\begin{array}{l}- \\
1.067800\end{array}$ & 3.497913 \\
\hline 48. & 1. & 0. & - & - & 2.853805 \\
\hline
\end{tabular}




\begin{tabular}{|c|c|c|c|c|c|}
\hline & & & 1.437832 & 0.790223 & \\
\hline 49. & 1. & 0. & $\begin{array}{l}- \\
2.257826\end{array}$ & 0.480255 & 3.760967 \\
\hline 50. & 1. & 0. & 2.132415 & $\begin{array}{l}- \\
1.522825\end{array}$ & $\begin{array}{l}- \\
2.165146\end{array}$ \\
\hline 51. & 1. & 0. & 4.135543 & $\begin{array}{l}- \\
1.306248\end{array}$ & $\begin{array}{l}- \\
0.805077\end{array}$ \\
\hline 52. & 1. & 0. & 2.558676 & $\begin{array}{l}- \\
0.324642\end{array}$ & 1.615411 \\
\hline 53. & 1. & 0. & 2.675544 & 0.873671 & $\begin{array}{l}- \\
1.185134\end{array}$ \\
\hline 54. & 1. & 0. & 0.397077 & $\begin{array}{l}- \\
0.617530\end{array}$ & 0.179404 \\
\hline 55. & 1. & 0. & 0.475740 & 1.038377 & $\begin{array}{l} \\
2.002670\end{array}$ \\
\hline 56. & 1. & 0. & 3.959242 & 1.520865 & 1.170681 \\
\hline 57. & 1. & 0. & $\begin{array}{l}- \\
0.692730\end{array}$ & 3.287409 & $\begin{array}{l}- \\
0.239731\end{array}$ \\
\hline 58. & 1. & 0. & 4.717997 & $\begin{array}{l}- \\
3.204326\end{array}$ & 0.583165 \\
\hline 59. & 1. & 0. & 5.164435 & $\begin{array}{l}- \\
1.716523\end{array}$ & 1.454909 \\
\hline 60. & 1. & 0. & 2.770526 & $\begin{array}{l}- \\
3.134011\end{array}$ & 1.873076 \\
\hline & & & & & \\
\hline
\end{tabular}

\begin{tabular}{|c|c|c|c|c|c|}
\hline \multicolumn{2}{|c|}{$3-6 S-3$} & \multicolumn{4}{|c|}{$\begin{array}{l}\text { Standard Orientation } \\
\text { (Ångstroms) }\end{array}$} \\
\hline $\begin{array}{l}\text { Center } \\
\text { number }\end{array}$ & $\begin{array}{l}\text { Atom } \\
\text { number }\end{array}$ & Type & $\mathrm{X}$ & Y & Z \\
\hline 1. & 6. & 0. & 3.670743 & 0.815299 & 1.907954 \\
\hline 2. & 6. & 0. & 4.364288 & 0.372265 & 2.563884 \\
\hline 3. & 6. & 0. & 4.841848 & $\overline{1.441943}$ & 1.669484 \\
\hline 4. & 6. & 0. & 4.745982 & $\overline{1.382452}$ & 0.326628 \\
\hline 5. & 6. & 0. & 4.070187 & 0.207525 & $\overline{0}-378142$ \\
\hline 6. & 6. & 0. & 4.137415 & 1.109867 & 0.464995 \\
\hline 7. & 6. & 0. & 5.266150 & - & - \\
\hline
\end{tabular}




\begin{tabular}{|c|c|c|c|c|c|}
\hline & & & & 2.480470 & 0.557334 \\
\hline 8. & 6. & 0. & 2.658281 & $\begin{array}{l}- \\
0.624549\end{array}$ & $\overline{0}-740412$ \\
\hline 9. & 6. & 0. & 2.236232 & $\begin{array}{l}- \\
0.874821\end{array}$ & $\begin{array}{l}- \\
1.981096\end{array}$ \\
\hline 10. & 6. & 0. & 0.849158 & $\begin{array}{l}- \\
1.328754\end{array}$ & $\begin{array}{l}- \\
2.365204\end{array}$ \\
\hline 11. & 6. & 0. & 0.845777 & $\begin{array}{l}- \\
2.744626\end{array}$ & $\begin{array}{l}- \\
2.945914\end{array}$ \\
\hline 12. & 8. & 0. & $\overline{-}-040409$ & $\begin{array}{l}- \\
1.321544\end{array}$ & $\begin{array}{l}- \\
1.224852\end{array}$ \\
\hline 13. & 6. & 0. & 5.590877 & 1.628131 & 0.478433 \\
\hline 14. & 6. & 0. & 3.238842 & 2.194326 & $-\overline{0.153664}$ \\
\hline 15. & 8. & 0. & 4.494885 & $\begin{array}{l}- \\
0.447449\end{array}$ & 3.778054 \\
\hline 16. & 1. & 0. & 4.610804 & $\begin{array}{l}- \\
0.026140\end{array}$ & $\begin{array}{l} \\
1.317843\end{array}$ \\
\hline 17. & 6. & 0. & $\begin{array}{l}- \\
1.016555\end{array}$ & $\begin{array}{l}- \\
0.332725\end{array}$ & $\begin{array}{l}- \\
1.218038\end{array}$ \\
\hline 18. & 8. & 0. & 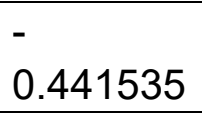 & 0.930844 & $-\overline{0.939830}$ \\
\hline 19. & 6. & 0. & $\begin{array}{l}- \\
1.407936\end{array}$ & 1.996408 & 0.978801 \\
\hline 20. & 6. & 0. & $\begin{array}{l}- \\
2.449813\end{array}$ & 1.772302 & 0.124886 \\
\hline 21. & 6. & 0. & $\begin{array}{l}- \\
3.084179\end{array}$ & 0.382226 & $-\overline{0.029860}$ \\
\hline 22. & 6. & 0. & $\begin{array}{l}- \\
2.013106\end{array}$ & $\begin{array}{l}- \\
0.699876\end{array}$ & $\begin{array}{l}- \\
0.112547\end{array}$ \\
\hline 23. & 8. & 0. & $\begin{array}{l}- \\
2.622127\end{array}$ & $\begin{array}{l}- \\
1.960270\end{array}$ & $\begin{array}{l}- \\
0.414450\end{array}$ \\
\hline 24. & 8. & 0. & $\begin{array}{l}- \\
3.420607 \\
\end{array}$ & 2.790243 & 0.017233 \\
\hline 25. & 8. & 0. & $\begin{array}{l}- \\
3.927801\end{array}$ & 0.211305 & 1.137407 \\
\hline 26. & 16. & 0. & $\begin{array}{l}- \\
5.283464 \\
\end{array}$ & 0.730063 & 1.015976 \\
\hline 27. & 8. & 0. & 6.090647 & $\begin{array}{l}- \\
0.374606\end{array}$ & 2.157084 \\
\hline 28. & 8. & 0. & $\begin{array}{l}- \\
5.772622\end{array}$ & $\begin{array}{l}- \\
0.637328\end{array}$ & $\begin{array}{l}- \\
0.350732\end{array}$ \\
\hline 29. & 8. & 0. & - & - & 1.293199 \\
\hline
\end{tabular}




\begin{tabular}{|c|c|c|c|c|c|}
\hline & & & 4.673423 & 2.183874 & \\
\hline 30. & 6. & 0. & 0.619630 & 3.291796 & 0.820041 \\
\hline 31. & 8. & 0. & 0.337765 & 3.440015 & 1.850339 \\
\hline 32. & 1. & 0. & 3.809351 & 1.686308 & 2.556290 \\
\hline 33. & 1. & 0. & 2.593409 & 0.596652 & 1.914103 \\
\hline 34. & 1. & 0. & 5.307257 & 2.291686 & 2.162898 \\
\hline 35. & 1. & 0. & 5.644242 & 3.325555 & 0.022542 \\
\hline 36. & 1. & 0. & 4.479592 & $\begin{array}{l}- \\
2.840485\end{array}$ & $\begin{array}{l}- \\
1.230578\end{array}$ \\
\hline 37. & 1. & 0. & 6.079042 & $\begin{array}{l}- \\
2.107867\end{array}$ & $\begin{array}{l}- \\
1.194280\end{array}$ \\
\hline 38. & 1. & 0. & 1.954667 & $\begin{array}{l}- \\
0.743936\end{array}$ & 0.079547 \\
\hline 39. & 1. & 0. & 2.922987 & $\begin{array}{l}- \\
0.772788\end{array}$ & $\begin{array}{l}- \\
2.822223\end{array}$ \\
\hline 40. & 1. & 0. & 0.448198 & 0.634223 & 3.117897 \\
\hline 41. & 1. & 0. & 1.526259 & 2.813150 & - 3.799933 \\
\hline 42. & 1. & 0. & 1.175322 & $\begin{array}{l}- \\
3.460162\end{array}$ & 2.187035 \\
\hline 43. & 1. & 0. & $\begin{array}{l}- \\
0.157501\end{array}$ & $\begin{array}{l}- \\
3.021313\end{array}$ & $\begin{array}{l}- \\
3.283749\end{array}$ \\
\hline 44. & 1. & 0. & 6.288399 & 0.893987 & 0.890709 \\
\hline 45. & 1. & 0. & 5.923669 & 1.877419 & 0.535376 \\
\hline 46. & 1. & 0. & 5.665776 & 2.536814 & 1.084737 \\
\hline 47. & 1. & 0. & 2.186597 & 1.897016 & $-\overline{0} 138841$ \\
\hline 48. & 1. & 0. & 3.331814 & 3.132086 & 0.403911 \\
\hline 49. & 1. & 0. & 3.523087 & 2.398284 & $\begin{array}{l}- \\
1.192589\end{array}$ \\
\hline 50. & 1. & 0. & $\begin{array}{l}- \\
1.538519\end{array}$ & $-\overline{0.290646}$ & $\begin{array}{l}- \\
2.192221\end{array}$ \\
\hline 51. & 1. & 0. & $\overline{1.911200}$ & 2.002459 & $\begin{array}{l}- \\
1.957841\end{array}$ \\
\hline 52. & 1. & 0. & 1.936297 & 1.806444 & 1.099125 \\
\hline 53. & 1. & 0. & - & 0.365158 & - \\
\hline
\end{tabular}




\begin{tabular}{|c|c|c|c|c|c|}
\hline & & & 3.713860 & & 0.925355 \\
\hline 54. & 1. & 0. & 1.464691 & 0.753113 & 0.837117 \\
\hline 55. & 1. & 0. & $\begin{array}{l}- \\
1.898957\end{array}$ & $\begin{array}{l}- \\
2.590173\end{array}$ & $\begin{array}{l}- \\
0.557529\end{array}$ \\
\hline 56. & 1. & 0. & $\begin{array}{l}- \\
4.086156\end{array}$ & 2.631672 & 0.702056 \\
\hline 57. & 1. & 0. & $\begin{array}{l}- \\
3.944984\end{array}$ & $\begin{array}{l}- \\
2.333231\end{array}$ & 0.631523 \\
\hline 58. & 1. & 0. & $\begin{array}{l} \\
1.307272\end{array}$ & 4.137744 & $\begin{array}{l} \\
0.882132 \\
\end{array}$ \\
\hline 59. & 1. & 0. & $\overline{-}-152183$ & 3.303055 & 0.177711 \\
\hline 60. & 1. & 0. & 0.881355 & 2.639618 & $\begin{array}{l}- \\
1.835826\end{array}$ \\
\hline & & & & & \\
\hline
\end{tabular}

\begin{tabular}{|l|l|l|l|l|l|}
\hline \multicolumn{2}{|c|}{$3-6 S-4$} & \multicolumn{5}{c|}{$\begin{array}{c}\text { Standard Orientation } \\
\text { (Angstroms) }\end{array}$} \\
\hline $\begin{array}{c}\text { Center } \\
\text { number }\end{array}$ & $\begin{array}{c}\text { Atom } \\
\text { number }\end{array}$ & \multicolumn{1}{|c|}{ Type } & \multicolumn{1}{c|}{ X } & \multicolumn{1}{c|}{ Y } & \multicolumn{1}{c|}{ Z } \\
\hline 1. & 6. & 0. & 2.168838 & 1.386576 & 0.656521 \\
\hline 2. & 6. & 0. & 2.488911 & 1.428723 & - \\
\hline & & & & - & - \\
\hline 3. & 6. & 0. & 3.441868 & 0.448502 & 1.353749 \\
\hline & 6. & 0. & 3.906782 & 0.583120 & 0.615376 \\
\hline 5. & 6. & 0. & 3.433138 & 0.794583 & 0.815294 \\
\hline 6. & 6. & 0. & 3.166700 & 0.585942 & 1.519888 \\
\hline 7. & 6. & 0. & 4.890474 & 1.576119 & 1.164390 \\
\hline 8. & 6. & 0. & 2.235019 & 1.735929 & 0.879638 \\
\hline 9. & 6. & 0. & 1.653621 & 2.394167 & 0.127784 \\
\hline 10. & 6. & 0. & 0.410068 & 3.250768 & 0.006973 \\
\hline 11. & 6. & 0. & 0.714229 & 4.743430 & 0.114990 \\
\hline
\end{tabular}




\begin{tabular}{|c|c|c|c|c|c|}
\hline 12. & 8. & 0. & $\begin{array}{l}- \\
0.486960\end{array}$ & 2.959037 & $\begin{array}{l}- \\
1.112628 \\
\end{array}$ \\
\hline 13. & 6. & 0. & 4.502287 & $\begin{array}{l}- \\
1.348711\end{array}$ & 1.662137 \\
\hline 14. & 6. & 0. & 2.585200 & $-\overline{0.407940}$ & 2.935052 \\
\hline 15. & 8. & 0. & 1.955900 & $\begin{array}{l}- \\
2.250551\end{array}$ & $\begin{array}{l}- \\
1.577509\end{array}$ \\
\hline 16. & 1. & 0. & 4.247730 & 1.280239 & \begin{tabular}{|l|}
1.375631 \\
\end{tabular} \\
\hline 17. & 6. & 0. & $\begin{array}{l} \\
1.456250\end{array}$ & 1.959207 & $\begin{array}{l} \\
0.962904\end{array}$ \\
\hline 18. & 8. & 0. & $\begin{array}{l} \\
2.394876 \\
\end{array}$ & 2.313547 & 0.038934 \\
\hline 19. & 6. & 0. & $\begin{array}{l} \\
3.510195\end{array}$ & 1.413340 & 0.050406 \\
\hline 20. & 6. & 0. & $\begin{array}{l}- \\
3.038316 \\
\end{array}$ & 0.014201 & 0.495745 \\
\hline 21. & 6. & 0. & $\begin{array}{l}- \\
1.886439\end{array}$ & $\begin{array}{l}- \\
0.474427\end{array}$ & $\begin{array}{l} \\
0.380738 \\
\end{array}$ \\
\hline 22. & 6. & 0. & $\begin{array}{l}- \\
0.838189\end{array}$ & 0.597355 & $\begin{array}{l} \\
0.621616 \\
\end{array}$ \\
\hline 23. & 8. & 0. & 0.009550 & 0.143366 & $\begin{array}{l} \\
1.658569 \\
\end{array}$ \\
\hline 24. & 8. & 0. & $\begin{array}{l} \\
4.153692\end{array}$ & $\begin{array}{l}- \\
0.861612\end{array}$ & 0.442975 \\
\hline 25. & 8. & 0. & $\begin{array}{l} \\
1.191981\end{array}$ & $\begin{array}{l} \\
1.543335\end{array}$ & 0.343150 \\
\hline 26. & 16. & 0. & $\begin{array}{l}- \\
1.298360\end{array}$ & $\begin{array}{l}- \\
3.084502\end{array}$ & $\begin{array}{l}- \\
0.185975 \\
\end{array}$ \\
\hline 27. & 8. & 0. & $\begin{array}{l}- \\
0.547271\end{array}$ & $\begin{array}{l}- \\
3.839420\end{array}$ & 0.798517 \\
\hline 28. & 8. & 0. & $\begin{array}{l} \\
2.703585\end{array}$ & $\begin{array}{l} \\
3.361448\end{array}$ & $\begin{array}{l}- \\
0.462580\end{array}$ \\
\hline 29. & 8. & 0. & $\begin{array}{l}- \\
0.554916\end{array}$ & $\begin{array}{l}- \\
3.041250\end{array}$ & $\begin{array}{l} \\
1.582181\end{array}$ \\
\hline 30. & 6. & 0. & $\begin{array}{l}- \\
4.576900\end{array}$ & 1.995483 & 0.985056 \\
\hline 31. & 8. & 0. & $\begin{array}{l}- \\
5.822431\end{array}$ & 1.347956 & 0.842810 \\
\hline 32. & 1. & 0. & 2.065258 & $\begin{array}{l}- \\
2.418294\end{array}$ & 1.010263 \\
\hline 33. & 1. & 0. & 1.167259 & $\begin{array}{l}- \\
0.949937\end{array}$ & 0.737281 \\
\hline
\end{tabular}




\begin{tabular}{|c|c|c|c|c|c|}
\hline 34. & 1. & 0. & 3.738246 & $\begin{array}{l}- \\
0.584052\end{array}$ & $\begin{array}{l}- \\
2.390580\end{array}$ \\
\hline 35. & 1. & 0. & 5.081067 & 1.423088 & $\begin{array}{l}- \\
2.229186 \\
\end{array}$ \\
\hline 36. & 1. & 0. & 4.542142 & 2.603553 & 1.009566 \\
\hline 37. & 1. & 0. & 5.844357 & 1.489310 & $\begin{array}{l}- \\
0.627702\end{array}$ \\
\hline 38. & 1. & 0. & 1.814919 & 1.862966 & 1.876230 \\
\hline 39. & 1. & 0. & 2.058227 & 2.334934 & $\begin{array}{l}- \\
1.138428 \\
\end{array}$ \\
\hline 40. & 1. & 0. & $\begin{array}{l}- \\
0.102227\end{array}$ & 3.049037 & 0.940521 \\
\hline 41. & 1. & 0. & 1.385514 & 5.055195 & 0.690290 \\
\hline 42. & 1. & 0. & 1.192174 & 4.967418 & $\begin{array}{l}- \\
1.073634\end{array}$ \\
\hline 43. & 1. & 0. & $\begin{array}{l}- \\
0.213419\end{array}$ & 5.317641 & $\begin{array}{l}- \\
0.050739\end{array}$ \\
\hline 44. & 1. & 0. & 4.990810 & $\begin{array}{l}- \\
1.527358\end{array}$ & 0.701111 \\
\hline 45. & 1. & 0. & 5.200460 & $\begin{array}{l}- \\
0.791966\end{array}$ & 2.297225 \\
\hline 46. & 1. & 0. & 4.330968 & $\begin{array}{l}- \\
2.321550\end{array}$ & 2.133477 \\
\hline 47. & 1. & 0. & 1.573622 & 0.006342 & 2.928139 \\
\hline 48. & 1. & 0. & 2.528351 & $\begin{array}{l}- \\
1.379024\end{array}$ & 3.436698 \\
\hline 49. & 1. & 0. & 3.220801 & 0.242658 & 3.546838 \\
\hline 50. & 1. & 0. & $\begin{array}{l}- \\
1.951992\end{array}$ & 1.909152 & $\begin{array}{l}- \\
1.944296\end{array}$ \\
\hline 51. & 1. & 0. & $\begin{array}{l}- \\
3.945233 \\
\end{array}$ & 1.338262 & $\begin{array}{l}- \\
0.958604\end{array}$ \\
\hline 52. & 1. & 0. & $\begin{array}{l}- \\
2.656875 \\
\end{array}$ & 0.101281 & 1.525871 \\
\hline 53. & 1. & 0. & 2.266867 & $\begin{array}{l}- \\
0.855314\end{array}$ & $\begin{array}{l}- \\
1.333081\end{array}$ \\
\hline 54. & 1. & 0. & $\begin{array}{l}- \\
0.290290\end{array}$ & 0.719218 & 0.321506 \\
\hline 55. & 1. & 0. & 0.594008 & 0.877024 & $\begin{array}{l}- \\
1.893416\end{array}$ \\
\hline 56. & 1. & 0. & $\begin{array}{l}- \\
3.850273 \\
\end{array}$ & $\begin{array}{l}- \\
1.783906\end{array}$ & 0.384037 \\
\hline 57. & 1. & 0. & 0.427716 & - & \\
\hline
\end{tabular}




\begin{tabular}{|l|l|l|l|l|l|}
\hline & & & & 2.808509 & 1.481604 \\
\hline 58. & 1. & 0. & 4.199893 & 1.961026 & 2.021592 \\
\hline 59. & 1. & 0. & 4.721834 & 3.048165 & 0.724033 \\
\hline 60. & 1. & 0. & 5.653936 & 0.393833 & 0.900383 \\
\hline & & & & & \\
\hline & & & & & \\
\hline
\end{tabular}

\begin{tabular}{|c|c|c|c|c|c|}
\hline \multicolumn{2}{|c|}{$3-6 S-5$} & \multicolumn{4}{|c|}{$\begin{array}{c}\text { Standard Orientation } \\
\text { (Angstroms) }\end{array}$} \\
\hline $\begin{array}{l}\text { Center } \\
\text { number }\end{array}$ & $\begin{array}{l}\text { Atom } \\
\text { number }\end{array}$ & Type & $\mathrm{X}$ & $\mathrm{Y}$ & Z \\
\hline 1. & 6. & 0. & 5.167759 & $\overline{1.598613}$ & 0.438203 \\
\hline 2. & 6. & 0. & 5.986159 & $\begin{array}{l}- \\
0.682602\end{array}$ & $\begin{array}{l}- \\
0.464983\end{array}$ \\
\hline 3. & 6. & 0. & 5.510034 & 0.704499 & $-\overline{0.619099}$ \\
\hline 4. & 6. & 0. & 4.428454 & 1.188634 & 0.024099 \\
\hline 5. & 6. & 0. & 3.566422 & 0.299964 & 0.906027 \\
\hline 6. & 6. & 0. & 4.395562 & 0.867453 & 1.556086 \\
\hline 7. & 6. & 0. & 4.022793 & 2.633118 & $\overline{-}-077143$ \\
\hline 8. & 6. & 0. & 2.328630 & $\overline{-}-196873$ & 0.167893 \\
\hline 9. & 6. & 0. & 2.216508 & $\begin{array}{l}- \\
0.411525\end{array}$ & $\begin{array}{l}- \\
1.145077\end{array}$ \\
\hline 10. & 6. & 0. & 1.002310 & $\begin{array}{l}- \\
0.978934\end{array}$ & $\begin{array}{l}- \\
1.842822\end{array}$ \\
\hline 11. & 6. & 0. & 1.300328 & $\begin{array}{l}- \\
2.335193\end{array}$ & $\begin{array}{l}- \\
2.485050\end{array}$ \\
\hline 12. & 8. & 0. & $\begin{array}{l}- \\
0.109304\end{array}$ & $\begin{array}{l}- \\
1.171243\end{array}$ & $-\overline{0.936115}$ \\
\hline 13. & 6. & 0. & 5.379127 & $\begin{array}{l}- \\
0.279183\end{array}$ & 2.589503 \\
\hline 14. & 6. & 0. & 3.475113 & $\begin{array}{l}- \\
1.862929\end{array}$ & 2.284633 \\
\hline 15. & 8. & 0. & 6.983122 & $\begin{array}{l} \\
1.085013\end{array}$ & $\begin{array}{l}- \\
1.049640\end{array}$ \\
\hline
\end{tabular}




\begin{tabular}{|c|c|c|c|c|c|}
\hline 16. & 1. & 0. & 3.196110 & 0.921052 & 1.734792 \\
\hline 17. & 6. & 0. & $\begin{array}{l}- \\
1.092790\end{array}$ & $\overline{-} .190432$ & $\begin{array}{l}- \\
0.964022\end{array}$ \\
\hline 18. & 8. & 0. & $\begin{array}{l}- \\
0.598019\end{array}$ & 1.011286 & $\begin{array}{l}- \\
0.407380\end{array}$ \\
\hline 19. & 6. & 0. & $\begin{array}{l}- \\
1.547654\end{array}$ & 2.088325 & $\begin{array}{l}- \\
0.449361\end{array}$ \\
\hline 20. & 6. & 0. & $\begin{array}{l}- \\
2.748426\end{array}$ & 1.723098 & 0.429093 \\
\hline 21. & 6. & 0. & $\begin{array}{l}- \\
3.337560\end{array}$ & 0.381029 & $\begin{array}{l}- \\
0.037734\end{array}$ \\
\hline 22. & 6. & 0. & $\begin{array}{l}- \\
2.261654\end{array}$ & $\begin{array}{l}- \\
0.699648\end{array}$ & $\begin{array}{l} \\
0.113398 \\
\end{array}$ \\
\hline 23. & 8. & 0. & $\begin{array}{l}- \\
2.808660\end{array}$ & $\begin{array}{l}- \\
1.883698\end{array}$ & $\begin{array}{l}- \\
0.705110\end{array}$ \\
\hline 24. & 8. & 0. & $\begin{array}{l}- \\
3.697040\end{array}$ & 2.761267 & 0.325386 \\
\hline 25. & 8. & 0. & $\begin{array}{l}- \\
4.356005\end{array}$ & 0.048284 & 0.939655 \\
\hline 26. & 16. & 0. & $\begin{array}{l}- \\
5.672304\end{array}$ & $\begin{array}{l}- \\
0.833066\end{array}$ & 0.458859 \\
\hline 27. & 8. & 0. & $\begin{array}{l}- \\
6.649611 \\
\end{array}$ & $\begin{array}{l}- \\
0.645678\end{array}$ & \begin{tabular}{|l|l}
1.502788 \\
\end{tabular} \\
\hline 28. & 8. & 0. & $\begin{array}{l}- \\
5.940472\end{array}$ & $\begin{array}{l}- \\
0.513723\end{array}$ & \begin{tabular}{|l|}
- \\
0.934487 \\
\end{tabular} \\
\hline 29. & 8. & 0. & $\begin{array}{l}- \\
5.107740\end{array}$ & $\begin{array}{l}- \\
2.324904\end{array}$ & 0.591871 \\
\hline 30. & 6. & 0. & $\begin{array}{l}- \\
0.769062\end{array}$ & 3.316640 & 0.009785 \\
\hline 31. & 8. & 0. & 0.364579 & 3.515077 & \begin{tabular}{|l|}
- \\
0.816584 \\
\end{tabular} \\
\hline 32. & 1. & 0. & 5.841678 & $\begin{array}{l}-358277 \\
\end{array}$ & 0.846688 \\
\hline 33. & 1. & 0. & 4.455767 & $\begin{array}{l}- \\
2.127243\end{array}$ & \begin{tabular}{|l|}
- \\
0.210273 \\
\end{tabular} \\
\hline 34. & 1. & 0. & 6.115703 & 1.340053 & $-\overline{1.260757}$ \\
\hline 35. & 1. & 0. & 4.573957 & 3.159190 & $\begin{array}{l}- \\
0.860158 \\
\end{array}$ \\
\hline 36. & 1. & 0. & 2.950116 & 2.751328 & $\begin{array}{l} \\
0.263968 \\
\end{array}$ \\
\hline 37. & 1. & 0. & 4.221468 & 3.141154 & 0.876223 \\
\hline 38. & 1. & 0. & 1.465493 & - & 0.792111 \\
\hline
\end{tabular}




\begin{tabular}{|c|c|c|c|c|c|}
\hline & & & & 0.408238 & \\
\hline 39. & 1. & 0. & 3.054879 & $\begin{array}{l}- \\
0.204063\end{array}$ & $-\overline{1.809106}$ \\
\hline 40. & 1. & 0. & 0.682427 & $\begin{array}{l} \\
0.278443\end{array}$ & $\begin{array}{l}- \\
2.629426\end{array}$ \\
\hline 41. & 1. & 0. & 2.152992 & $\begin{array}{l}- \\
2.258827\end{array}$ & $\begin{array}{l}- \\
3.165842\end{array}$ \\
\hline 42. & 1. & 0. & 1.544000 & $\begin{array}{l} \\
3.069805 \\
\end{array}$ & $\begin{array}{l}- \\
1.712238\end{array}$ \\
\hline 43. & 1. & 0. & 0.435940 & $\begin{array}{l} \\
2.691091\end{array}$ & $-\overline{3.053316}$ \\
\hline 44. & 1. & 0. & 6.065327 & 0.445573 & 2.143930 \\
\hline 45. & 1. & 0. & 4.837109 & 0.222077 & 3.399150 \\
\hline 46. & 1. & 0. & 5.980940 & $\begin{array}{l} \\
1.076621 \\
\end{array}$ & 3.037722 \\
\hline 47. & 1. & 0. & 2.782451 & $\begin{array}{l}- \\
2.359164\end{array}$ & 1.599021 \\
\hline 48. & 1. & 0. & 4.073031 & $\begin{array}{l}- \\
2.639080\end{array}$ & 2.773958 \\
\hline 49. & 1. & 0. & 2.885111 & $\begin{array}{l} \\
1.361974\end{array}$ & 3.061250 \\
\hline 50. & 1. & 0. & $\begin{array}{l}- \\
1.433608\end{array}$ & $\begin{array}{l}- \\
0.003445\end{array}$ & $\begin{array}{l}- \\
1.999730\end{array}$ \\
\hline 51. & 1. & 0. & $\begin{array}{l} \\
1.887894\end{array}$ & 2.244461 & $\begin{array}{l}- \\
1.484687\end{array}$ \\
\hline 52. & 1. & 0. & $\begin{array}{l} \\
2.396628 \\
\end{array}$ & 1.599469 & 1.465824 \\
\hline 53. & 1. & 0. & $\begin{array}{l} \\
3.814974 \\
\end{array}$ & 0.513060 & $\begin{array}{l} \\
1.014212\end{array}$ \\
\hline 54. & 1. & 0. & $\begin{array}{l} \\
1.880466 \\
\end{array}$ & $\begin{array}{l} \\
0.912040\end{array}$ & 0.894067 \\
\hline 55. & 1. & 0. & $\begin{array}{l}- \\
2.071733 \\
\end{array}$ & $\begin{array}{l} \\
2.502807\end{array}$ & $\begin{array}{l}- \\
0.822569\end{array}$ \\
\hline 56. & 1. & 0. & $\begin{array}{l}- \\
4.464716 \\
\end{array}$ & 2.509331 & 0.858594 \\
\hline 57. & 1. & 0. & $\begin{array}{l} \\
4.282107 \\
\end{array}$ & $\begin{array}{l}- \\
2.383303 \\
\end{array}$ & 0.038523 \\
\hline 58. & 1. & 0. & \begin{tabular}{|l|}
- \\
1.401432 \\
\end{tabular} & 4.203733 & $\begin{array}{l}- \\
0.065514\end{array}$ \\
\hline 59. & 1. & 0. & $\begin{array}{l}- \\
0.485697\end{array}$ & 3.183494 & 1.066300 \\
\hline 60. & 1. & 0. & 0.782486 & 2.644722 & 0.900911 \\
\hline
\end{tabular}






